\title{
HALTBUURTON
}

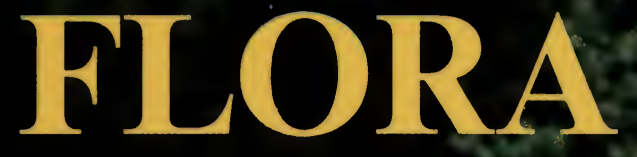

\section{AN ANNOTATED LIST OF THE VASCULAR PLANTS OF THE COUNTY OF HALIBURTON, ONTARIO}
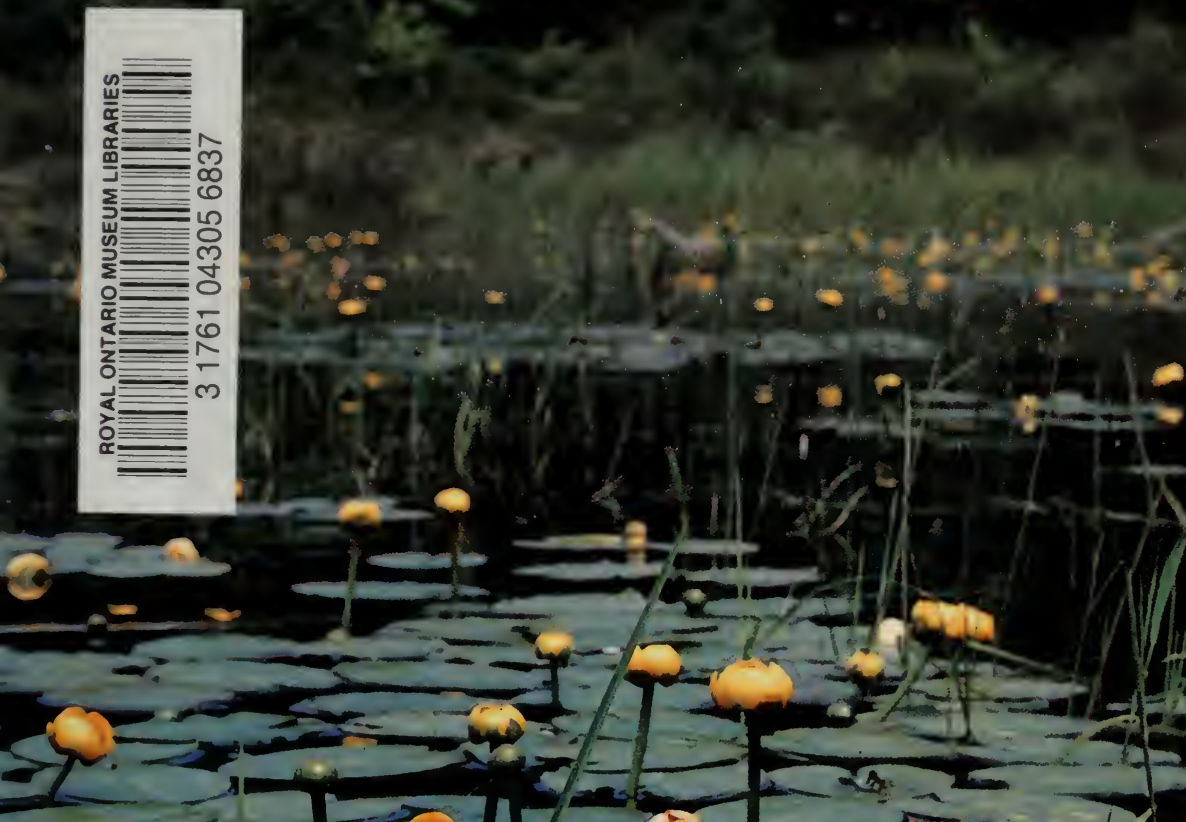

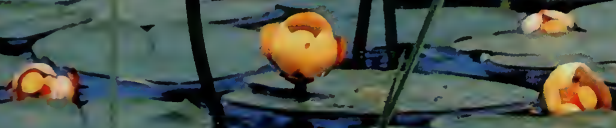
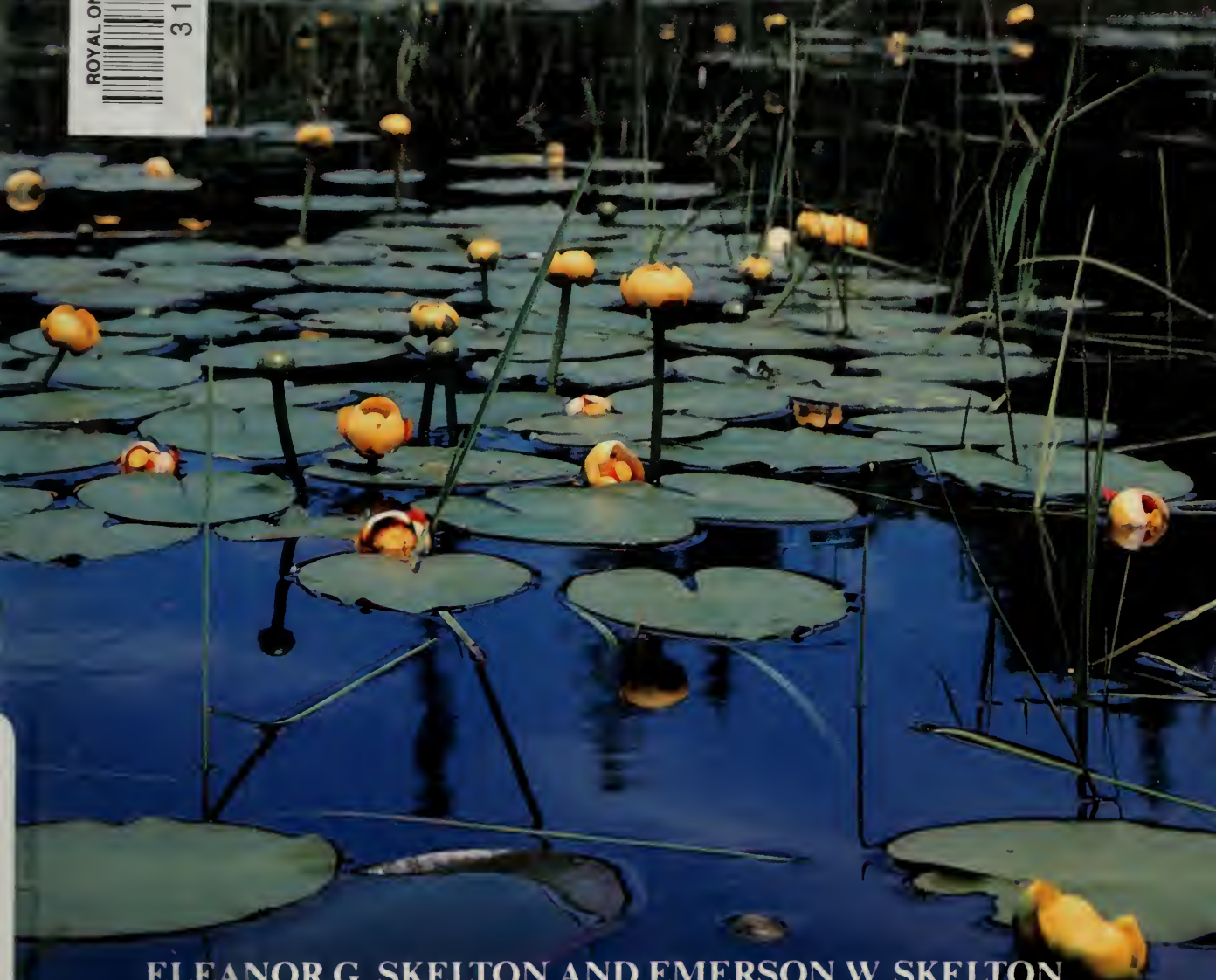


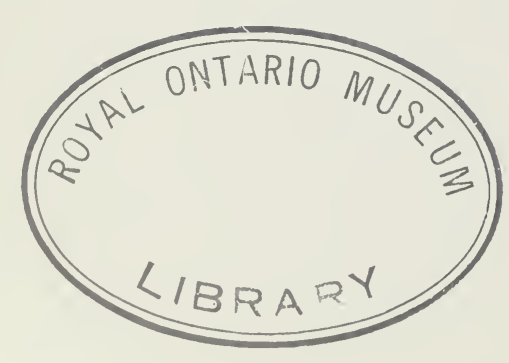




\section{HALIBURTON FLORA}

AN ANNOTATED LIST OF THE VASCULAR PLANTS OF THE COUNTY OF HALIBURTON, ONTARIO

ELEANOR G. SKELTON AND

EMERSON W. SKELTON

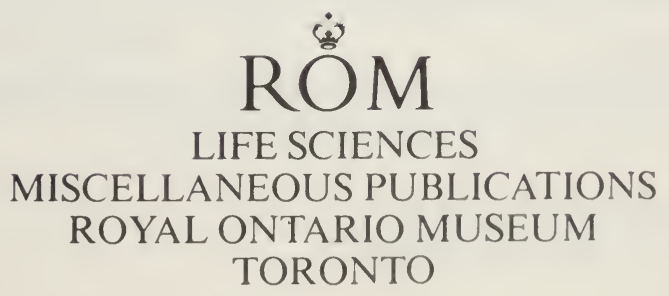




\section{ROYAL ONTARIO MUSEUM \\ PUBLICATIONS IN LIFE SCIENCES}

The Royal Ontario Museum publishes three series in the Life Sciences: CONTRIBUTIONS: a numbered series of original scientific publications.

OCCASIONAL PAPERS: a numbered series of original scientific publications, primarily short and of taxonomic significance.

MISCELLANEOUS PUBLICATIONS: an unnumbered series on a variety of subjects.

All manuscripts considered for publication are subject to the scrutiny and editorial policies of the Life Sciences Editorial Board, and to independent refereeing by two or more persons, other than Museum staff, who are authorities in the particular field involved.

\section{LIFE SCIENCES EDITORIAL BOARD}

Senior editor: J. H. McAndrews

Editor: M. D. Engstrom

Manuscript editor: C. S. Churcher

Editor: G. B. Wiggins

Production editor: J. E. Hawken

External editor: C. S. Churcher

The Royal Ontario Museum is an agency of the Ontario Ministry of Culture and Communications.

\section{Canadian Cataloguing in Publication Data}

Skelton, Eleanor G., 1908-1989

Haliburton Flora : an annotated list of the vascular plants of the county of Haliburton, Ontario

(Life Sciences miscellaneous publications, ISSN 0082-5093) Includes bibliographical references.

ISBN 0-88854-353-0

1. Botany - Ontario - Haliburton (County).

I. Skelton, Emerson W., 1907- ～. II. Royal Ontario

Museum. III. Title. IV. Series.

QK203.05S57 $1990 \quad 581.971361 \quad$ C $90-093701-7$
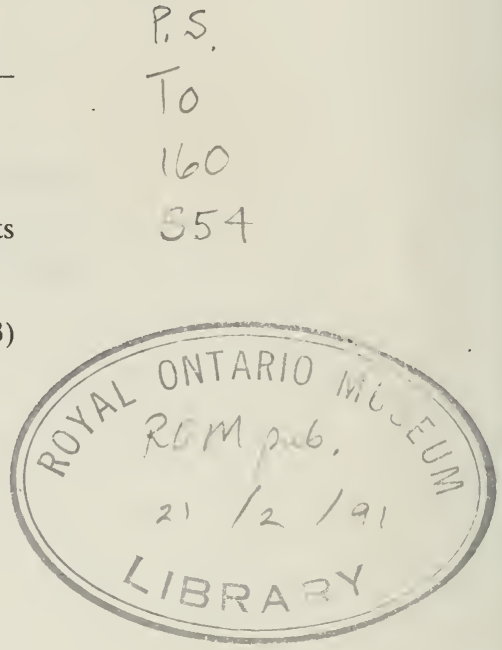

Publication date: 1 February 1991

ISBN 0-88854-353-0

ISSN 0082-5093

(C) Royal Ontario Museum, 1991

100 Queen's Park, Toronto, Canada M5S 2C6

Typesetting by Trigraph Inc.

Printed and bound in Hong Kong by C \& C Offset Printing Company Limited

Colour separation by Goody Color Separation (Scanner) Limited

Cover: Yellow water-lilies, Nuphar variegata,

Haliburton, 1977 (Photo: Emerson W. Skelton) 


\section{CONTENTS}

Foreword 5

Introduction 9

Features of Haliburton Significant to the Flora 9

Topography, Drainage, Roads 9

Rocks and Glacial Deposits 10

Soils 14

Climate $\quad 15$

Forests $\quad 15$

Wetlands $\quad 16$

Open-water Habitats 19

Other Habitats 19

Methods 20

Collecting and Collections $\quad 20$

Territory Covered 20

Identification and Nomenclature $\quad 20$

Voucher Specimens 21

Abundance Ratings 21

Acronyms, Abbreviations, and Symbols 22

Herbarium Acronyms 22

Township Abbreviations 22

Other Abbreviations and Symbols 22

Maps Used in Establishing Locations 23

Annotated List of the Vascular Plants of the County of Haliburton 25

Fern Allies 25

Ferns 27

Conifers 31

Flowering Plants

32

Monocotyledons
Dicotyledons 61

Acknowledgements $\quad 121$

Literature Cited 123

Additional Publications Consulted in Identification

Index to Common Names

127

Index to Scientific Names 


\section{LIST OF FIGURES}

Fig. 1 Location of the county of Haliburton in southern Ontario, and township abbreviations used in the Annotated List 8

Fig. 2 Main highways and larger lakes of the county of Haliburton 11

Fig. 3 Postglacial topography of the county of Haliburton

13 


\section{FOREWORD}

It was with surprise and sorrow that I learned of the passing of Mrs. Eleanor Skelton on 1 October 1989. My memories of Eleanor will be of an extraordinary woman, an impressive botanist, a devoted wife, and a good friend. Born and raised in Toronto, she undoubtedly developed a love of nature and the outdoors during her childhood and youth when she spent her summers at the family cottage on Gull Lake in Haliburton. She pursued her education through the university level and graduated from the University of Toronto with a General Arts degree with emphasis on biology.

Eleanor was ready to accept any challenge. Having decided that biology, especially the study of fish, sparked her interest, Eleanor made inroads into this male-dominated field by gaining employment with the Ontario Fisheries Research Lab from 1929 to 1932. In 1931 she deposited her first plant collection in the herbarium at the University of Toronto (TRT).

It is difficult to talk about Eleanor without mentioning her husband Emerson. They met while they were students at the university and were married in 1931. Their mutual interest in natural history, and in fact in all facets of science, and their enjoyment of the outdoors continued to be important throughout their married life. During the years of Emerson's career as a chemical engineer he became an accomplished photographer of wildflowers.

Emerson retired in 1975 and the couple embarked on a special project. I first met Eleanor and Emerson in 1976 when they were auditing a course in plant taxonomy at the University of Toronto Botany Department and were bringing in plant specimens to identify from their summer holiday in Haliburton. With the enthusiastic support of Dale Hoy, at the time curatorial assistant at TRT, they began their project of documenting the plants of the county of Haliburton. For eight years, from 1976 to 1983, the Skeltons rented a cottage in Haliburton, sometimes departing from the city as early as April and returning to Toronto at Thanksgiving. They conducted field excursions, collecting and carefully pressing plant specimens throughout the growing seasons. During the winter they identified and painstakingly mounted the collections, providing TRT with some of that herbarium's finest specimens. They would mark particular trees or shrubs-in order to collect flowering, fruiting, and mature leaf material from the same individual-and mount all the stages on a single sheet, sometimes even including a close-up photograph of the flower or the habitat. In all they donated more than 2100 herbarium sheets representing more than 930 taxa to the TRT. The neatness and completeness of their specimens are outstanding. The collections are a real asset to the herbarium and are often consulted by artists and researchers; this shows that 
nonprofessional or "amateur" botanists can make very worthwhile contributions to science.

Emerson became the grass and sedge expert of the team, while Eleanor was the aquatics specialist. Aquatic plant communities always commanded a very special place in Eleanor's heart, and she spent innumerable hours in the canoe with Emerson and investigated the submerged, floating, and emergent vegetation of the many rivers and lakes in Haliburton. She made some outstanding discoveries, such as locating a colony of the provincially rare bladderwort Utricularia geminiscapa and an especially large colony of another bladderwort $U$. gibba. Her interest in the effects of acidic precipitation on the vegetation of Haliburton-in particular on the aquatic systems-led her to confer with the Ontario Ministry of Environment staff at Dorset, Ontario, and eventually to contribute lists of plants present at specific lakes in the region to the Acidic Precipitation in Ontario Study.

During the years the Skeltons spent collecting their specimens from Haliburton, Emerson pursued another project. He was involved in annotating and recording specimens of the James H. White Collection, which had been acquired from the University of Toronto Faculty of Forestry. These specimens, from the early 1900's, were donated to TRT in 1976 when their historical significance became apparent.

Once the Skeltons' own collections from Haliburton were completed to their satisfaction, from 1983 to 1986 they took on the mammoth task of writing up their findings and incorporating geologic, topographic, and soil information. This phase also included checking the herbaria in Ottawa and in the Algonquin Park Museum for additional Haliburton records. Their intention was to produce a scientifically valuable work that would be straightforward enough to be used by naturalists. Because of their contributions to the herbarium, the Skeltons became field associates in the Department of Botany of the Royal Ontario Museum in early 1987.

During the final writing stages, Emerson's health began failing, leaving the onus to fall on Eleanor. She rose to the occasion and completed the manuscript, which was then submitted to the Museum for publication. The review process, both internal and external, resulted in further changes that Eleanor ably dealt with right up until the summer of 1989, with help from the Museum's Department of Botany.

In the fall of 1988 the Skeltons had moved to Owen Sound. In the following spring they began exploring their new surroundings and, when I visited that August, even sent specimens back with me to the herbarium. During my visit, Eleanor joined me on a brief trip to collect fruiting specimens of Crataegus douglasii south of Wiarton. Although obviously in pain, she 
showed undaunted interest as she searched the roadsides for this unusual black-fruited hawthorn.

It would have been very satisfying for Eleanor to have been able to browse through Haliburton Flora. Even though this cannot be so, her work in print will be a fitting tribute to this remarkable person and to her husband, both of whom I am very proud to have known. Their retirement project has proved to be a very valuable scientific contribution.

\author{
Sheila McKay-Kuja \\ Department of Botany \\ University of Toronto \\ December 1989
}




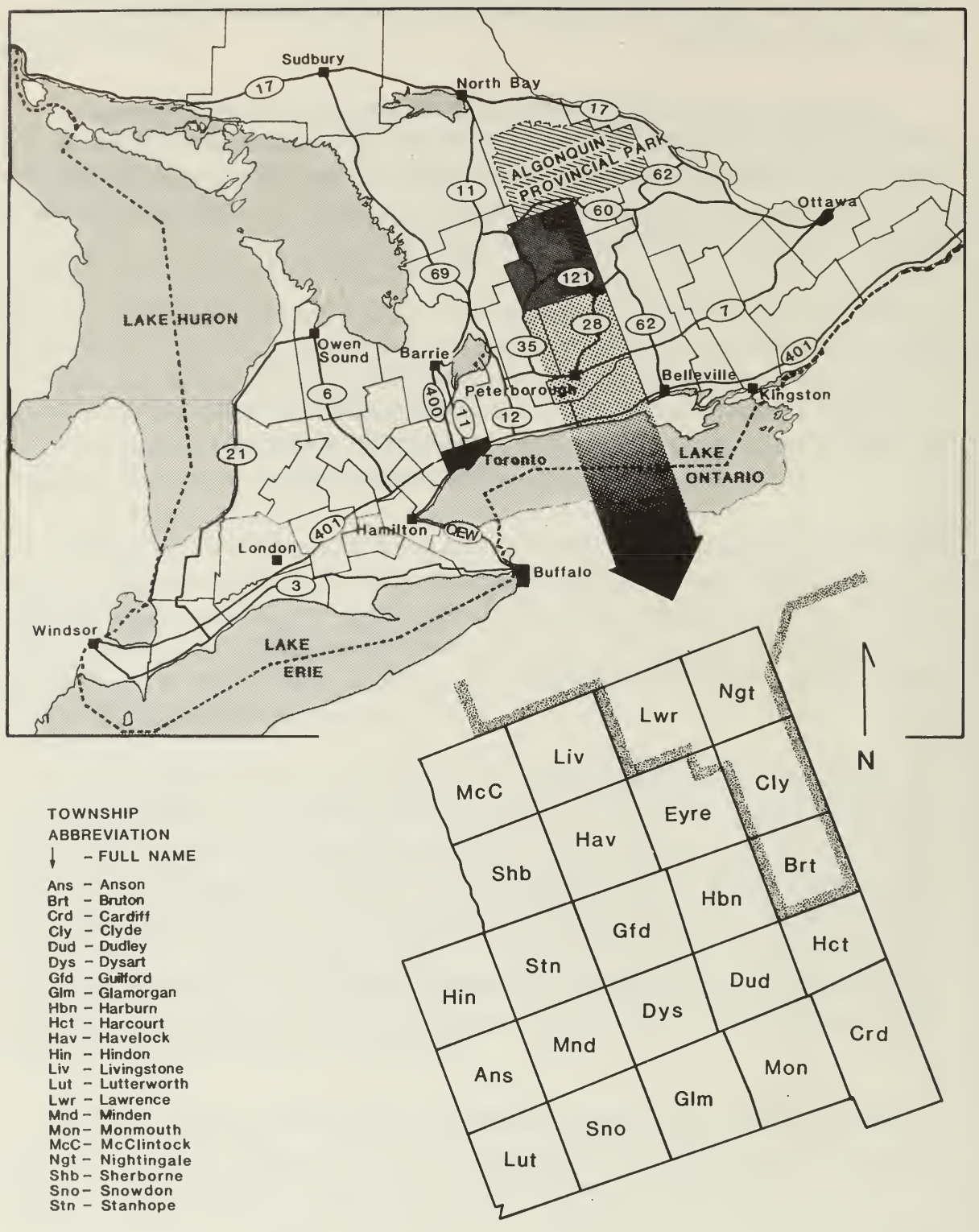

FIG. 1. Location of the county of Haliburton in southern Ontario. Township abbreviations on Figs. 1 and 2 and throughout the annotated list: Ans, Anson; Brt, Bruton; Cly, Clyde; Crd, Cardiff; Dud, Dudley; Dys, Dysart; Gfd, Guilford; Glm, Glamorgan; Hav, Havelock; Hbn, Harburn; Hct, Harcourt; Hin, Hindon; Liv, Livingstone; Lut, Lutterworth; Lwr, Lawrence; McC, McClintock; Mnd, Minden; Mon, Monmouth; Ngt, Nightingale; Shb, Sherborne; Sno, Snowdon; Stn, Stanhope. [Map by Celia Godkin] 


\section{INTRODUCTION}

The county of Haliburton, an area of 452282 hectares, is situated in the heart of southern Ontario's vacationland (Fig. 1). Its more than six hundred lakes, resorts, canoe routes, and wilderness areas attract numerous visitors, cottagers, naturalists, and scientists, and an increasing number of retirees. In the fall tourists pour into the county to enjoy the spectacular display of autumn colours. Even within towns, the lushness of the trees and the colours and configurations of the rock formations provide vistas of scenic beauty.

This largely wooded landscape is, however, occasionally interrupted by open areas and, in its southern parts, by a few viable farms. Often the open areas represent the disappointed hopes of early settlers, or more recent projects of man such as hydro-line cuts, borders of highways, occasional waste dumps, and railway lines now abandoned.

A cumulative list of flora found in the county of Haliburton has not previously been produced, and there are very few records to suggest that early botanists passed this way. Our intention is to bring together the results of our eight years of explorations in the county of Haliburton, on land and on water, and other collectors' records that we found and examined in Ontario herbaria.

The plan to produce a checklist of the vascular plants of the county of Haliburton originated with Dale Hoy (formerly Dale Leadbeater), at the time a curatorial assistant in the herbarium of the Department of Botany, University of Toronto (TRT) and a summer resident of Harcourt Township. Most of the specimens on the list from that township, and some from neighbouring areas, were collected and identified by Dale. We joined her in the project in 1976, and upon her retirement from TRT she produced a preliminary list of our combined collections. The continuation of the project has been carried on with her encouragement.

Undoubtedly there are more vascular plant species to be found in the county of Haliburton than appear here. We hope that this list of 922 named plants, which includes 110 families and 899 species, will be useful to botanists, environmentalists, and naturalists, both professional and nonprofessional.

\section{FEATURES OF HALIBURTON SIGNIFICANT TO THE FLORA}

\section{TOPOGRAPHY, DRAINAGE, ROADS}

The overall contour of Haliburton forms part of the Algonquin dome with the top of the dome just north of the northern boundary of the county, within Algonquin Park. Manitou Mountain, in Lawrence Township and with an ele- 
vation of $564 \mathrm{~m}$, is the highest peak in the county of Haliburton, while the highest lake surface is $482 \mathrm{~m}$ at nearby McGarvey Lake. From this high area the land slopes downwards to east, west, and south, reaching a level of $270 \mathrm{~m}$ at Gull Lake in Lutterworth Township. The terrain throughout is characterized by rugged ridges, clefts, valleys, small pockets or stretches of wetlands, occasional sand or mud flats, a multitude of streams, ponds, and more than 600 lakes. The high rock ridges, slopes, and lowlands provide a variety of habitats for the trees, shrubs, and herbaceous plants that appear on the list.

Eight rivers provide drainage in the county of Haliburton. The Hollow River, originating in Lawrence Township, supplies water to Lake Kawagama, which drains into the south Muskoka River via Lake of Bays. The flow continues through Muskoka Lake and the Moon and Musquash rivers to Georgian Bay. The York River originates in northern Harburn Township and flows east and south before it leaves the county to join the Madawaska River flowing into the Ottawa River system. The Drag, Burnt, Irondale, Crowe, Gull, and Kennisis rivers gather their waters from run-off, bog and swamp seepage, creeks, and chains of lakes before eventually joining the Trent River system, which flows into Lake Ontario.

Much of the water flowing into the Trent-Severn Waterway is controlled by dams in the upper reaches such as Haliburton, in order to maintain a navigable water level farther south. The effect on plant life in Haliburton is quite noticeable, particularly in dry seasons when shorelines are exposed. For example, hedge-hyssop (Gratiola neglecta), a shoreline plant, may increase greatly in numbers because its seeds can withstand long periods of submersion and germinate when exposed. Some species cannot withstand the exposure and decrease or disappear. In unusually wet seasons when the water is held back, water lobelia (Lobelia dortmanna) and lance-leaved violet (Viola lanceolata) have been seen blooming below the surface of the water, while arrowhead (Sagittaria spp.), rooted on the bottom, will grow unusually long stems to reach the surface and will produce few or no flowers.

The main roads through the county are Hwy. 35, entering Lutterworth Township at the southern boundary and running north through Dorset, in Sherborne Township, and Hwy. 121, running east-west through the southern townships (Fig. 2). Many secondary paved roads link popular resort areas and communities, and gravel roads lead into some of the more remote areas. Many old logging roads through "bush country" remain and serve as convenient and rewarding paths for field botanists.

Roadsides usually provide favourable growing conditions for numerous plants because of their openness to sunlight and, in many locations, because of the extra water supply present in damp to wet ditches along their routes.

\section{ROCKS AND GLACIAL DEPOSITS}

The rocks of Haliburton are of Precambrian origin, lying within the Precambrian Shield, and are predominantly granitic. Folding, faulting, warping, volcanism, and metamorphism, along with erosion and sedimentation, pro- 


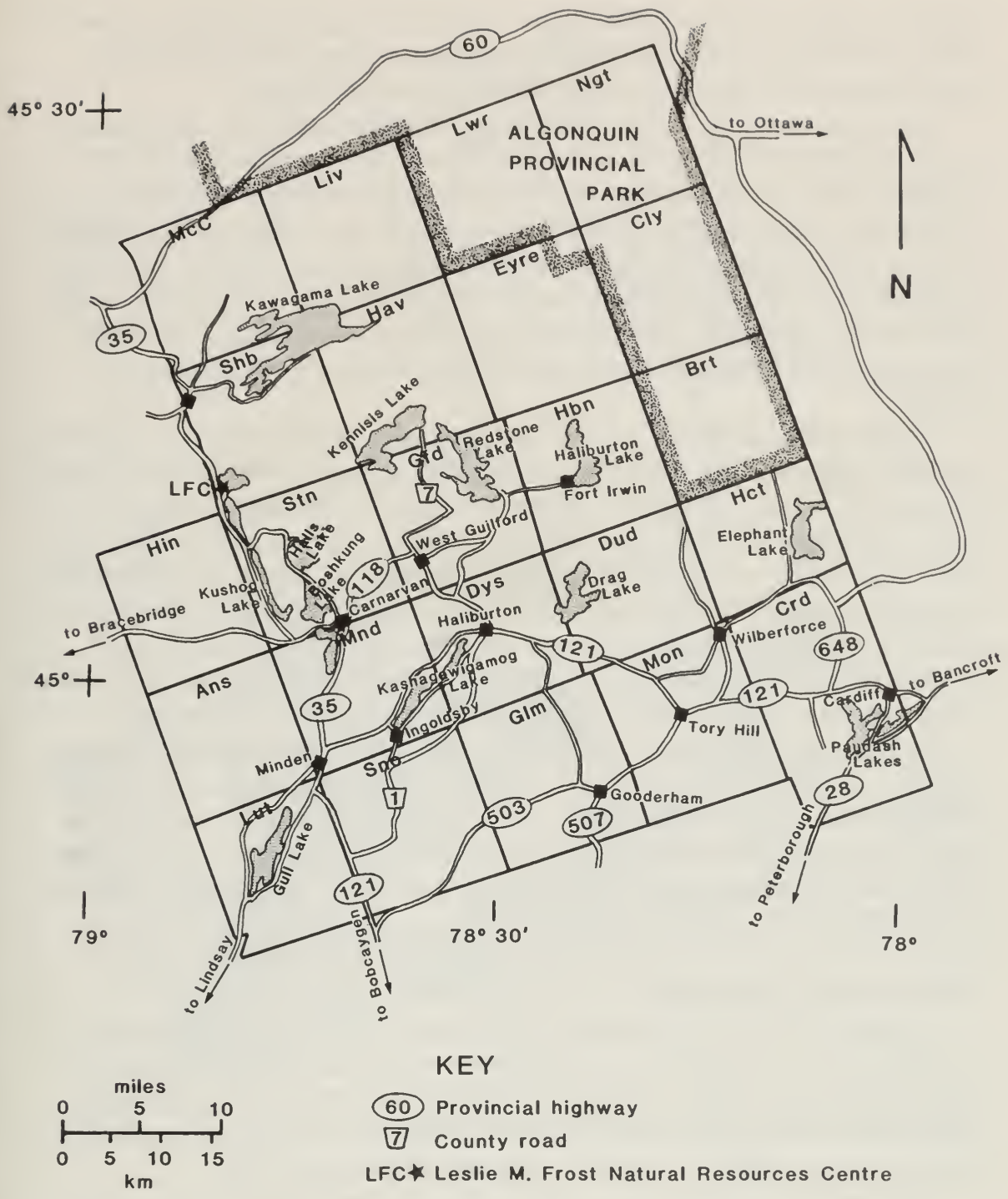

FIG. 2. Main highways and larger lakes of the county of Haliburton. See Fig. 1 caption for list of township abbreviations. [Map by Celia Godkin] 
duced a rugged landscape and a large variety of rocks and minerals. Many of these can be seen in colourful displays of greys, whites, blacks, and pinks along shores and road cuts in horizontal or contorted bands.

The most significant of the Precambrian metamorphosed sedimentary rocks are the Grenville limestones of the Grenville Province described by Douglas (1970). These occur mostly in strips in the central and southern parts of the county (Fig. 3). The Haliburton limestones are mostly coarse grained and impure although pure calcium limestone (calcium carbonate) has occasionally been found, for example, in the Minden-Miner's Bay-Buller area (Lutterworth Township) and in a few locations in Glamorgan Township. Deposits of dolomite and other limestones containing magnesium are common.

Limestones can greatly benefit plant and animal life in the waters that lie over them through their capacity to neutralize acids. It has been concluded by the ongoing research of APIOS (Acidic Precipitation in Ontario Study) of the Ontario Ministry of the Environment that lakes on limestone are better protected against acid precipitation (APIOS, 1981). Of the Haliburton lakes that are shown in the APIOS report to be of lowest sensitivity to acid precipitation, five had been among our favoured collecting sites: Barnum, Duck (Little Elsie), Eels (northeast arm), Paudash, and Marigold lakes. They were found to produce an unusual variety and abundance of aquatic plants.

An association between plant distribution and rock types seemed apparent. It was noted in collecting that a number of species known to have a definite preference for calcareous sites were found in limestone areas either on rock ledges or on thin till over calcareous bedrock. Examples of such plants include the following: cotton-grass (Eriophorum viridi-carinatum), narrowleaved spleenwort (Athyrium pycnocarpon), sedges (Carex aurea, $C$. diandra, C. eburnea, C.garberi, and C. granularis), and white adder'smouth (Malaxis monophylla var. brachypoda).

In preglacial times, rock scarps (cliffs) were created by faulting, that is, the displacement of a rock mass by fracturing during which one side of the split rises above the other. The faults of Haliburton are not of great height compared to those of other regions, but a number have been mapped (Fig. 3) and they clearly affect the courses of streams (Chapman, 1975).

Much of the modern topography of the area was shaped by the Late Wisconsin ice sheet of the Pleistocene epoch, the last glaciation to cover the region, which retreated about 12000 years ago. The advances and retreats of this immense mass of scouring ice rounded off ridges and deposited erratic boulders and till soils consisting of sand, gravel, silt, and sometimes clay. The drift deposits that it laid down (till plains and sand plains) vary in depth according to the contours of the land beneath and the conditions under which the ice melted. Recognizable today are elliptical-based mounds of till called drumlins (Fig. 3) and some drumlinoid rolling hills. A small intermittent esker (a ridge of sand and gravel) extending about $16 \mathrm{~km}$ east and west of Gooderham was reported by Chapman (1975). 


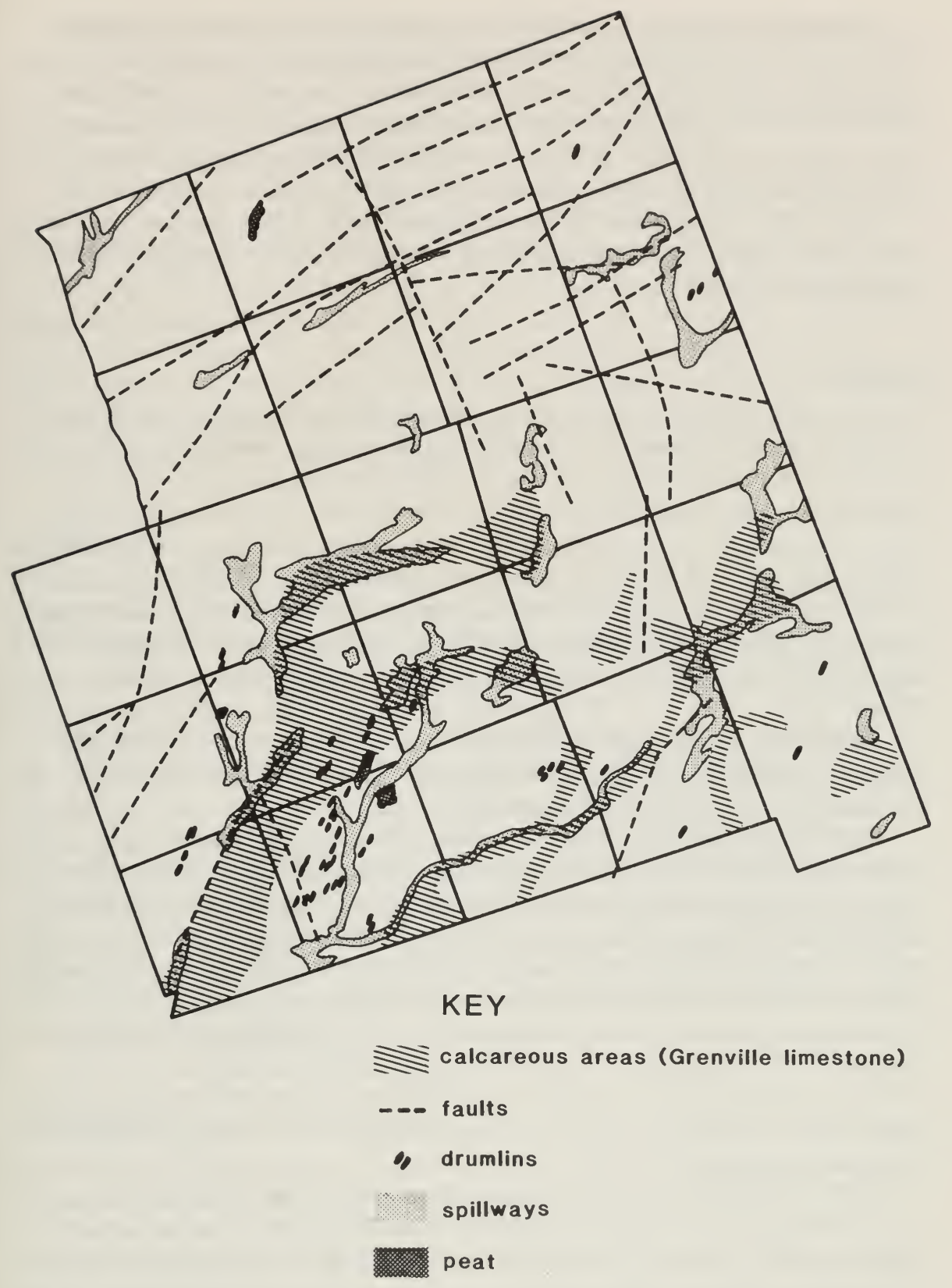

FIG. 3. Postglacial topography of the county of Haliburton (to same scale as Fig. 2). [Map by Celia Godkin] 
During glacial recessions, meltwater sometimes formed broad spillways (Fig. 3) that became filled with sandy outwash through which present river systems flow. Some old spillways contain lakes: for example, the Gull River system of several central and southern townships, and the Irondale-Burnt River system in southern Haliburton. Haliburton, Drag, Gull, Kashagawigamog, Maple, and a number of other lakes also lie in part in old spillways.

For further information on the geology of the area, the reader is referred to the texts used to prepare this account: Chapman (1975), Douglas (1970), and Goudge (1938).

\section{SOILS}

The soils of the county of Haliburton were originally laid down during the last glaciation, the essential materials being ground-up granitic rock of the Precambrian Shield. Subsequent soil development has been advanced by a number of physical and biological processes: erosion, weathering, drainage, recent sand deposition by rivers and lakes, leaching, climate, and interaction of rock particles with living and decayed organic matter. Soil scientists classify soil types according to composition and profile. Four predominant types found in Haliburton are podsols on uplands with deeper soil, brunisols on uplands with shallower soil, regosols on bedrock, and gleisols in poorly drained sites.

The soils are acid ( $\mathrm{pH} 6$ or less) and are relatively low in available phosphorus; potassium levels can be adequate. Limited nitrification occurs although it is more likely where limestone is present.

Bedrock outcrops are often bare of vegetation or may support only lichens and mosses. Frequently they also contain pockets of sandy soil that support small plant species such as bunchberry (Cornus canadensis), early saxifrage (Saxifraga virginiensis), and mossy stonecrop (Sedum acre). In larger rock depressions where a varying amount of topsoil has accumulated, a greater variety of woodland, shoreline, or roadside species may be found depending on location. In some depressions on rock outcrops, coniferous trees send down deep roots in rock fissures for additional sustenance. They provide a certain amount of shade for twinflower (Linnaea borealis) and partridge-berry (Mitchella repens), and other plants of the woods that may be found in these sites.

Upland soils are typically less than $50 \mathrm{~cm}$ deep with a variable thickness of surface organic layers. They support a considerable list of herbaceous plants, shrubs, and trees. The uplands are usually deficient in moisture in midsummer although in the limited areas of drumlins and rolling hills, where the soil is deeper, more moisture is retained. On the lower slopes and in the valleys, soils are moist and contain more humus. Here, the deciduous forests keep adding to the blanket of dead leaves, thereby deterring evaporation and providing more material for the organic layer. Here also, temporary streams last longer into the growing season and ponds are frequent. 
Wetlands are described elsewhere, but it should be mentioned here that their soils are peculiar to areas of impeded drainage and higher groundwater levels. In bogs, muck or peat may form a layer of up to 40 or $50 \mathrm{~cm}$ that is underlain by a layer of mixed mineral and humic materials, and below this a sticky unstructured material (gleisol).

The texture of materials lying in the substrata between topsoils and bedrock is coarse and sandy except in clay areas. Sand also appears at the surface in the flat terrain of glacial, fluvial, or lacustrine deposits at a number of sites and supports scanty or patchy vegetation or none at all. On dry sand flats, species of mosses, grasses, and other plants adaptable to open dry habitats are found: for example, field cress (Lepidium campestre), common plantain (Plantago major), and sheep-sorrel (Rumex acetosella). Among the plants found on wet sand flats are creeping spearwort (Ranunculus reptans) and species of sedges (Carex), spike-rushes (Eleocharis), and rushes (Juncus).

\section{CLIMATE}

The county of Haliburton's popularity as a vacationland, in both winter and summer, is partly a result of its rather congenial climate. Though temperatures have been recorded as high as $40^{\circ} \mathrm{C}$ in July or as low as $-45.5^{\circ} \mathrm{C}$ in January, the climate tends to be moderate and comfortable, lending itself not only to recreation but also to healthy plant growth.

The county lies in two climactic regions (Brown, McKay, and Chapman, 1974). The southern portion of the county belongs in the Haliburton Slopes region, while the upper third is in the Algonquin Park region. The climactic values are essentially the same, though the values for the Algonquin Park region, which is of slightly higher elevation, tend to be $1^{\circ} \mathrm{F}$ cooler than those for the Haliburton Slopes. The mean daily maximum temperature for the region is $-7^{\circ} \mathrm{C}$ in January and $26^{\circ} \mathrm{C}$ in July. The mean date for the last frost of the spring is 25 May, and the mean date of the first frost is 17 September. The county receives a mean annual precipitation of 70 to $100 \mathrm{~cm}$ and a mean annual snowfall of $200 \mathrm{~cm}$.

\section{FORESTS}

The county of Haliburton is located within the Great Lakes-Saint Lawrence Forest region (Rowe, 1972), which lies between boreal forest to the north and deciduous forest to the south. Forests within the county are composed predominately of mixed coniferous and deciduous tree communities, which vary in composition according to the topography of the land (e.g., ridges, valleys, slopes, or wetlands) and the tolerances of species for particular soil and moisture conditions.

The most common and widely distributed tree in Haliburton is the sugar maple (Acer saccharum). It frequently occurs in almost pure stands, but most often it shares mixed woods with a lesser number of other species, which may 
include white ash (Fraxinus americana), basswood (Tilia americana), American beech (Fagus grandifolia), yellow birch (Betula alleghaniensis), ironwood (Ostrya virginiana), balsam-fir (Abies balsamea), eastern hemlock (Tsuga canadensis), and white pine (Pinus strobus).

By late summer, the forest floor in a mature sugar maple forest is usually devoid of undergrowth except where gaps in the canopy let in light that allows shrubs and saplings to grow. In spring, in these dense forests, early flowers bloom before the trees are in full leaf. In species that store their nourishment underground in bulbs or corms-such as Dutchman's breeches (Dicentra cucullaria), squirrel-corn (D. canadensis), wild leek (Allium tricoccum), and spring-beauty (Claytonia caroliniana)-the leaves disappear in a few weeks. Several woodland species in semishaded locations noticeably increase their leaf sizes to gather more light as the season progresses.

In exploring the forests, the hiker can occasionally come upon relics of the era of the great white pine forests, which brought fame and fortune to the lumber barons of the district after 1850 . These relics, which are mostly old rotting stumps or logs, are often nearly hidden by more recent growth but are of immense girth.

When early settlers found that farming was unprofitable, they turned to the abundant tall white pine, and the men of the district spent the winters in the numerous logging camps north of the present village of Haliburton. The camps were situated wherever access to streams and river banks was possible so that in summer the logs could be floated down in drives through chains of rivers and lakes to mills and markets farther south. "When pine was king both saw-logs and squared-timbers were prepared for market in Quebec or the U.S.A. The finest [white] pine of all became masts for the Queen's ships and Haliburton timbers carried canvas sails to every port on the globe" (Reynolds, 1968). Reynolds also states that "the virgin pine was consumed in less than half a century." This refers to a period over the late nineteenth and early twentieth centuries ending with the last log drive down the Gull River system in 1929. Other trees valuable for lumber, which were also depleted along with white pine, were yellow birch, red pine (Pinus resinosa), and hemlock.

Lumbering in Haliburton goes on today, aided by mechanized equipment, trucks, and roads, under a management plan of the Ministry of Natural Resources. The days of the winter lumber camps, heavy manual labour with axe and saw, and the river driver, are now part of the local folklore.

\section{WETLANDS}

The importance of wetlands has been well explained by scientists and others. Owing to the topography of the land, with its many depressions and valleys, and to the plentiful supply of rainfall, numerous wetlands occur in the county of Haliburton. Melting snow, rainfall, and groundwater supply these wetlands, which release the water slowly throughout the season, thereby provid- 
ing habitats for aquatic and semiaquatic plants as well as breeding and feeding grounds for birds and mammals. The preservation of the wetlands in Haliburton is aided by two contributing factors. The fact that the soil is mostly thin and of poor quality for farming has meant that little draining has been done for this purpose. Also, an adequate beaver population creates new wetland habitats by damming streams and flooding new territory. Beaver activity tends to counter-balance the long-term encroachment of vegetation whereby plant succession gradually converts wet areas into dry land. In some instances, flooding has also been caused by the building of roads across streams or wet seepage areas.

Many areas contain more than one wetland type, and neighbouring communities often merge into one another. Based largely on definitions in Jeglum, Boissonneau, and Haavisto (1974) and Pringle (1980), the more commonly encountered types of wetland in Haliburton are briefly described here, with a few representative species given for each.

Bogs. These areas contain organic soils and layers of sphagnum or other peat in the substratum, with acid-loving plants above. Sphagnum spp. form the dominant ground cover. Bogs may be sparsely or thickly treed with black spruce (Picea mariana) and tamarack (Larix laricina), or open with large or small colonies of such low shrubs as sweet gale (Myrica gale) and leatherleaf (Chamaedaphne calyculata). Bogs very frequently occur around the edges of small lakes and ponds where they extend into the lake in large spongy mats and small floating islands. Typical species found in bogs are bog-laurel (Kalmia polifolia), bog-rosemary (Andromeda glaucophylla), three-leaved Solomon's-seal (Smilacina trifolia), cranberries (Vaccinium macrocarpon and $V$. oxycoccus), buckbean (Menyanthes trifoliata), grass-pink (Calopogon tuberosus), rose-pogonia (Pogonia ophioglossoides), pitcher-plant (Sarracenia purpurea), cotton-grasses (Eriophorum spissum and E. virginicum), sedges (Carex spp.), beak rush (Rhynchospora alba), and sundews (Drosera intermedia and $D$. rotundifolia).

Swamps. These treed wetlands are based on mineral soils, although some organic soils may also be present.

Coniferous-tree swamps are the most prevalent with stands of black spruce (Picea mariana) or a mixture of balsam-fir (Abies balsamea) and eastern white cedar (Thuja occidentalis). The ground is quite uneven because of mounds surrounding the tree bases and because of hummocks produced by various moss species and liverworts, between which lie shallow pools of standing or slowly moving water.

Deciduous-tree swamps are less frequent and are dominated by black ash (Fraxinus nigra) and red maple (Acer rubrum). Their floors are covered with a sodden layer of fallen leaves.

Coniferous-tree swamps are highly productive sites for herbaceous plants such as naked mitrewort (Mitella nuda), bitter cress (Cardamine 
pensylvanica), smaller enchanter's nightshade (Circaea alpina), dwarf raspberry (Rubus pubescens), jewelweed (Impatiens capensis), early coral-root (Corallorhiza trifida), tall northern green orchid (Platanthera hyperborea), blunt-leaf orchid ( $P$. obtusata), kidney-leaved violet (Viola renifolia), shinleaf (Pyrola elliptica), and many species of mosses.

The most frequent ferns of swamplands are sensitive fern (Onoclea sensibilis), cinnamon fern (Osmunda cinnamomea), and royal fern (Osmunda regalis); these species are often associated with swampy mixed forest. Along the edges of some swamps, wood nettle (Laportea canadensis) and swamp red currant (Ribes triste) are found.

Dead-tree swamps. These wooded areas have been flooded by beaver dams or other obstructions to flowing water. If the water is deep $(40 \mathrm{~cm}$ or more), floating and other aquatic plants may be present. Duckweed (Lemna minor) sometimes covers much of the surface. When the water is shallow, the ground is seen as a series of grassy hummocks pocked with water holes, which may support aquatic or wetland species.

Wet thickets. Numerous along the edges of bogs and swamps, these thickets may be dominated either by speckled alder (Alnus rugosa) or willows (Salix spp.) or by mountain holly (Nemopanthus mucronata), wild-raisin (Viburnum cassinoides), winterberry (Ilex verticillata), or chokeberry (Aronia melanocarpa).

Marshes. Like swamps, marshes receive nutrients from mineral soils as well as from some organic soils, but trees are absent.

Cat-tail marshes, which occur around ponds and fill in the bays of lakes, are the most extensive and fastest growing. Water seeps through their dense growth, lies in pools, or flows through in winding streams. In these marshes, cat-tails (Typha latifolia and occasionally $T$. angustifolia) dominate. Horsetail (Equisetum fluviatile), water-arum (Calla palustris), wild iris (Iris versicolor), several sedges (Carex spp.), and a spike-rush (Eleocharis palustris) frequently share the sites.

Reed marshes are less dense than cat-tail marshes and are usually dominated by species of bulrush (Scirpus), most often by soft-stem bulrush (S. validus). Sometimes the horsetail (Equisetum fluviatile) will be equally abundant.

Associated with both types of marshes are the wild iris (Iris versicolor), sedges (such as Carex oligosperma), pickerel-weed (Pontederia cordata), bur-reed (Sparganium chlorocarpum), and in open-water areas, white water-lily (Nymphaea odorata), yellow water-lily (Nuphar pumila), water-shield (Brasenia schreberi), pondweeds (Potamogeton spp.), water-milfoils (Myriophyllum spp.), grasses (Glyceria borealis and G. canadensis), and rushes (Juncus articulatus and J. canadensis). 


\section{OPEN-WATER HABITATS}

With its more than 600 lakes and numerous streams, Haliburton has a bountiful supply of habitats for plants of open water. In larger lakes they are usually found along shores or in quiet bays, but in small lakes and ponds they may extend to much of the open-water area. In rivers plants are numerous in the backwaters, while some species such as tapegrass (Vallisneria americana) may inhabit swift currents. Starwort (Callitriche verna) and waterweed (Elodea canadensis) occur in slower streams. The pondweeds (Potamogeton spp.), of which 21 species appear on the list, are represented in all these habitats. In quiet waters, 8 species of bladderworts (Utricularia spp.) and 6 species of water-milfoils (Myriophyllum spp.) occur. A few bladderworts and coontails (Ceratophyllum spp.) can be seen floating freely in masses unattached to the bottom during part of their life cycles.

\section{OTHER HABITATS}

In addition to wetland and open-water areas, other damp to wet habitats harbour moisture-loving plants: shores, ditches, depressions, and wet meadows also contribute to the list of flora of the county of Haliburton.

As Haliburton land is largely wooded with many outcroppings of exposed rock, the amount of open ground with soil cover is limited. Old fields, clearings, and other untreed locations where soil is adequate for plant growth, are not common. Disturbed sites along roadsides, however, provide opportunities for a number of species that prefer open habitats. Viper's bugloss (Echium vulgare), chicory (Cichorium intybus), fireweed (Epilobium angustifolium), goldenrods (Solidago spp.), hawkweeds (Hieracium spp.), plantains (Plantago spp.), and thistles (Cirsium arvense and C.vulgare) are among the plants of open habitats often found on the roadsides of Haliburton. Many primary and secondary roads run through wetlands where typical swamp, bog, or marsh species often grow along their edges. Bush roads provide partial shade and moist soil for a further group of plants. 


\section{METHODS}

\section{COLLECTING AND COLLECTIONS}

The annotated list that follows is based primarily on the $2100+$ specimens that were collected and identified by the authors during the period 1976-1984. We have also examined specimens collected by others and deposited in the herbaria cited in the list. While most collections were made since 1970, a few are of earlier date, notably those of Hubert $\mathrm{H}$. Brown in the 1930's and of William Scott in 1891.

The habitats described for each taxon on the list refer specifically to the locations in which they were found in the county of Haliburton and do not include habitat data for the same taxa from other sources.

No plants under cultivation are recorded. Many species, however, are of European or Asiatic origin. These are now naturalized in North America and are indicated by a (+) at the end of the habitat description. A few plants considered to be escapes from cultivation are noted as such.

\section{TERRITORY COVERED}

Of the 23 townships of the county, 22 are represented on the list, since the remaining township (Eyre) was inaccessible to us for collecting purposes. A new township, Bicraft, within the boundaries of Cardiff Township was in the process of being established, but for our purposes the area has been included in Cardiff.

While field trips were planned to provide as wide a geographical coverage as possible, emphasis was placed on seeking out the greatest variety of habitats. Consequently, some townships received more attention than others, and are represented by more taxa, while some common species were undercollected.

\section{IDENTIFICATION AND NOMENCLATURE}

For identification, The New Britton and Brown Illustrated Flora (Gleason, 1952), Gray's Manual of Botany, 8th edition (Fernald, 1950), and Manual of Vascular Plants (Gleason and Cronquist, 1963) were used. Where alternative family names suggested by Article 18 of the International Code of Botanical Nomenclature (Stafleu et al., 1972) are used, those they replace follow in parentheses. Two families of recent authors, Potamogetonaceae and Pyrolaceae, are included. Family names appear in the Britton and Brown sequence, but for ease of reference, genera are listed in alphabetical order within each 
family. In addition to these basic texts, some more recent authors were also studied: Britton $(1984,1985)$ for ferns and fern allies, Voss (1972) for monocots, Voss (1985) for some dicots, Luer (1975) and Whiting and Catling (1986) for orchids, Dore and McNeill (1980) for grasses, Soper and Heimburger (1982) for shrubs, and Sell and West (1976) for Hieracium. Additional publications that were consulted are listed at the end of this volume. In some taxa, the nomenclature has been updated based on these references. Common names were selected from texts and field guides, with an attempt to use those that are best known and yet suitable. Authorities for many taxa in the list were checked in Morton and Venn (1989), and amended to accord with their usage.

\section{VOUCHER SPECIMENS}

The authors' voucher specimens have been deposited in the University of Toronto Vascular Plant Herbarium (TRT), now located in the Botany

Department of the Royal Ontario Museum, Toronto. In the annotated list, the location of a voucher specimen for each township is given to assist those wishing to examine specimens or produce distribution maps. With rare species, however, a second specimen is also listed when obtained from an additional location in the same township.

On the voucher specimens collected by the authors, the geographic coordinates represent the locations of collecting stations; specimens were collected up to approximately one kilometre from these stations.

\section{ABUNDANCE RATINGS}

Abundance ratings are based on voucher specimens, sight records, and fieldtrip notes. The following is a list of the terms used:

Common: Numerous and widely distributed in the county.

Fairly common: Found in seven or more locations.

Uncommon: Found in three to six locations.

Rare: Found in one or two locations.

Plants that are listed as rare in Ontario are the native species recorded by Argus et al. (1982-1987). 


\title{
ACRONYMS, ABBREVIATIONS, AND SYMBOLS
}

\section{HERBARIUM ACRONYMS \\ (HOLMGREN AND KEUKEN, 1974)}

\begin{abstract}
APM Algonquin Park Museum, Algonquin Park.
CAN Vascular Plant Section, National Herbarium of Canada, National Museum of Canada, Ottawa.

DAO Vascular Plant Herbarium, Department of Agriculture, Ottawa.

DFB Private herbarium of D. F. Brunton, Ottawa.

MICH Herbarium of the University of Michigan, Ann Arbor.

OAC Department of Botany, University of Guelph.

PENN University of Pennsylvania, Philadelphia.

REW Private herbarium of R. Emerson Whiting, Toronto.

TRT Vascular Plant Herbarium, Department of Botany, Royal Ontario Museum, Toronto.

TRTE Erindale College, University of Toronto.

UWO Herbarium, Department of Plant Sciences, University of Western Ontario, London.

WAT Herbarium, University of Waterloo.
\end{abstract}

\section{TOWNSHIP ABBREVIATIONS}

$\begin{array}{llllll}\text { Ans } & \text { Anson } & \text { Hav } & \text { Havelock } & \text { Mnd } & \text { Minden } \\ \text { Brt } & \text { Bruton } & \text { Hbn } & \text { Harburn } & \text { Mon } & \text { Monmouth } \\ \text { Cly } & \text { Clyde } & \text { Hct } & \text { Harcourt } & \text { Ngt } & \text { Nightingale } \\ \text { Crd } & \text { Cardiff } & \text { Hin } & \text { Hindon } & \text { Shb } & \text { Sherborne } \\ \text { Dud } & \text { Dudley } & \text { Liv } & \text { Livingston } & \text { Sno } & \text { Snowdon } \\ \text { Dys } & \text { Dysart } & \text { Lut } & \text { Lutterworth } & \text { Stn } & \text { Stanhope } \\ \text { Gfd } & \text { Guilford } & \text { Lwr } & \text { Lawrence } & & \\ \text { Glm } & \text { Glamorgan } & \text { McC } & \text { McClintock } & & \end{array}$

\section{OTHER ABBREVIATIONS AND SYMBOLS}

auct. non [auctorum non] (preceding name of author): Not described by.

f. (preceding a scientific name): Forma.

f. (following a name of author): Son.

S.R.: Sight record.

subsp.: Subspecies.

var. (preceding a scientific name): Variety.

$(+)$ : Introduced to this continent from Europe or Asia. 


\section{MAPS USED IN ESTABLISHING LOCATIONS}

1. The Provisional County of Haliburton and the District Municipality of Muskoka. Ontario Ministry of Transportation and Communications, Map MTC 30201, revised 1979.

2. Maps of the National Topographic System of Canada, 1:50 000 scale.
Map 31E/1 1975 Wilberforce
Map 31E/2 1974 Haliburton
Map 31E/7 1974 Kawagama
Map 31E/8 1975 Whitney
Map 31D/15 1972 Minden
Map 31D/16 1971, 1980 Gooderham

Canada Map Office, Surveys and Mapping Branch, Department of Energy, Mines, and Resources, Ottawa, Canada K1A 0E9.

3. Physiography of the Georgian Bay-Ottawa Valley Area. Ontario Ministry of Natural Resources and Ontario Research Foundation, Map 2228, Physiography, 1975.

4. Haliburton-Bancroft Area. Province of Ontario, Department of Mines, Map 1957b, 1957. 



\section{ANNOTATED LIST OF THE VASCULAR PLANTS OF THE COUNTY OF HALIBURTON}

\section{FERN ALLIES}

LYCOPODIACEAE

Lycopodium annotinum $\mathrm{L}$.

CLUBMOSS FAMILY

Bristly clubmoss

Common. Dry coniferous and damp mixed woods.

TRT-Crd, Hct, Liv, Mnd, Shb; S.R.-Dys.

Lycopodium clavatum $\mathbf{L}$.

Staghorn clubmoss

Common. Wet to dry woods, grassy banks, and moss-covered fields.

DFB-Lwr; TRT-Crd, Cly, Dys, Hct, Hin, McC, Mnd, Mon; S.R.-Glm.

Lycopodium dendroideum Michaux

L. obscurum L. var. dendroideum (Michaux) D. C. Eaton

Tree clubmoss

Common. Coniferous, deciduous, or mixed woods; on damp humus.

TRT-Brt, Crd, Dud, Hbn, Hct, Mnd, Shb, Stn.

Lycopodium digitatum A. Braun Ground-cedar, Running pine clubmoss L. complanatum L. var. flabelliforme Fern.

Common. Damp mixed or deciduous woods, and occasionally open mossy turf.

TRT-Crd, Hct, Mnd, Mon, Shb, Stn.

Lycopodium inundatum $\mathrm{L}$.

Bog clubmoss

Uncommon. Damp to wet sand-and-gravel flats.

APM-Lwr; TRT-Hbn; S.R.-Dys, Shb.

Lycopodium lucidulum Michaux

Common. Mixed or deciduous woods, on damp leafy humus.

APM-Ngt; TRT-Crd, Gfd, Hbn, Hct, McC, Mnd, Shb, Stn.

Lycopodium obscurum $\mathbf{L}$.

Ground-pine var. isophyllum Hickey

Common. Mixed woods, on humus over sandy soil.

TRT - Brt, Crd, Gfd, Hbn, Mnd, Shb, Stn. 
Lycopodium sabinifolium Willd.

Savin-leaved clubmoss

Rare. One location. Old field edged with conifers.

TRT-Hin.

Lycopodium tristachyum Pursh

Ground-cedar

Uncommon. Open sandy areas and in low vegetation.

APM-Ngt; TRT-Crd, Gfd, Hct, Shb.

SELAGINELLACEAE

Selaginella rupestris (L.) Spring

Rare. One location. Rocky hillside.

TRT-Mnd.

ISOETACEAE

Isoetes echinospora Durieu

Common. Quiet rivers and lakes at 5 to $100 \mathrm{~cm}$ depth on sandy, gravelly, or occasionally mucky bottoms.

TRT-Gfd, Hbn, Shb, Stn.

Isoetes macrospora Durieu

Quillwort

Uncommon. Lake bottoms at 1 to $6.5 \mathrm{~m}$ depth.

OAC-Gfd, Hin, McC, Shb.

f. hieroglyphica (A. A. Eaton) N. E. Pfeiff

Rare. One location. Sandy-silty shallow bay.

CAN-Shb.

EQUISETACEAE

Equisetum arvense $\mathrm{L}$.

HORSETAIL FAMILY

Common horsetail

Common. Open sandy sites, roadsides, and damp ditches.

TRT-Hct, Mnd, Stn; S.R.-Lut.

Equisetum fluviatile $\mathbf{L}$.

Water horsetail

Common. Shallow water to $1 \mathrm{~m}$ depth on edges of streams, rivers, and bays; often in large dense colonies.

TRT-Crd, Gfd, Hct, Lut, Sno, Stn.

Equisetum hyemale $\mathbf{L}$.

Rough scouring-rush

var. affine (Engelm.) A. A. Eaton

Fairly common. Dry banks and roadsides.

TRT-Crd, Dys, Hct; S.R.-Mnd.

Equisetum scirpoides Michaux

Dwarf scouring-rush

Rare. Two locations. Damp woods, in small colonies.

TRT-Crd, Sno. 
Equisetum sylvaticum $\mathbf{L}$.

Woodland horsetail

Common. Low, damp or wet areas at edges of woods, on sandy or gravelly soil.

TRT-Crd, Dys, Hbn, Hct, Stn; S.R.-Mnd.

Equisetum variegatum Schleicher

Variegated horsetail Uncommon. Open, wet areas and damp ditches, on sandy or silty soils; colonies often dense.

TRT-Crd, Shb; S.R.-Lut.

\section{FERNS}

OPHIOGLOSSACEAE

Botrychium dissectum Sprengel

var. obliquum (Muhlenb.) Clute

Rare. Two locations. Open, dry grassy sites.

TRT-Dys, Shb.

Botrychium matricariifolium A. Braun ex Koch

Common. Deciduous or mixed woods and treed fields.

TRT-Dys, Mnd, Mon, Shb.

Botrychium multifidum (S. Gmelin) Rupr.

Common. Woodlands and edges of woods, on sandy soils.

TRT - Dys, Crd, Glm, Hbn, Hct, Mnd, Mon, Shb.

var. intermedium (D. C. Eaton) Farw.

Common. Woodlands and meadows, on sandy or gravelly soils.

TRT-Crd, Glm, Hct, Mnd, Shb.

Botrychium simplex E. Hitchc.

var. tenebrosum (A. A. Eaton) Clausen

Rare. Two locations. Damp edges of streams.

CAN-Crd; TRT-Shb.

Botrychium virginianum (L.) Sw.

Rattlesnake fern

Common. Deciduous woods, on humus.

TRT-Crd, Hbn, Hct, Lut, Mon, Stn; S.R.-Dys.

OSMUNDACEAE

ROYAL FERN FAMILY

Osmunda cinnamomea $\mathrm{L}$.

Common. Wet woods, shores of ponds and streams, and damp ditches.

TRT-Dud, Gfd, Hct, Liv, Mnd, Shb; S.R.-Crd. 
Common. Damp or wet woodland sites, and edges of lakes, streams, and swamps.

TRT-Crd, Gfd, Hbn, Hct, Hin, Mnd, Shb; S.R.-Cly.

Osmunda regalis $\mathrm{L}$.

Royal fern

Common. Margins of lakes and streams, just above or in the water; often in large dense colonies.

TRT -Crd, Dud, Gfd, Hct, Liv, Stn.

POLYPODIACEAE

POLYPODY FAMILY

This large family has been divided into several smaller ones by recent authors, but since changes may still be underway, Polypodiaceae is presented intact here. For information on new names the reader is referred to Britton (1985).

Adiantum pedatum $\mathrm{L}$.

Maidenhair fern

Common. Deciduous or mixed woods, in leaf-covered humus.

TRT-Crd, Gfd, Hct, Hin, Lut, McC, Mnd, Mon.

Asplenium trichomanes $\mathrm{L}$.

Rare. One location. Base of calcareous rock cliff.

Maidenhair spleenwort

TRT-Crd.

Athyrium filix-femina (L.) Roth

var. michauxii Mett.

Upland lady fern

Common. Damp ditches and moist open sites; in large or small

dense colonies.

TRT-Cly, Crd, Dud, Gfd, Hct, Mnd, Shb, Stn.

Athyrium pycnocarpon (Sprengel) Tidestrom

Rare. One location. Sugar maple forest.

TRT-Crd.

Athyrium thelypterioides (Michaux) Desv.

Silvery spleenwort

Fairly common. Damp or wet woods, in humus.

APM-Brt; TRT-Crd, Dud, Gfd, Hbn, Mon.

Cystopteris bulbifera (L.) Bernh.

Narrow-leaved spleenwort

Common. Damp areas, usually in woods; in clay or humus.

TRT-Crd, Dud, Hct, Mnd, Stn. 
Cystopteris tenuis (Michaux) Desv.

Northern fragile fern

Common. Open rocky hillsides, rock crevices, and occasionally in dense woods.

TRT-Crd, Dys, Hav or Shb, Hbn, Hct, Mnd, Stn.

Dennstaedtia punctilobula (Michaux) Moore

Hay-scented fern

Common. Dry banks, roadsides, and damp shores; often in large colonies.

CAN-Shb; DAO-Hct, Shb; TRT-Cly, Crd, Hbn, Hct, Hin, McC,

Shb, Sno.

Dryopteris carthusiana (Villars) H. P. Fuchs

Spinulose wood fern

D. spinulosa (O. F. Muell.) Watt

D. austriaca (Jacq.) Woynar var. spinulosa (O. F. Muell.) Fiori

Common. Damp mixed or deciduous woods and swamps.

APM-Ngt; TRT-Dud, Gfd, Hbn, Hct, Mnd; S.R.-Crd, Lut.

Dryopteris cristata (L.) A. Gray

Crested shield fern

Common. Damp ditches and meadows.

TRT_Crd, Dys, Hct, Shb, Stn; S.R.-Cly, Mon.

Dryopteris fragrans $(\mathrm{L}$.$) Schott$

Fragrant wood fern

Rare. Two locations. Rocky cliffs.

APM-Cly, Ngt.

Dryopteris goldiana (Hook.) A. Gray

Goldie's fern

Rare. One location. Moist woods.

TRT-Mnd.

Dryopteris intermedia (Muhlenb.) A. Gray

Evergreen wood fern

D. spinulosa (O. F. Muell.) Watt var. intermedia (Muhlenb.) Underw.

D. austriaca (Jacq.) Woynar var. intermedia (Muhlenb.) Morton

Common. Moist areas of coniferous and dense deciduous woods.

DAO-Hct, Shb, Stn; DFB-Lut; TRT-Crd, Dys, Hct, Shb, Stn.

Dryopteris marginalis (L.) A. Gray

Marginal shield fern

Common. Mixed or deciduous woods and wooded banks.

TRT-Crd, Gfd, Hbn, Hct, Shb, Stn.

f. elegans (J. Robins.) F. W. Gray

Rare. One location. Damp valley in sugar maple forest.

TRT-Crd.

Dryopteris $\times$ boottii (Tuckerm.) Underw.

Hybrid fern

$=D$. cristata $\times D$. intermedia

Rare. One location.

TRT-Stn. 
$=D$. carthusiana $\times$ D. intermedia

D. spinulosa (O. F. Muell.) Watt var. fructuosa (Gilbert) Trudell

D. austriaca (Jacq.) Woynar var. fructuosa (Gilbert) Morton

Uncommon. Moist or wet, mixed or deciduous woods.

TRT - Hbn, Hct, Shb.

Gymnocarpium dryopteris (L.) Newm.

Oak fern

Dryopteris disjuncta (Ledeb.) Morton

Common. Deciduous or mixed woods and soil pockets on rocks.

APM-Ngt; TRT-Crd, Gfd, Hct, Mnd, Shb; S.R.-Crd.

Matteuccia struthiopteris (L.) Todaro

Ostrich fern

Common. Damp roadsides, swampy sites, and wet ditches.

TRT-Hct, Mnd, Stn; S.R.-Brt, Crd, Lut.

Onoclea sensibilis $\mathbf{L}$.

Sensitive fern

Common. Damp to wet areas: ditches, swamps, and shores.

APM-Ngt; TRT-Crd, Gfd, Hct, Hin, Mnd, Shb, Stn; S.R.-Cly, Glm, Lut, Sno.

Phegopteris connectilis (Michaux) Watt

Northern beech fern

Thelypteris phegopteris (L.) Slosson

Dryopteris phegopteris (L.) Christens.

Common. Damp woods and edges of lakes and streams; often in dense colonies on banks.

APM-Ngt; CAN-Hct, Shb; TRT-Crd, Dud, Gfd, Mnd, Shb.

\section{Polypodium virginianum $\mathbf{L}$.}

P. vulgare L. var. virginianum (L.) A. A. Eaton

Common polypody

Common. Rocky banks, and rocky outcrops in woods.

DFB_Lwr; TRT_Crd, Dys, Gfd, Hbn, Hct, Mnd, Ngt, Shb.

Polystichum acrostichoides (Michaux) Schott

Christmas fern

Common. Deciduous or mixed woods, usually in damp humus.

APM-Brt, Lwr, Ngt; TRT-Crd, Hct, Hin, Mnd, Mon, Stn.

Pteridium aquilinum (L.) Kuhn

Bracken fern

Common. Open barren sites, dry ditches, and partly treed fields;

frequently in large dense colonies.

TRT-Crd, Hct, Mnd; S.R.-Ans, Dys, Glm, Hin, Shb.

Thelypteris noveboracensis (L.) Nieuwl.

New York fern

Common. Low, damp woods and clearings, on rich humus soil.

TRT-Cly, Crd, Gfd, Hct, Hin, McC, Mnd, Shb, Stn. 
Uncommon. Edges of creeks and streams among grasses and sedges.

TRT -Crd, Hin, Mnd; S.R.-Lut.

Woodsia ilvensis (L.) R. Br.

Fairly common. Rock ledges of cliffs and shores.

DAO-Crd, McC; TRT-Crd, Hin, Mnd, Stn.

Woodwardia virginica (L.) Smith

Virginia chain fern

Rare. Two locations. One very large colony bordering a small lake, and a smaller one bordering a sphagnum mat island; both growing densely with lower stems in water.

TRT-Crd, Stn.

\section{CONIFERS}

TAXACEAE

YEW FAMILY

Taxus canadensis Marshall

American yew

Fairly common. Low clearings in mixed woods, and wooded slopes.

APM-Brt, Lwr; TRT-Mnd, Stn.

PINACEAE

PINE FAMILY

Abies balsamea (L.) Miller

Balsam-fir

Common. Mixed woods and lakeshores; scattered or in pure stands.

TRT-Mnd, Shb; S.R.-Crd, Hct, Sno.

Larix laricina (Du Roi) K. Koch

Tamarack

Common. Low, damp areas: open or treed bogs and damp shore banks.

TRT_Dud, Hct, Mnd, Stn; S.R.-Ans, Glm, Sno.

Picea glauca (Moench) Voss

White spruce

Common. Mixed woods and pure stands; in high, dry sandy areas and low, damp areas.

TRT_Dud, Mnd, Shb; S.R.-Crd, Dys, Gfd, Glm, Hin, Mon, Sno, Stn.

Picea mariana (Miller) Britton, Sterns, and Pogg.

Black spruce

Common. Low, damp to wet areas; scattered through coniferous woods or in large stands.

TRT-Dud, Hct, Mnd; S.R.-Dys.

Picea rubens Sarg.

Red spruce

Uncommon. Dry rocky soil and low, damp areas.

APM-Brt, Cly; DAO-Cly; DFB-Lwr. 
Common. Dry woods, and rocky ridges and shores.

TRT-Mnd, Shb.

Pinus strobus L.

White pine

Common. Rocky shores and slopes, sand flats, and moist mixed woods.

TRT-Crd, Hct, Mnd, Shb, Stn; S.R.-Ans, Dys, Glm, Hin, Mnd,

Mon, Sno.

Pinus sylvestris $\mathbf{L}$.

Uncommon. Reforested sites and adjacent areas; introduced and

Scotch pine spreading. (+)

TRT-Mnd, Stn.

Tsuga canadensis (L.) Carrière

Eastern hemlock

Common. Coniferous or mixed woods and lakeshores; often in large stands.

TRT-Hct, Shb, Sno; S.R.-Ans, Dys, Gfd, Glm, Hin, Mnd.

\section{CUPRESSACEAE}

Juniperus communis $\mathrm{L}$.

CYPRESS FAMILY var. depressa Pursh

Common juniper

Fairly common. Rocky shores and open fields.

APM-Ngt; TRT-Hin; S.R.-Mnd.

Thuja occidentalis L.

Eastern white cedar

Common. Shores of lakes, rocky outcrops, and damp woods.

TRT-Hct, Mnd, Shb; S.R.-Ans, Crd, Gfd.

\section{FLOWERING PLANTS MONOCOTYLEDONS}

TYPHACEAE

Typha angustifolia $\mathrm{L}$.

CAT-TAIL FAMILY

Fairly common. Wet ditches and marshes; often growing with Typha

latifolia.

TRT -Crd, Dys, Hct, Lut, Mnd.

Typha latifolia L.

Common cat-tail

Common. Marshes, wet ditches, and edges of ponds and bays; often in very large colonies.

TRT-Crd, Dys, Hct, Mnd, Stn; S.R.-Ans, Cly, Lut. 
Common. Near shores in water 10 to $30 \mathrm{~cm}$ deep, and in wet ditches and beaver meadows; in dense colonies.

TRT-Dys, Hct, Lut, Mnd, Shb, Stn; S.R._Mon.

Sparganium angustifolium Michaux

Bur-reed

Uncommon. Edges of lakes and streams; shallow water on sandy bottoms. TRT-Shb, Sno; S.R._Lut.

Sparganium chlorocarpum Rydb.

Bur-reed

Common. Wet flats and meadows, and shallow water on edges of streams and marshes.

APM-Ngt; TRT-Crd, Dud, Gfd, Hct, Mnd; S.R. - Hin, Shb.

\section{f. acaule (Beeby) E. Voss}

Fairly common. Found among Sparganium chlorocarpum or in separate colonies.

TRT-Dud, Shb; S.R.-Mnd.

Sparganium fluctuans (Morong) Robinson

Floating bur-reed

Common. Offshore in lakes and streams in water up to $1 \mathrm{~m}$ deep.

TRT_Crd, Dys, Gfd, Hct, Hin, Liv, McC.

Sparganium minimum (Hartman) Fries

Bur-reed

Rare. One location. Edge of bay adjoining marsh, in quiet shallow water.

TRT-Mnd.

POTAMOGETONACEAE

(NAJADACEAE in part; ZOSTERACEAE)

Potamogeton alpinus Balbis

PONDWEED FAMILY

Uncommon. Shallow bays in water 30 to $90 \mathrm{~cm}$ deep; locally numerous.

TRT-Crd, Dys, Mnd.

Potamogeton amplifolius Tuckerman

Large-leaf pondweed

Common. Open water 60 to $90 \mathrm{~cm}$ deep, and shores of lakes, ponds, and rivers.

TRT-Crd, Dys, Hct, Mnd, Stn.

Potamogeton bicupulatus Fern.

Narrow-leaved pondweed

Rare. Two locations. Single specimens floating alone or with Potamogeton spirillus near shores of small lakes. Rare in Ontario (Argus et al., 19821987).

TRT-Hin, Shb. 
Rare. One location. Near shore of lake in water $4 \mathrm{~cm}$ deep; in small colony. Rare in Ontario (Argus et al., 1982-1987).

TRT-Hin.

Potamogeton epihydrus Raf.

Common. Lakes, ponds, and streams; in water up to $1 \mathrm{~m}$ deep.

TRT_Crd, Dys, Glm, Hct, Hin, McC, Shb; S.R.-Mon.

Potamogeton foliosus Raf.

Narrow-leaved pondweed

Rare. Two locations. On soft bottom of shallow lake at $0.5 \mathrm{~m}$ depth, and in shallow creek.

TRT -Crd, Dys.

Potamogeton friesii Rupr.

Narrow-leaved pondweed

Rare. One location. Quiet shallow water 60 to $80 \mathrm{~cm}$ deep; in small dense colony.

TRT-Crd.

Potamogeton gramineus $\mathrm{L}$.

Variable pondweed

Common. Quiet or flowing water up to $60 \mathrm{~cm}$ deep, on sand or soft muck bottoms.

TRT-Crd, Dys, Gfd, Hct, Mnd, Sno.

\section{Potamogeton illinoensis Morong}

Pondweed

Rare. Two locations. Swift-flowing rivers.

TRT-Gfd, Sno.

Potamogeton natans $\mathrm{L}$.

Floating pondweed

Common. Small lakes, ponds, and open water in marshes.

TRT-Dys, McC, Mnd; S.R.-Crd, Hin, Shb.

Potamogeton oakesianus Robb.

Floating pondweed

Uncommon. Quiet waters near shores of ponds and shallow bays.

DFB-McC; TRT-Lut, Shb.

Potamogeton obtusifolius Mert. and Koch

Narrow-leaved pondweed

Rare. One location. Small lake with silty sand bottom.

TRT-Dud.

Potamogeton pectinatus $\mathrm{L}$.

Sago pondweed

Uncommon. Shallow water near shores in small lakes.

TRT-Lut, 2 Mnd. 
Potamogeton perfoliatus $\mathrm{L}$.

Pondweed

Rare. Two locations. Slow-moving stream, and in lake in water $1 \mathrm{~m}$ deep.

TRT-Mnd, Stn.

Potamogeton praelongus Wulfen

Pondweed

Rare. One location. Deep water of river.

UWO-Lut.

Potamogeton pusillus $\mathbf{L}$.

Narrow-leaved pondweed

Common. Shallow waters of small lakes and bays, and quiet backwaters of streams; floating in dense masses.

TRT_Crd, Dys, Mnd; S.R.-Hin, Lut, Mon.

\section{Potamogeton pusillus $\mathbf{L}$.}

var. tenuissimus (Mert. and Koch) Fern.

$P$. berchtoldii Fieber

Uncommon. In small slow stream, and offshore in small lakes.

TRT -2 Dys, McC.

Potamogeton richardsonii (A. Bennett) Rydb.

Pondweed

Fairly common. Shallow, quiet waters and edges of flowing streams; in small to large colonies.

TRT-Gfd, Mnd, Sno.

Potamogeton robbinsii Oakes

Pondweed

Common. Streams through marshes, and offshore in lakes in water up to $1 \mathrm{~m}$ deep; colonies usually dense.

TRT -Crd, Dys, Lut, Mnd.

Potamogeton spirillus Tuckerman

Snailseed pondweed

Fairly common. Quiet water at edges of streams and bays.

TRT-Crd, Dys, Hin, Mnd.

Potamogeton zosteriformis Fern.

Flat-stemmed pondweed

Common. Quiet waters up to $1.5 \mathrm{~m}$ deep: in lakes, slow streams, and open water of swamps.

TRT-Crd, Dud, Dys, Lut, Mnd.

NAJADACEAE

PONDWEED FAMILY

Najas flexilis (Willd.) Rostkov and W. Schmidt

Naiad

Common. Shallow water of small lakes and creeks; usually in large dense colonies.

TRT-Crd, Mnd, Shb; S.R.-Lut. 
Rare. One location. Off boggy shore in small lake.

TRT-McC.

\section{ALISMATACEAE}

Alisma plantago-aquatica $\mathrm{L}$.

WATER-PLANTAIN FAMILY

Water-plaintain

Fairly common. Edges of streams and ponds, and wet ditches.

TRT-Crd, Hbn, Sno, Stn.

Sagittaria cuneata E. Sheldon

Northern arrowhead

Fairly common. Quiet shallow or flowing water; leaves emersed or floating. TRT-Crd, Dud, Hbn, Hct.

\section{Sagittaria graminea Michaux}

Arrowhead

Rare in flower or fruit. Rosettes of leaves, probably of this species, seen frequently in shallow water on sandy bottoms.

TRT-Stn.

Sagittaria latifolia Willd.

Arrowhead

Common. Offshore in, or on edges of, lakes, ponds, and streams; usually in colonies of either broad-leaved or narrow-leaved plants, though both leaf widths can occur on the same plant.

TRT-Crd, Dys, Hav, Hct, Mnd, Shb, Stn; S.R.-Hin, Lut, Mon.

\section{HYDROCHARITACEAE}

Elodea canadensis Rich. ex Michaux

FROG'S-BIT FAMILY

Waterweed

Anacharis canadensis (Rich.) Planchon

Common. Flowing streams and quiet water up to $1 \mathrm{~m}$ deep; in large dense colonies.

TRT-Crd, Mnd; S.R.-Lut.

Vallisneria americana Michaux

Tapegrass

Common. Quiet or flowing water of lakes and rivers.

TRT-Dys, Mnd, Stn.

POACEAE

GRASS FAMILY

(GRAMINEAE)

Agropyron repens (L.) P. Beauv.

Quack grass

Common. Sandy roadsides and waste places. $(+)$

TRT-Hbn, Mnd, Stn. 
Agropyron trachycaulum (Link) Malte

var. glaucum (Pease and Moore) Malte

Rare. One location. Soil pockets on rocky cliff.

TRT - Stn.

var. novae-angliae (Scribner) Fern.

Rare. Two locations. Sand-and-gravel roadsides.

TRT - Hct, Stn.

Agrostis gigantea Roth

Common. Sand flats, waste ground, and roadsides. (+)

Redtop grass

TRT-Hct, Mnd, Stn; S.R.-Sno.

Agrostis perennans (Walter) Tuckerman

Autumn bent grass

Uncommon. Dry rocky, to wet sandy sites.

TRT-Hct, Mnd; S.R.-Shb.

Agrostis scabra Willd.

A. hyemalis (Walter) Britton, Sterns, and Pogg.

Tickle grass

Fairly common. Moss-covered rocks, wet logs in shallow water, and roadsides.

TRT-Cly, Dys, Stn; S.R.-Lut.

Agrostis stolonifera L.

Creeping bent grass

Uncommon. Dry sandy, or wet grassy edges of roads.

TRT-Dys, Sno.

Alopecurus aequalis Sobol.

Short awn foxtail

Uncommon. A variety of open, damp or wet locations.

DAO-2 Crd; TRT-Mnd.

Anthoxanthum odoratum $\mathrm{L}$.

Sweet vernal grass

Uncommon. Open, flat roadsides; on moist or dry sand and gravel. (+)

TRT-Hin, Lut, Sno.

Brachyelytrum erectum (Schreber) P. Beauv.

Leafy wood grass

Common. Woods, damp roadsides, and wet sand flats.

APM-Cly; TRT-Dud, Hct, Mnd; S.R.-Lut, Sno.

Bromus ciliatus $\mathrm{L}$.

Fringed brome grass

Common. Sandy roadsides and grassy ditches.

TRT-Cly, Dys, Sno.

Bromus inermis Leysser

Smooth brome grass

Common. Gravel roadsides and open rocky areas. (+)

APM-Ngt; TRT-Mnd, Sno, Stn; S.R.-Dys, Lut. 
Rare. Two locations. Dry roadside and grassy hillside. (+)

TRT-Hct, Mnd.

Calamagrostis canadensis (Michaux) P. Beauv.

Common. Swampy areas, damp ditches, and hillsidu.

TRT-Cly, Crd, Hct, Mnd.

Calamagrostis inexpansa A. Gray

Northern reed grass

Rare. One location. Rocky shoreline.

TRT-Mnd.

Cinna latifolia (Trevin) Griseb.

Common. Thin woods and grassy roadsides.

Blue joint reed grass

TRT - Brt, Crd, Het, Hin, Mnd.

Dactylis glomerata L.

Orchard grass

Common. Open sandy areas; frequently on disturbed land. (+)

TRT -Dud, Dys, Gfd, Hct.

Danthonia spicata (L.) P. Beauv. ex Roemer and Schultes

Poverty grass Common. Sandy, dry open sites.

TRT-Hbn, Hct, Mnd; S.R.-Dys.

Deschampsia flexuosa (L.) Trin.

Hair grass

Uncommon. Woods, rocky sites, and mossy banks.

TRT-Hin, Lut, Shb.

Digitaria ischaemum (Schreber) Muhlenb.

Smooth crab grass

Uncommon. Sandy and gravelly roadsides.

TRT-Crd, 2 Mnd.

Digitaria sanguinalis (L.) Scop.

Rare. One location. Open disturbed site. (+)

Nodding wood grass

TRT-Mnd.

Echinochloa crusgalli (L.) P. Beauv.

Barnyard grass

Uncommon. Dry waste areas. (+)

APM-Ngt; TRT -2 Dys.

Echinochloa wiegandii (Fassett) McNeill

Large crab grass and Dore

Fairly common. Dry roadsides.

TRT-Crd, Dys, Hin; S.R.-Shb. 
Elymus hystrix L.

Hystrix patula Moench

f. hystrix

Uncommon. Grassy slopes and gravel flats.

TRT-Dys, 2 Stn.

\section{f. bigelovianus (Fern.) Dore}

Uncommon. Sandy and grassy sites, occasionally in woods.

TRT - 2 Dys; S.R.-Lut.

Elymus virginicus $\mathrm{L}$.

Virginia wild rye-grass

Uncommon. Damp habitats: woods, flats, and stream borders.

TRT-Lut, Mnd, Sno.

Festuca arundinacea Schreber

Tall fescue

Uncommon. Roadsides: in dense grass on sand and gravel. (+)

TRT-Crd, Stn; S.R.-Sno.

Festuca longifolia Thuill.

Hard fescue

Rare. Two locations. Coniferous woods and dry parking lot. (+)

TRT-Crd, Hin.

\section{Festuca pratensis Hudson}

Rare. One location. Damp abandoned road. (+)

TRT-Sno.

\section{Festuca rubra L.}

Red fescue

Uncommon. Gravel roadsides and mossy rock ridges. (+)

TRT-Crd, Dys, Stn.

Festuca saximontana Rydb.

Rocky Mountain fescue

Rare. Two locations. Edge of hayfield and rocky shoreline.

TRT-Crd, Stn.

Glyceria borealis (Nash) Batch.

Floating manna grass

Common. Shallow water, with leaves often floating on the surface.

TRT - Hct, Mnd, Stn; S.R.-Crd.

Glyceria canadensis (Michaux) Trin.

Rattlesnake grass

Common. Damp shores of lakes and streams, and wet hollows.

TRT-Cly, Hbn, Hct, Hin, Mnd, Stn; S.R.-Dys.

Glyceria grandis S. Watson

Tall manna grass

Uncommon. River banks and dry or damp roadsides.

TRT-Dys, Hct, Stn. 
Uncommon. Gravelly or grassy road edges, and occasionally in damper areas among sedges.

TRT-Brt, Hct, Hin.

Glyceria striata (Lam.) A. Hitchc.

Common. Damp mixed woods and damp sandy roadsides.

TRT-Crd, Dud, Het, Mnd, Stn.

Hierochloe odorata (L.) P. Beauv.

Uncommon. Damp sandy roadsides.

Sweet grass

TRT-Dys, Hin, Lut.

Holcus lanatus L.

Rare. One location. Small colony in ditch with other grasses. $(+)$

Velvet grass

TRT-Dys.

Leersia oryzoides (L.) Sw.

Uncommon. Wet sandy edges of streams and rocky shores.

Cut grass

TRT-Shb, Stn; S.R.-Mnd.

Lolium perenne $\mathrm{L}$.

English rye grass

Rare. Two locations. Moist areas with other grasses. (+)

TRT-Mnd, Stn.

Milium effusum $\mathbf{L}$.

Wood millet

Uncommon. Mixed woods with leafy ground cover, and roadsides.

APM-Brt, Cly; TRT - Hin, Mnd.

Miscanthus sacchariflorus (Maxim.) Hackel

Plume grass

Rare. One location. In fence-line vegetation; escape from cultivation. $(+)$

TRT-Dys.

Muhlenbergia frondosa (Poiret) Fern.

Muhly grass

Rare. Two locations. Damp, low sites beside old bush roads.

TRT -2 Dys.

Muhlenbergia glomerata (Willd.) Trin.

Muhly grass

Rare. One location. In large patch of tall grasses beside road.

TRT-Hin.

Muhlenbergia mexicana (L.) Trin.

Common. Moist woods and damp sandy ditches.

Muhly grass

TRT-Dys, Hct, Mnd, Shb; S.R._Lut. 
Muhlenbergia uniflora (Muhlenb.) Fern.

Muhly grass

Uncommon. Moist sand flats, rocky shores, and roadsides.

TRT - Hbn, Hct, Hin, Stn; S.R.-Shb.

Nardus stricta L.

Moor mat grass

Rare. One location. Numerous clumps on dry sandy site, among low herbs and some shrubs. $(+)$

TRT-Sno.

Oryzopsis asperifolia Michaux

Fairly common. Dry, sometimes rocky woodlands.

Rice grass

TRT-Crd, Hct, Mnd, Shb, Stn.

Oryzopsis racemosa (Smith) Ricker ex A. Hitchc.

Rice grass

Uncommon. Open mixed woods, and occasionally sandy pockets on rock outcrops.

TRT-Dys, Gfd, Hct, Lut.

Panicum boreale Nash

Panic grass

Uncommon. Sandy pockets on rock point, and open grassy site on sandy soil.

TRT-2 Mnd, Sno.

Panicum capillare $\mathrm{L}$.

Witch grass

Fairly common. Swampy edges of roadsides, and waste areas.

APM-Ngt; TRT - Dys, Lut.

Panicum columbianum Scribner

Panic grass

Uncommon. Sandy sites.

APM-Ngt; TRT-Mnd, Sno; S.R.-Dys, Hin.

Panicum depauperatum Muhlenb.

Panic grass

Rare. One location. Dry, sandy open area near gravel pit.

TRT-Mnd.

Panicum implicatum Scribner

Panic grass

Common. Sand-and-gravel pits and dry disturbed land.

TRT-Gfd, Hbn, Hct, Hin, Mnd, Mon, Sno.

Panicum latifolium $\mathrm{L}$.

Broadleaf panic grass

Rare. One location. Rock ledge above lake.

TRT-Hin. 
Common. Open, dry sandy sites, wooded banks, and moist sand-andgravel ditches.

TRT - Hin, Mnd, Sno, Stn.

\section{Panicum linearifolium Scribner}

Panic grass var. werneri Scribner

Rare. One location. Old road through woods.

TRT-Lut.

Panicum miliaceum L.

Broomcorn millet

Rare. One location. Sandy and gravelly soil in thin, low patch of grasses;

escape from cultivation. (+)

TRT-Mnd.

Panicum tuckermanii Fern.

Witch grass

Rare. One location. Fencerow along road.

TRT-Sno.

Panicum xanthophysum A. Gray

Yellow panic grass

Rare. Two locations. Bank of wet ditch and damp gravel pit.

TRT -2 Shb.

Phalaris arundinacea $\mathrm{L}$.

Reed canary grass

Uncommon. Wet roadside ditches.

TRT-Dys, Stn; S.R.-Lut.

f. variegata (Par.) Druce

Ribbon grass

Uncommon. Roadside banks and ditches; escapes from cultivation. $(+)$

APM-Ngt; TRT-Hct, Stn; S.R.-Sno.

\section{Phleum pratense $\mathrm{L}$.}

Timothy

Common. Dry fields and roadsides. $(+)$

TRT-Dys, Hct, Shb, Stn.

Phragmites australis (Cav.) Trin. ex Steudel

Reed grass

Rare. Two locations. Small colony on roadside, and large dense colony at river edge.

APM-Cly; TRT -Cly, Gfd.

Poa annua $\mathrm{L}$.

Annual blue grass

Uncommon. Lawns, waste places, and road edges. (+)

APM-Brt; TRT-Mnd. 
Poa compressa L.

Canada blue grass

Common. Dry sandy, gravelly, or rocky areas. (+)

TRT-Dys, Mnd, Sno, Stn.

Poa nemoralis L.

Wood blue grass

Rare. One location. Open sandy site; one clump.

TRT-Lut.

Poa palustris L.

Fowl meadow grass

Common. Damp sandy clearings, meadows, river banks, and edges

of woods.

TRT-Mnd, Sno, Stn.

Poa pratensis $\mathrm{L}$.

Common. Sandy disturbed sites and open rocky areas. (+)

TRT -Crd, Dys, Gfd.

Poa saltuensis Fern. and Wieg.

Poa grass

Fairly common. Deciduous or mixed woods and roadside ditches.

APM-Brt; TRT-Hct, McC, Mnd, Shb.

Puccinellia fernaldii (A. Hitchc.) E. Voss

Alkali grass

Fairly common. Wet locations in muck.

APM-Cly; TRT-Dys, Hct, Sno, Stn.

Schizachne purpurascens (Torrey) Swallen

False medic grass

Common. Rich woodlands and rocky wooded slopes.

TRT-Crd, Mnd, Stn.

Secale cereale $\mathrm{L}$.

Uncommon. Roadside plantings. (+)

TRT-Sno (several sites).

Setaria glauca (L.) P. Beauv.

Yellow foxtail

Rare. One location. Around abandoned railway tracks. (+)

TRT-Dys.

Setaria viridis (L.) P. Beauv.

Green foxtail

Common. Dry sandy roadsides and clearings. $(+)$

TRT_Crd, Dys, Hin, Shb, Stn.

Spartina pectinata Link

Tall cord grass

Rare. One location. Wet shoreline.

TRT-Shb. 
Rare. One location. Roadside ditch. (+)

TRT-Stn.

CYPERACEAE

SEDGE FAMILY

Carex aquatilis Wahlenb.

Common. Marshy and boggy lake edges, in water or on shore.

Sedge

DFB-Lwr; TRT-Liv, Shb.

\section{Carex arctata Boott}

Sedge

Common. Roadside ditches and open woods; on moist or wet sandy soils.

APM-Cly, Ngt; TRT-Crd, Gfd, Hct, Mnd, Shb, Stn.

\section{Carex aurea Nutt.}

Rare. One location. Wet meadow over calcareous substratum.

Sedge

TRT-Mnd.

Carex bebbii (L. Bailey) Olney ex Fern.

Sedge

Common. Wet edges of swamps and lakes.

TRT-Lut, Shb; S.R.-Mnd.

Carex bromoides Schk. ex Willd.

Sedge

Rare. One location. Moist woods.

TRTE-Ans.

Carex brunnescens (Pers.) Poiret ex Lam.

Sedge

Common. On wet ground, in swampy areas and woods.

TRT_Liv, McC, Mnd, Shb; S.R.-Dys.

Carex buxbaumii Wahlenb.

Sedge

Uncommon. Rock crevices and rocky shorelines.

TRT-Dud, McC, Mnd.

Carex canescens $\mathrm{L}$.

Sedge

var. disjuncta Fern.

Rare. One location. Swampy river's edge.

TRT-Stn.

var. subloliacea (Laest.) Hartman

Common. Swamps and wet shores.

TRT -Crd, Dys, Gfd, McC, Mnd; S.R.-Mon.

Carex communis L. Bailey

Sedge

Common. Uplands and shorelines, on dry or moist soils.

APM-Ngt; TRT - Dys, Het, Liv, Mnd, Shb, Stn. 
Carex crawfordii Fern.

Sedge

Common. Moist sandy soils bordering swamps, ponds, and woods.

CAN-Crd; TRT -Dys, Mnd, Shb.

Carex crinita Lam.

Sedge

Fairly common. Wet shores and swampy areas.

TRT-Hct, Shb; S.R.-Hin.

Carex cristatella Britton

Sedge

Rare. One location. Roadside gravel beside stream.

TRT-Hin.

Carex cryptolepis Mackenzie

Sedge

Fairly common. Wet shores of lakes and streams, usually on sandy soil.

APM-Ngt; TRT - Hbn, Hct, Hin, Lwr, Mnd.

Carex cumulata (L. Bailey) Fern.

Sedge

Rare. One location. Damp roadside with dense growth of grasses.

TRT-Lut.

Carex debilis Michaux

Sedge

var. rudgei L. Bailey

Fairly common. Moist woods on humus.

TRT-Hin, Sno.

Carex deflexa Hornem.

Sedge

Rare. One location. Clearing on edge of woods.

TRT-Stn.

Carex deweyana Schwein.

Common. Moist open areas, usually adjacent to woods.

Sedge

APM-Brt; TRT - Crd, Hct, Mnd, Stn.

Carex diandra Schrank

Sedge

Uncommon. Swampy areas, in calcareous regions.

TRT-Crd, Lut, Mnd.

Carex disperma Dewey

Sedge

Common. Swampy edges of woods, and shores.

APM-Cly; TRT-Crd, Dud, Hct, Mnd, Shb.

Carex eburnea Boott

Sedge

Rare. One location. Base of steep cliff of calcareous rock.

TRT-Crd. 
C. cephalantha (L. Bailey) E. Bickn.

C. angustior Mackenzie

C. muricata $\mathrm{L}$.

Common. Rocky, sandy, or marshy lake and stream shores, and wet meadows.

CAN-Crd; DAO-Hct; TRT-Dud, Gfd, Hin, Mnd, Stn.

\section{Carex flava $\mathrm{L}$.}

Sedge

Common. Wet meadows and wet bush roads.

TRT -Crd, Dys, Lut, Mnd.

\section{Carex folliculata $\mathbf{L}$.}

Sedge

Rare. One location. Shallow water off rocky shore.

TRT-Shb.

\section{Carex garberi Fern.}

Sedge

Rare. One location. Wet meadow, in calcareous region.

TRT-Mnd.

Carex gracillima Schwein.

Sedge

Common. Wet open or wooded areas.

TRT -Crd, Lut, Mnd, Stn.

Carex granularis Muhlenb. ex Willd.

Sedge

Rare. One location. Wet open edge of small stream, in calcareous region.

TRT-Mnd.

Carex gynandra Schwein.

Common. On damp or wet edges of woods, on shores, and in sandy

Sedge ditches.

TRT-Cly, Crd, Hct, McC, Mnd, Shb, Stn.

Carex houghtoniana Torrey ex Dewey

Sedge

Fairly common. Open or scrubby, dry or moist, sandy or gravelly locations.

TRT-Crd, Dys, Hct, Ngt, Stn.

Carex hystericina Muhlenb. ex Willd.

Common. On moist or wet shores of lakes, and in damp ditches.

Sedge

TRT -Crd, Shb, Sno.

\section{Carex interior L. Bailey}

Common. Bogs, swamps, wet hollows, and shores; open or wooded.

CAN-Crd; TRT-Dys, Mnd, Mon. 
Carex intumescens Rudge

Sedge

Common. Wet shores and banks of lakes and streams, and damp edges of woods.

APM-Brt; TRT - Dys, Gfd, Hct, Mnd, Shb, Stn.

Carex lacustris Willd.

Uncommon. Grassy stream margins, and lake edges; in water up to

Sedge

$10 \mathrm{~cm}$ deep.

TRT-Dys, Liv.

Carex lanuginosa Michaux

Sedge

Rare. One location. Clearing in moist woods.

TRT-Mnd.

Carex lasiocarpa Ehrh.

Sedge

Common. Bogs, marshes, and wet stream banks.

TRT_Dud, Dys, Hin, Liv, Mnd.

\section{Carex lenticularis Michaux}

Sedge

Uncommon. Wet sand flats on shorelines.

TRT-Hav, Hin, Sno.

Carex leptalea Wahlenb.

Sedge

Uncommon. Moist ground in open disturbed areas, and damp slopes.

TRT - Crd, Mnd.

Carex leptonervia (Fern.) Fern.

Fairly common. Damp deciduous or mixed woods and edges of open

wet areas.

TRT-Crd, Dys, Hct, Shb; S.R._Lut, Mon.

Carex lucorum Willd. ex Link

Sedge

Rare. Two locations. Sandy edge of woods and thin sandy soil over granitic rock.

TRTE-Lut, Stn.

Carex lupulina Muhlenb. ex Willd.

Sedge

Uncommon. Wet depressions in woods.

TRT -3 Hct.

Carex lurida Wahlenb.

Sedge

Fairly common. Wet margins of shores, and damp ditches.

CAN-Shb; TRT-Hin, Shb, Sno; S.R.-Stn. 
Rare. Two locations. Open, dry grassy roadside and old, dry field. TRT-Gfd, Mnd.

Carex michauxiana Boeckeler

Rare. One location. Damp roadside.

Sedge

TRT-Shb.

Carex oligosperma Michaux

Uncommon. Bogs and marshy stream edges.

Sedge

TRT-Hin, Lwr, Shb.

Carex ormostachya Wieg.

Sedge

Common. Open sand-and-gravel flats, fields, and occasionally damp woods.

TRT-Crd, Hct, Hin, Lut, Shb, Stn.

Carex pallescens $\mathbf{L}$.

Uncommon. Damp sandy ditches and open waste sites.

Sedge APM-Cly; TRT-Dys, Sno.

Carex pauciflora Light.

Sedge

Rare. Two locations. Bogs.

CAN-Crd; TRT-Dys.

Carex paupercula Michaux

Rare. Two locations. Swamp edge and bog.

Sedge

TRT-Hct, Mnd.

Carex peckii Howe

Sedge

Common. Open banks, edges of woods, rock depressions, and bogs.

APM-Brt; TRT-McC, Mnd, Stn; S.R.-Dys, Hbn.

Carex pedunculata Muhlenb. ex Willd.

Sedge

Uncommon. Open or wooded lakeshores.

APM-Brt; TRT - Mnd.

Carex pensylvanica Lam.

Sedge

Uncommon. Open, mossy sand flats and rocky grassy slopes.

TRT-Dud, Dys.

Carex plantaginea Lam.

Sedge

Rare. Two locations. Sugar maple forest and moist coniferous woods.

TRT-Dys, Hct. 
Rare. One location. Sugar maple forest, on rich humus.

$\mathrm{CAN}-\mathrm{Crd}$.

Carex projecta Mackenzie

Uncommon. Damp or wet depressions in woods and meadows.

Sedge

TRT-Gfd, Hct.

Carex pseudo-cyperus $\mathrm{L}$.

Sedge

Common. Swamps, wet woods, and wet shores.

TRT-Crd, Hct, Lut, Sno, Stn; S.R.-Mnd.

Carex radiata (Wahlenb.) Small

C. rosea auct. non Willd. (see Webber and Ball, 1984)

Sedge

Uncommon. Roadsides, old fields, edges of woods, and grassy banks.

TRT-Crd, Glm, Hct, Mnd.

Carex retrorsa Schwein.

Common. Wet ditches, swamp edges, and wet edges of woods.

Sedge

TRT -Crd, Dys, Hct, Lut.

\section{Carex rugosperma Mackenzie}

Uncommon. Mixed woods and sand flats.

Sedge

TRT-Mnd, Shb; S.R.-Hbn.

Carex scabrata Schwein.

Sedge

Fairly common. Damp or wet woods.

TRT-Dys, Hbn, Hct; TRTE-Ans.

Carex scoparia Schk. ex Willd.

Sedge

Common. Wet sandy shores of lakes, ponds, and streams.

TRT-Crd, Dys, Glm, Hct, Hin, Stn; S.R.-Sno.

\section{Carex sprengelii Dewey ex Sprengel}

Sedge

Uncommon. Dry open ditches and open woodlands.

TRT-Crd, Hct, Mnd; S.R.-Dys.

Carex stipata Muhlenb. ex Willd.

Common. Moist to wet banks, roadsides, and marsh edges.

Sedge

TRT -Crd, Gfd, Hct, Mnd.

Carex stricta Lam.

Sedge

Common. Open wet locations: swamps, marshes, ditches, and meadows.

APM-Cly; TRT-Crd, Dud, Dys, Liv, Stn; S.R.-Shb. 
Uncommon. Moist open edges of woods, stream banks, and damp meadows.

TRT-Lut, Sno, Stn; S.R.-Mnd.

\section{Carex trisperma Dewey}

Common. Bogs, marshy lake edges, and wet wooded roadsides.

APM-Cly; TRT_-Dud, Dys, Hct, Shb; S.R.-Liv.

\section{Carex tuckermanii Dewey}

Rare. One location. Damp edge of coniferous woods.

TRT-Dys.

\section{Carex utriculata Boott}

C. rostrata auct. non Stokes

Sedge

Common. Edges of lakes, stream banks, and marshes.

TRT_Dud, Dys, Hbn, Hct, Shb; S.R.-Liv.

Carex vesicaria $\mathrm{L}$.

Sedge

Uncommon. Shallow water and wet borders of lakes and bogs.

APM-Cly; TRT -Crd, Gfd, Mnd, Stn.

Carex viridula Michaux

Sedge

Rare. One location. Edges of old, mostly dried up cat-tail marsh.

TRT-Crd.

Carex vulpinoidea Michaux

Rare. Two locations. Damp, open lake bank and wet meadow.

Sedge

TRT-Dys, Sno.

Cladium mariscoides (Muhlenb.) Torrey

Twig-rush

Fairly common. Sphagnum mats on boggy shores.

TRT-Dys, Hin, McC, Shb; S.R.-Crd.

Cyperus diandrus Torrey

Galingale

Rare. One location. Sandy lakeshore.

TRT-Stn.

Cyperus rivularis Kunth

Rare. One location. Sandy shore of river.

Umbrella sedge

TRT-Sno. 
Common. Wet shores, and shallow water of lakes and ponds, on sand or mud; often in dense colonies.

TRT-Crd, Dys, Gfd, Hbn, Hct, Mnd, Shb; S.R.-Hin.

Eleocharis acicularis (L.) Roemer and Schultes

Needle rush

Common. Sandy or mucky shores of lakes and streams; growing in mats.

APM-Cly; DAO-Crd, McC; TRT-Dys, Gfd, Hct, Mnd, Shb;

S.R.-Glm.

\section{Eleocharis intermedia Schultes}

Spike-rush

Uncommon. Mucky or muddy shores of streams and lakes.

TRT-McC, Stn.

Eleocharis obtusa (Willd.) Schultes

Spike-rush Common. Sandy or muddy shores and flats, and swampy areas; growing in clumps.

TRT-Crd, Dys, Hbn, Hct, Mnd, Shb.

Eleocharis olivacea Torrey

Spike-rush

Uncommon. Boggy and marshy shores and mucky edges of streams.

TRT-Crd, Dys, Shb.

Eleocharis ovata (Roth) Roemer and Schultes

Spike-rush

Uncommon. Wet sand or mud flats, edges of streams, and various other wet places.

TRT-Crd, Glm, Hbn, Stn.

Eleocharis palustris (L.) Roemer and Schultes

E. smallii Britton

Spike-rush

Fairly common. Shallow water and marshy edges of streams and lakes.

DAO-Crd, Dys; TRT-Crd, Dys, Hct, Mnd.

Eleocharis pauciflora (Light.) Link

Spike-rush var. fernaldii Svenson

Rare. One location. Shore of lake, on mud flat.

TRT-Dys.

Eleocharis robbinsii Oakes

Spike-rush

Uncommon. Lakes and streams, in water up to $30 \mathrm{~cm}$ deep, on soft mud; leaves limp and floating.

TRT_Dys, McC, Shb; S.R.-Hin. 
Uncommon. Bogs and swamps.

TRT-Dys, Hct; S.R.-Mon.

Eriophorum virginicum $\mathbf{L}$.

Tawny bog-cotton

Common. Sphagnum bogs, and occasionally wet ditches.

CAN-Hin; TRT-Cly, Dys, Hct, Hin, McC; S.R.-Shb.

Eriophorum viridi-carinatum (Engelm.) Fern.

Cotton-grass

Rare. One location. Damp sedge meadow, in calcareous region; 20 to 25 scattered plants.

TRT-Mnd.

Rhynchospora alba (L.) M. Vahl Beak rush

Common. Bogs and boggy shores of lakes.

DFB-Lwr; TRT-Dys, Hbn, Liv, McC; S.R.-Hin, Shb.

Rhynchospora capitellata (Michaux) M. Vahl

Beak rush

Uncommon. Damp sand flats and lakeshores.

TRT-Hbn, Hin, Shb.

Rhynchospora fusca (L.) Aiton f.

Beak rush

Uncommon. Bog mats and boggy shores.

CAN-Crd; TRT-Hin, Lwr, McC.

Scirpus acutus Muhlenb. ex Bigelow

Hardstem bulrush

Uncommon. Shallow water near edges of lakes, on sandy soil.

TRT-Dys, Hct, Mnd; S.R.-Lut.

Scirpus atrovirens Willd.

Bulrush

Common. Wet depressions, stream banks, lakeshores, and reed marshes.

TRT-Cly, Het, Mnd, Lut.

Scirpus cyperinus (L.) Kunth

Wool-grass

Common. Damp roadsides, shorelines, and marshes.

TRT-Cly, Crd, Hbn, Hct; S.R.-Lut.

Scirpus georgianus Harper

Bulrush

Rare. One location. Roadside overlooking Lake Kashagawigamog.

TRT-Dys.

Scirpus microcarpus Presl

Bulrush

var. rubrotinctus (Fern.) Jones

Rare. Two locations. Edges of swamps.

TRT-Hbn, Stn. 
Scirpus pendulus Muhlenb. ex Willd.

Bulrush

S. lineatus Michaux

Rare. One location. Bottom land of drained beaver pond.

TRT-Mnd.

Scirpus subterminalis Torrey

Water bulrush

Uncommon. Slow river currents and backwaters to depths of $30 \mathrm{~cm}$ or more; leaves limp and floating.

TRT-Dys, 2 Stn.

Scirpus validus M. Vahl

Soft-stem bulrush

var. creber Fern.

Common. Rivers up to 30 to $40 \mathrm{~cm}$ deep, swampy lake edges, and marshes; large colonies in marshes.

TRT-Hbn; S.R.-Crd, Dys, Lut.

ARACEAE

ARUM FAMILY

Acorus americanus (Raf.) Raf.

Sweetflag

Rare. Three locations, including sandy delta and swampy lake edge; small colonies growing with sedges.

TRT -2 Mnd, Stn.

Arisaema triphyllum (L.) Schott

Jack-in-the-pulpit

Common. Damp or wet locations in, or on the edges of, woods.

TRT_Gfd, Hav, Hct, Mnd, Stn; S.R.-Brt, Dys.

\section{Calla palustris $\mathrm{L}$.}

Wild calla, Water-arum

Common. Wet, low edges of streams, ponds, and marshes. Dense colonies spreading by stout underwater rhizomes.

TRT-Crd, Dud, Gfd, Hbn, Hct; S.R.-Ans, Hin.

\section{LEMNACEAE}

DUCKWEED FAMILY

\section{Lemna minor $\mathrm{L}$.}

Duckweed

Common. Pools, swamps, and edges of quiet streams; sometimes covering entire surfaces of ponds in swamps.

TRT-Hbn; S.R._Lut, Mon, Shb.

Spirodela polyrhiza (L.) Schleiden

Greater duckweed

Uncommon. Growing with Lemna minor, or alone in similar habitats.

TRT-Crd, Dys, Hbn.

XYRIDACEAE

Xyris difformis Chapman

YELLOW-EYED GRASS FAMILY

Yellow-eyed grass

Uncommon. Sphagnum bogs, and a sand-and-gravel lakeshore. Rare in

Ontario (Argus et al., 1982-1987).

TRT -2 Hin, Shb. 
Uncommon. Boggy edges of streams, ponds, and small lakes, and occasionally wet sandy shores.

TRT-_Crd, Gfd, Hin, McC, Shb.

\section{ERIOCAULACEAE}

PIPEWORT FAMILY

Eriocaulon septangulare With.

Pipewort

Common. Edges of ponds and small lakes in water up to $150 \mathrm{~cm}$ deep, and wet shores.

TRT-Cly, Dys, Gfd, Hbn, Hct, Hin, Mnd, Shb.

\section{PONTEDERIACEAE}

PICKEREL-WEED FAMILY

Pontederia cordata $\mathrm{L}$.

Common. Shallow water along shores of lakes and rivers; usually in large dense colonies.

TRT-Crd, Hct, Mnd, Shb; S.R.-Hin, Sno.

\section{JUNCACEAE}

Juncus acuminatus Michaux

RUSH FAMILY

Rush

Rare. Two locations. Sandbar and swampy lake edge. Rare in Ontario

(Argus et al., 1982-1987).

TRT-Mnd; S.R.-Shb.

Juncus articulatus $\mathbf{L}$.

Rush

Uncommon. Edges of lakes and cat-tail marshes, on wet sand or sandy muck.

TRT-Crd, Dys, Lut.

Juncus brachycephalus (Engelm.) Buchenau

Rush

Rare. One location. Wet ditch, on sand and gravel.

TRT-Stn.

Juncus brevicaudatus (Engelm.) Fern.

Common. Wet sandy ditches and sandy shores.

DFB-Lwr; TRT-Cly, Crd, Dys, Hbn, Hct, Mnd, Shb.

Juncus bufonius L.

Toad rush

Common. Lakeshores and other open, flat, wet or damp sandy areas.

TRT-Crd, Dys, Gfd, Hbn.

Juncus canadensis J. Gay ex Laharpe

Canada rush

Common. Marshy and boggy shores, and quiet backwaters.

TRT-Dys, Gfd, Hin, Stn; S.R.-Crd, Lut, Shb. 
Rare. Two locations. Moist, open grassy area in park, and damp weedy area beside abandoned railroad.

TRT-Dys, Gfd.

\section{Juncus effusus $\mathbf{L}$.}

Common. Edges of streams and lakeshores, wet ditches, and open, damp sand flats; growing in or out of water.

TRT-Cly, Crd, Dys, Gfd, Hct, Mnd, Shb, Sno.

\section{Juncus filiformis $\mathbf{L}$.}

Rush

Common. Sandy edges of lakes, bays, and streams.

TRT_Crd, Hbn, Hin, Liv, Mnd; S.R.-Dys.

Juncus militaris Bigelow

Bayonet rush

Rare. One location. Shallow water of lake on soft sand and muck bottom; plants up to $135 \mathrm{~cm}$ in height.

TRT-Hin.

\section{Juncus nodosus $\mathbf{L}$.}

Rush

Common. Edges of marshes and shores of streams.

TRT-Crd, Lut, Sno.

\section{Juncus pelocarpus E. Meyer}

Rush

Common. Mucky or muddy shores of lakes and streams.

TRT-Crd, Dys, Hin, McC; S.R.-Mnd, Shb.

Juncus tenuis Willd.

Path rush

Common. Damp or dry, sandy or muddy open flats and banks.

TRT -Crd, Dys, Hct, Mnd.

Luzula multiflora (Retz.) Lej.

L. campestris (L.) DC. var. multiflora (Ehrh.) Čelak.

Wood rush

Common. Open or partly shaded sites, usually on damp soil.

TRT-Dys, Hbn, Sno, Stn.

\section{LILIACEAE}

Allium schoenoprasum $\mathbf{L}$.
LILY FAMILY

Wild chive

Rare. One location. One clump on edge of gravel road; escape from cultivation. (+)

TRT-Stn.

Common. Woods and edges of woods; in dense colonies.

APM-Brt; DFB-McC; TRT-Dud, Hct, Mnd. 
Uncommon. Sandy roadsides and clearings. (+)

TRT-Mnd, Stn; S.R.-Hbn.

Clintonia borealis (Aiton) Raf.

Corn lily

Common. Deciduous or mixed woods, on moist humus.

TRT -Crd, Dud, Gfd, Hbn, Hct, Shb, Stn.

Erythronium americanum Ker Gawler

Trout lily

Common. Deciduous or mixed woods, on moist humus; in large dense colonies, often with a small number of plants bearing flowers.

TRT-Crd, Gfd, Hbn, Hct, Shb; S.R.-Dys, Mnd.

Hemerocallis fulva (L.) L.

Orange day-lily

Uncommon. Damp ditches and grassy banks; escapes from cultivation, but well established. $(+)$

APM-Ngt; TRT-Lut, McC, Stn.

Hemerocallis lilioasphodelus $\mathrm{L}$.

Yellow day-lily

H. flava (L.) L.

Uncommon. Damp roadside ditches; in small dense colonies; escapes from cultivation, but well established. (+)

TRT-Lut.

\section{Lilium philadelphicum L.}

Wood lily

Rare. One location. Moist woods.

$\mathrm{OAC}-\mathrm{Crd}$.

\section{Maianthemum canadense Desf.}

Wild lily-of-the-valley

Common. Moist deciduous woods and mossy banks; in small to large colonies.

TRT-Crd, Dud, Gfd, Hbn, Hct, Mnd, Shb, Stn.

\section{Medeola virginiana $\mathrm{L}$.}

Indian cucumber-root

Fairly common. Deciduous or mixed woods, on leafy humus; locally sparse, with plants occurring singly, often some distance apart.

DFB-Ngt; TRT-Crd, Gfd, Hct, Mnd, Shb; S.R.-Hin.

Polygonatum pubescens (Willd.) Pursh

Hairy Solomon's-seal

Common. Woods and edges of woods.

TRT-Crd, Hav, Hct, Mnd, Stn; TRTE-Ans.

Smilacina racemosa (L.) Desf.

False Solomon's-seal

Common. Open roadsides and edges of woods; in large colonies.

TRT-Gfd, Hct, Mnd; S.R.-Dys, Glm, Lut, Sno. 
Smilacina stellata (L.) Desf.

Starry Solomon's-seal

Uncommon. On low river banks, and beside old railway tracks on sandy soil.

TRT-Crd, Sno; S.R.-Mnd.

Smilacina trifolia (L.) Desf.

Three-leaved Solomon's-seal

Common. Damp or wet, open or partly treed bogs; in sphagnum.

TRT-Dud, Dys, Hct, McC.

Smilax hispida Muhlenb.

Prickly greenbrier

Rare. Two locations. Beside creek and on river bank; climbing on tall shrubs.

TRT-Mnd, Sno.

Streptopus roseus Michaux

Rose twisted-stalk

Common. Moist woods and partly shaded grassy banks.

APM-Ngt; TRT-Gfd, Hct, Liv, Mnd; S.R.-Dys, Hin.

Trillium erectum $\mathrm{L}$.

Red trillium

Common. Moist woods. Widespread in the county. (A few aberrant forms with green patches on petals resulting from disease, TRT-Mnd.)

TRT-Hct, Mnd; S.R.-Dys.

f. luteum Louis-Marie

Red trillium (yellow form)

Rare. Two locations. Roadside, and wet cedar woodland.

DFB-Hct; TRT - Hct.

Trillium grandiflorum (Michaux) Salisb.

White trillium

Common. Rich moist woods; large colonies in southern townships, rare in northern two-thirds of county. (A number of aberrant forms with green streaks on petals resulting from disease, TRT - Lut, Mnd.)

APM-Brt; TRT-Hct, Lut, Mnd, Stn; S.R.-Crd, Dud.

Trillium undulatum Willd.

Painted trillium

Fairly common. Deciduous or mixed woods; more abundant in northern townships.

TRT-Hct, Liv, Mnd; S.R.-Hin.

Uvularia grandiflora Smith

Large-flowered bellwort

Common. Deciduous woods, on leafy humus.

APM-Brt; TRT-Hbn, Hct; S.R.-Crd, Dys, Lut, Mnd. 
Uncommon. Damp grassy flats in partial shade, and low, open grassy banks; escapes from cultivation. $(+)$

TRT-Crd; S.R.-Glm, Stn.

\section{IRIDACEAE}

IRIS FAMILY

Iris versicolor $\mathrm{L}$. Wild iris, Blue flag

Fairly common. Rocky, sandy, or marshy shores, and shallow water. TRT-Crd, Dys, Gfd, Hct; S.R.-Hin, Mon, Stn.

Sisyrinchium montanum E. Greene

Fairly common. Open flat sites and sandy grassy banks.

TRT-Gfd, Hct, Lut, Mnd, Shb; S.R.-Crd.

\section{ORCHIDACEAE}

Calopogon tuberosus (L.) Britton, Sterns, and Pogg.

ORCHID FAMILY

C. pulchellus (Salisb.) R. Br.

Grass-pink

Rare. Two locations. Open sphagnum bogs bordering lakes; about one hundred plants in one of these locations.

TRT -2 Hin.

\section{Coeloglossum viride (L.) Hartman}

Long-bracted green orchid var. virescens (Muhlenb.) Luer

Habenaria viridis (L.) R. Br. var. bracteata (Willd.) A. Gray

Uncommon. Mixed or deciduous woods.

TRT-Hct; S.R.-Brt, Hct.

Corallorhiza maculata (Raf.) Raf.

Spotted coral-root

Uncommon. Damp deciduous or mixed woods, on humus.

TRT-Crd, Dys, Hct, Shb, Stn.

\section{Corallorhiza striata Lindley}

Striped coral-root

Uncommon. Dense, damp maple forests, and mixed woods; on humus.

TRT-Crd, Hct, Mnd.

\section{Corallorhiza trifida Châtel}

Early coral-root

Uncommon. Cedar-balsam swamp and wet mixed woods.

TRT-Mnd; S.R.-Dys.

Cypripedium acaule Aiton

Pink moccasin flower, Pink lady's-slipper

Common. Coniferous or mixed woods; usually in shade, on humus over sand; often numerous and scattered.

TRT-Crd, Dud, Hbn, Hct, McC, Shb; S.R.-Brt, Hin, Liv. 
f. albiflorum Rand and Redf.

White-lipped moccasin flower

Rare. One location. Mixed woods on lake bank; scattered sparsely among forma acaule.

TRT-Liv.

Cypripedium calceolus $\mathrm{L}$.

var. pubescens (Willd.) Correll

Uncommon. Damp mixed woods.

TRT-Lut; S.R._Lut, Sno.

Cypripedium reginae Walter

Large yellow lady's-slipper

Rare. One location. Tamarack-cedar bog.

TRT-Glm.

Epipactis helleborine (L.) Crantz

Helleborine

Common. On roadsides at edges of deciduous or mixed woods, and occasionally in coniferous woods. $(+)$

APM-Ngt; DAO-Hct, Ngt; TRT-Dys, Hct, Mnd; S.R.-Lut.

Goodyera oblongifolia Raf.

Menzies' rattlesnake-plantain

Rare. One location. Old road through woods, on sand and gravel.

TRT-Mnd.

Goodyera repens (L.) R. Br.

Dwarf rattlesnake-plantain

var. ophioides Fern.

Uncommon. Damp or dry coniferous woods.

TRT - Hct, Mnd, Shb.

Goodyera tesselata Lodd.

Rattlesnake-plantain

Common. Mixed woods, dry uplands, and wet lowlands in sphagnum moss.

APM-Lwr; DFB_Lwr; TRT -Dys, Gfd, Hbn, Hct, Hin, Shb.

Liparis loeselii (L.) L. C. M. Richard

Loesel's twayblade

Rare. One location. Along lakeshore in sand and gravel of abandoned road. TRT-Stn.

Listera auriculata Wieg.

Auricled twayblade

Rare. One location. Shaded, damp sand-and-clay bank of river under alders and balsam trees. About ten scattered plants, a few in moss; the most southern Canadian site recorded in Ontario for this species (Whiting and Catling, 1977).

TRT-Sno. 
Rare. One location. Cedar-balsam swamp, in calcareous region; 20 to 30 plants in one area of swamp.

TRT-Mnd.

Malaxis unifolia Michaux

Green adder's-mouth

Uncommon. Mixed woods and rocky or sandy open sites.

TRT -Crd, Hbn, Mnd, Shb; S.R.-Dys, Hin.

Platanthera clavellata (Michaux) Luer

Club-spur orchid

Habenaria clavellata (Michaux) Sprengel

Common. Open, flat sandy sites with low vegetation of sedges and mosses, and woods near edges of lakes and bogs; often locally abundant.

TRT-Dud, Hbn, Hin, McC, Shb; S.R.-Dys.

Platanthera hyperborea (L.) Lindley

Tall northern green orchid Habenaria hyperborea (L.) R. Br.

Fairly common. Shaded, wet areas: ditches, swamps, and hollows in woods; usually in muck.

TRT-Crd, Hct, Mnd, Stn.

Platanthera lacera (Michaux) G. Don

Green fringed orchid

Habenaria lacera (Michaux) Lodd.

Uncommon. Damp ditches and moist meadows.

TRT_-Mnd, Sno; S.R.-Dys.

Platanthera obtusata (Banks ex Pursh) Lindley

Blunt-leaf orchid

Habenaria obtusata (Banks ex Pursh) Richardson

Uncommon. Damp woods and edges of bogs.

TRT-Dud, Hct, Mnd.

Platanthera orbiculata (Pursh) Lindley

Large round-leaved orchid var. orbiculata

Habenaria orbiculata (Pursh) Torrey

Uncommon. Coniferous or mixed woods, on rich moist soils.

TRT-Hct, Stn; S.R.-Dys.

var. macrophylla (Goldie) Luer

Habenaria orbiculata (Pursh) Torrey var. macrophylla (Goldie) Boivin

Rare. Two locations. Moist woods at edge of cedar-balsam swamp, and wet woods. Rare in Ontario (Argus et al., 1982-1987).

CAN-Mnd; PENN-Stn; TRT-Mnd, Stn (photostat of PENN specimen). 
Platanthera psycodes (L.) Lindley

Purple fringed orchid

Habenaria psycodes (L.) Sprengel

Rare. Two locations. Wet bank, and rich soil on small island in stream.

TRT-Hbn, Hct.

Pogonia ophioglossoides (L.) Juss.

Rose-pogonia

Uncommon. Bogs and boggy shores.

REW-Crd; TRT-Crd, Dys, McC; S.R.-Hin.

Spiranthes casei Catling and Cruise

Case's ladies' tresses

Fairly common. Sand-and-gravel roadsides, roadside banks, and open grassy fields.

TRT-Dys, Shb, Stn; WAT-Hin.

Spiranthes cernua (L.) L. C. M. Richard

Nodding ladies' tresses

Common. Damp ditches, moist meadows, and sand-and-gravel flats

with mosses.

TRT-Crd, Dys, Hbn, Hct, Hin, Mnd, Shb, Sno.

Spiranthes lacera (Raf.) Raf.

Slender ladies' tresses

Uncommon. Rock outcrops and wooded roadsides.

REW-Hin, Shb; TRT-Hbn, Hct, Hin, Mnd, Shb.

Spiranthes romanzoffiana Cham.

Hooded ladies' tresses

Uncommon. Damp or dry roadsides, depressions on rocky banks, and grassy flats on sandy soil.

TRT-Hbn, Hct, Mnd; S.R.-Dys, Hin.

Spiranthes lacera (Raf.) Raf.

Hybrid ladies' tresses

$\times$ S. romanzoffiana Cham.

Rare. One location. Shallow depression on rocky roadside. Unusual hybrid with two locations recorded in Ontario, one in the Parry Sound District and the other in Haliburton (Whiting and Catling, 1986).

DAO-Hin; TRT-Hin.

\section{DICOTYLEDONS}

SALICACEAE

Populus alba L.

WILLOW FAMILY Uncommon. Fields and roadsilver poplar spreading. (+)

TRT-Dys, Mnd, Stn. 
Common. Sand flats, roadside banks, and occasionally rocky ridges; often in tall stands.

TRT_Gfd, Mnd; S.R.-Crd, Dud, Lut.

\section{Populus grandidentata Michaux}

Large-toothed aspen

Common. Tall mixed woods or large pure stands; on leaf-covered sandy soil.

TRT-Dys, Hct; S.R._Ans, Hin, Mnd, Sno, Stn.

\section{Populus tremuloides Michaux}

Quaking aspen

Common. Pure stands, mixed woods, and edges of meadows and woods; on sandy soils.

TRT-Crd, Dys, Gfd, Hct, Stn.

Salix bebbiana Sarg.

Beaked willow

Common. Moist to very wet areas-thickets, fields, and swamps-and sandy banks.

TRT-Hbn, Hct, Mnd; S.R.-Hin, Lut.

Salix discolor Muhlenb.

Pussy willow

Common. Dry sandy areas and wet swampy sites; often with various shrub species.

TRT-Hct, Mnd, Shb; S.R.-Crd, Hct, Hin.

Salix eriocephala Michaux

Stiff willow

$S$. rigida Muhlenb.

Rare. One location. Four shrubs in roadside thicket, on damp sandy soil.

TRT -Stn.

Salix fragilis $\mathrm{L}$.

Crack willow

Common. River and lake banks, lawns, and damp roadsides; on clay or sand; branches very brittle; introduced and well established. $(+)$

APM-Ngt; TRT - Dys, Mnd.

Salix humilis Marshall

Upland willow

Common. Various open, dry or damp areas.

TRT-Dys, Hct, Mnd; S.R.-Sno.

Salix lucida Muhlenb.

Shining willow

Common. Open, damp to wet areas, with other species of willows and shrubs.

TRT-Dys, Hbn, Hct; S.R.-Crd. 
Common. Wet roadside thickets, swamps, bogs, and damp fields; usually in dense colonies.

TRT - Dys, Hbn, Hct, Mnd, Sno; S.R.-Stn.

\section{Salix planifolia Pursh}

Flat-leaved willow

Rare. One location; three shrubs. Open, damp roadside ditch.

TRT-Mnd.

Salix pyrifolia Andersson

Balsam willow

Fairly common. Edges of marshes, bogs, and lakes, and wet meadows.

APM-Cly; TRT-Dys, Hin; S.R.-Stn.

\section{MYRICACEAE}

BAYBERRY FAMILY

Comptonia peregrina (L.) Coulter

Sweet-fern

Myrica asplenifolia auct. non L.

Uncommon. Dry sandy areas and damp shores; in colonies.

TRT-Dys, Gfd, Shb; S.R.-Sno.

\section{Myrica gale L.}

Sweet gale

Common. Shores, sphagnum flats, and wet banks; in large colonies.

TRT-Dys, Hct, Hin, Shb; S.R.-Crd.

BETULACEAE

Alnus rugosa (Du Roi) Sprengel

BIRCH FAMILY

Common. Swamps, and edges of ponds, streams, and lakes; widespread in county and usually in large colonies.

TRT-Crd, Dud, Hct, Mnd, Shb; S.R.-Glm, Mon.

\section{Betula alleghaniensis Britton}

Yellow birch

$B$. lutea Michaux $\mathrm{f}$.

Common. Tall mixed woods.

TRT_-Gfd, Mnd; S.R.-Dys, Hin, Sno.

Betula papyrifera Marshall

White birch, Paper birch

Common. Mixed woods on humus over granite or sand; usually in colonies.

TRT_-Gfd, Hbn, Mnd; S.R.-Dys, Hin, Lut.

Corylus cornuta Marshall

Beaked hazel

Common. Edges of banks, streams, and woods.

TRT-Brt, Hbn, Hct, Mnd. 
Common. Mixed woods and woodlands.

TRT-Hct, Mnd, Stn; S.R.-Dys, Lut.

FAGACEAE

Fagus grandifolia Ehrh.

BEECH FAMILY

Common. Deciduous or mixed woods, on rich humus.

American beech

TRT-Hct, Mnd, Shb; S.R.-Brt, Lut.

Quercus rubra L.

Red oak

Q. borealis Michaux f.

Common. Rocky ridges and mixed woods; often on thin soil.

APM-Ngt; TRT-Dud, Mnd, Shb, Stn.

ULMACEAE

ELM FAMILY

Ulmus americana $\mathrm{L}$.

White elm

Uncommon. At edges of woods and in fields; in groves of mixed deciduous trees.

TRT-Dys, Mnd, Stn.

Ulmus thomasii Sarg.

Rock elm

Rare. One location. Two trees at edge of woods on damp roadside.

TRT-Dys.

MORACEAE

Cannabis sativa $\mathrm{L}$.

MULBERRY FAMILY

Rare. One location. Waste ground. (+)

APM-Ngt.

Humulus lupulus L.

Common hop

Uncommon. Dry gravelly roadsides. (+)

TRT - Hct, Mnd.

URTICACEAE

NETTLE FAMILY

Boehmeria cylindrica (L.) Sw.

False nettle

Rare. One location. Mixed woods. Large colony surrounding stagnant pool.

TRT-Lut.

Laportea canadensis ( $\mathrm{L}$.$) Wedd.$

Wood nettle

Common. Low, wet swampy areas and damp edges of woods.

TRT-Crd, Hct, Mnd, Sno. 
Uncommon. Deciduous woods, and edges of woods on damp roadsides.

TRT-Dys, Hct.

\section{SANTALACEAE}

Comandra umbellata (L.) Nutt.

C. richardsiana Fern.

Uncommon. Rock outcrops and lakeshore rock ledges.

TRT-Lut; S.R.-Hin, Shb.

\section{POLYGONACEAE}

\section{Polygonum amphibium L.}

BUCKWHEAT FAMILY

$P$. coccineum Muhlenb.

P. natans Eaton

Common. Quiet waters up to $1 \mathrm{~m}$ deep, and edges of sandy or muddy shores.

TRT -Crd, Dys, Hbn, Hct, Hin, Lut, Mnd, Shb, Stn; S.R. - Mon.

\section{Polygonum aviculare $\mathrm{L}$.}

Prostrate knotweed

Fairly common. Dry, sandy, barren areas and sparse lawns. (+)

TRT-Dys, Stn; S.R.-Hin, Mnd.

\section{Polygonum careyi Olney}

Bristly smartweed

Rare. Open banks and wet shores; singly or in colonies. Rare in Ontario

(Argus et al., 1982-1987).

TRT -Gfd, Hbn, Stn.

\section{Polygonum cilinode Michaux}

Bindweed

Common. Dry roadsides, open woodlands, and sandy shores.

TRT-Crd, Gfd, Hct, Mnd, Shb.

Polygonum convolvulus $\mathrm{L}$.

Black bindweed

Fairly common. Dry sandy roadsides and wastelands. (+)

TRT-Cly, Dys, Gfd, Mnd.

Polygonum cuspidatum SieboId and Zucc.

Japanese knotweed

Rare. One location. Damp roadside; in small colony. (+)

TRT-Dys.

\section{Polygonum hydropiper L.}

Common smartweed

Fairly common. Wet ditches and edges of swamps and streams. (+)

TRT-Hct, Mnd, Sno, Stn. 
Uncommon. Sand flats and other open areas.

TRT-Gfd, Hbn.

Polygonum persicaria $\mathrm{L}$.

Lady's-thumb

Common. Waste areas and roadsides. (+)

TRT-Crd, Hbn, Hct; S.R.-Lut.

\section{Polygonum punctatum Elliott}

Dotted smartweed

Common. Wet or damp stream edges.

TRT-Dud, Glm, Hbn, Hct, Hin, Mnd, Stn.

Polygonum sagittatum L.

Tear-thumb

Common. Dry or damp ditches, and edges of ponds and swamps.

TRT-Crd, Gfd, Hct, Hin; S.R.-Mnd.

Rumex acetosella $\mathrm{L}$.

Sheep-sorrel

Common. Sand flats, ditches, and damp lakeshores. (+)

APM-Ngt; TRT-Crd, Hct, Mnd; S.R.-Hin.

Rumex crispus $\mathbf{L}$.

Sour dock, Curly dock

Uncommon. Dry or damp grassy roadsides. (+)

TRT-Dys, Stn; S.R.-Hin.

Rumex obtusifolius L.

Bitter dock

Fairly common. Lake edges, and ditches among sedges and grasses. $(+)$

APM-Lwr; TRT-Dys, Hct, McC, Mnd.

Rumex patientia $\mathbf{L}$.

Patience dock

Rare. One location. Grassy clearing beside lake. (+)

TRT-Dys.

\section{CHENOPODIACEAE}

GOOSEFOOT FAMILY

Chenopodium album $\mathrm{L}$.

Lamb's quarters

Fairly common. Sandy roadsides and open disturbed ground. (+)

TRT-Hct, Mnd; S.R.-Mnd.

Chenopodium capitatum (L.) Ascherson

Strawberry-blite

Uncommon. Damp banks, and clearings with shrubs or grasses; in small colonies.

APM-Cly; TRT -Dud, Hct, Mnd, Stn.

Chenopodium hybridum $\mathrm{L}$.

Maple-leaved goosefoot

Uncommon. Disturbed sites on sandy soil.

TRT - Dys, Hbn, Hct. 
Rare. One location. On rock ballast of abandoned railroad tracks; in small colony. $(+)$

TRT-Sno.

AMARANTHACEAE

AMARANTH FAMILY

Amaranthus retroflexus $\mathrm{L}$.

Pigweed

Uncommon. Disturbed waste areas. (+)

TRT-Dys; S.R.-Crd, Dys.

\section{NYCTAGINACEAE}

Oxybaphus nyctagineus (Michaux) Sweet

FOUR-O'CLOCK FAMILY

Mirabilis nyctaginea (Michaux) MacMill.

Umbrellawort

Rare. Two locations. Large colony in low damp area along abandoned railroad tracks, and two plants among trees at the edge of meadow. (+)

TRT-Dys; S.R.-Lut.

\section{AIZOACEAE}

CARPETWEED FAMILY

Mollugo verticillata $\mathrm{L}$.

Carpetweed

Rare. Two locations. Open sand-and-gravel sites, in large spreading patches. (+)

APM-Ngt; TRT-Mon.

PORTULACACEAE

Claytonia caroliniana Michaux

PURSLANE FAMILY

Spring-beauty

Common. Damp woods, on humus.

TRT - Hbn, Hct, Mnd.

CARYOPHYLLACEAE

Arenaria serpyllifolia $\mathbf{L}$.

PINK FAMILY

Uncommon. Soil pockets on rocky slopes and dry sandy sites. (+)

TRT_Lut, Mnd, Shb, Stn.

Cerastium arvense $\mathbf{L}$.

Field chickweed

Rare. One location. Open grassy slope.

TRT-Dys.

Cerastium fontanum Baumg.

Mouse-ear chickweed subsp. triviale (Link) Jalas

Common. Grassy or sandy, open or partly shaded areas. (+)

TRT-Gfd, Hct, Mnd, Shb, Stn.

Dianthus armeria L.

Deptford pink

Uncommon. Sandy roadsides among grasses. (+)

TRT-Mnd, Stn. 
Rare. Two locations. Dry sandy flat and open hillside; escapes from cultivation. (+)

TRT-Lut, Stn.

Dianthus plumarius $\mathbf{L}$.

Garden pink

Rare. One location. Edge of woods; escape from cultivation. (+)

TRT-Stn.

Herniaria glabra $\mathrm{L}$.

Knawel

Rare. One location. In cracks of pavement of old road. (+)

TRT-Mnd.

Lychnis chalcedonica $\mathrm{L}$.

Scarlet lychnis

Rare. One location. Edge of poplar woods among shrubs and tall herbs;

escape from culitvation. $(+)$

TRT-Mnd.

Sagina procumbens $\mathbf{L}$.

Pearlwort

Uncommon. Two locations. Cracked pavement and gravel flats. (+)

TRT -2 Mnd; S.R.-Glm.

Saponaria officinalis $\mathbf{L}$.

Bouncing-bet

Common. Roadsides, clearings, and damp gravel pits. (+)

TRT - Hin, Shb.

Silene antirrhina $\mathrm{L}$.

Sleepy catchfly

Uncommon. Sandy areas, and abandoned railroad tracks in cinders.

TRT--Dys; S.R.-Sno.

Silene armeria $\mathbf{L}$.

Garden catchfly

Uncommon. Edges of woods and soil pockets on rocks; escapes from

cultivation. $(+)$

APM-Ngt; TRT-Hbn, Hct.

Silene noctiflora $\mathbf{L}$.

Night-flowering catchfly

Uncommon. Open hillsides and roadsides, on sandy soils. (+)

TRT-Dys, Hin; S.R.-Sno.

Silene pratensis (Rafn) Godron and Gren.

White campion

Lychnis alba Miller

S. alba (Miller) E. H. Krause

Fairly common. Waste areas and disturbed sites. $(+)$

APM-Ngt; TRT-Dys, Hct. 
S. cucubalus Wibel

Common. Rock crevices and various sandy sites. (+)

TRT-Crd, Dys, Gfd, Mnd, Sno.

Spergula arvensis $\mathbf{L}$.

Corn spurrey

Rare. Two locations. On gravel at roadside, and in damp roadside

ditch. $(+)$

TRT - Crd, Sno.

Stellaria graminea $\mathrm{L}$.

Lesser stitchwort

Common. Dry or damp, grassy or sandy roadsides, and open hillsides. $(+)$ APM-Ngt; TRT -Crd, Dys, Gfd, Hct, Stn.

Stellaria longifolia Muhlenb. ex Willd.

Starwort

Rare. One location. Damp sandy roadside in large disturbed area; among many species of plants.

TRT-Hct.

Stellaria media (L.) Villars

Common chickweed

Rare. One location. Damp edge of woods at roadside. $(+)$

TRT-Mnd.

CERATOPHYLLACEAE

Ceratophyllum demersum $\mathrm{L}$.

HORNWORT FAMILY

Fairly common. Quiet waters of lakes and streams up to $1.5 \mathrm{~m}$ deep; floating below surface.

TRT-Dys, Lut, Mnd.

Ceratophyllum echinatum A. Gray

Coontail

Common. Pools, stream edges, and quiet bays; in shallow water up to $0.7 \mathrm{~m}$ deep; floating at and below the surface.

TRT-Crd, Dys, Hin, Mnd; S.R.-Glm.

NYMPHAEACEAE

Brasenia schreberi J. F. Gmelin

WATER-LILY FAMILY

Water-shield

Common. Still or slow-flowing waters up to $1 \mathrm{~m}$ deep; leaves floating on surface.

TRT-Crd, Dys, Shb; S.R.-Glm, Hin, McC.

Nuphar pumila (Timm) DC.

Yellow water-lily

$N$. lutea (L.) Sibth. subsp. pumila (Timm) E. O. Beal

N. microphylla (Pers.) Fern.

Rare. One location. Open water of lake at 1.0 to $1.5 \mathrm{~m}$ depth; leaves

floating on surface.

TRT $-\mathrm{McC}$. 
$N$. lutea (L.) Sibth. subsp. variegata (Engelm. ex Durand in Clinton)

E. O. Beal

Common. Quiet or gently flowing waters of rivers, bays, and lakes; in shallow water up to $2 \mathrm{~m}$ deep; leaves floating on surface.

TRT-Dys, Hct, Mnd, Stn; S.R.-Crd, Hin, Liv.

Nymphaea odorata Dryander ex Aiton

White water-lily

Common. Small lakes, ponds, and quiet waters of rivers; in water up to $2 \mathrm{~m}$ deep; leaves floating on surface.

DFB-Lwr; TRT-Dud, Dys, Gfd, Hct, Hin, McC, Shb, Sno.

\section{RANUNCULACEAE}

Aconitum napellus $\mathbf{L}$.

CROWFOOT FAMILY

Monkshood

Rare. One location. Damp ground in partial shade of a stand of balsam-fir; escape from cultivation. $(+)$

TRT-Mnd.

Actaea pachypoda Elliott

A. alba (L.) Miller

White baneberry, Doll's-eyes

Common. Dry to damp deciduous woods; usually growing singly.

TRT-Crd, Gfd, Hav, Hbn, Hct, Mnd.

Actaea rubra (Aiton) Willd.

Red baneberry

Uncommon. Damp mixed or deciduous woods; singly or in small colonies.

TRT-Hbn, Mnd, Stn.

Anemone canadensis L.

Canada anemone

Rare. Two locations. Beside trail in woods, and in wide flat ditch.

TRT-Crd, Hct.

Anemone cylindrica A. Gray

Thimbleweed

Uncommon. Open grassy areas, often on low moist ground.

TRT-Dys.

Anemone riparia Fern.

Thimbleweed

Rare. Two locations. Open, dry grassy areas.

TRT-Hin, Mnd.

Anemone virginiana $\mathrm{L}$.

Thimbleweed

Fairly common. Open grassy slopes and clearings.

TRT-Mnd; S.R.-Lut. 
Common. Deciduous woods, clearings, and rock outcrops.

TRT-Crd, Hbn, Hct, Mnd; TRTE-Ans.

Aquilegia vulgaris $\mathrm{L}$.

Garden columbine

Rare. One location. Edge of deciduous woods; escape from cultivation,

well established. (+)

TRT-Mnd.

Caltha palustris $\mathrm{L}$.

Marsh-marigold

Rare. One location. In low, wet ditch on edge of mixed woods.

TRT-Mon.

Clematis virginiana $\mathrm{L}$.

Virgin's-bower

Fairly common. Climbing on shrubs, small trees, and low vegetation.

TRT-Dud, Hbn, Hct, Mon.

Coptis trifolia (L.) Salisb.

Goldthread

Common. Damp mixed woods and hemlock forests, on humus.

TRT-Gfd, Hbn, Hct, Liv, Mnd, Shb, Stn.

Hepatica acutiloba DC.

Sharp-leaved hepatica

Uncommon. Mixed or deciduous woods, on humus.

TRT-Dys; S.R.-Mnd.

Hepatica americana (DC.) Ker Gawler

Round-lobed hepatica

Uncommon. Deciduous woods, on rich humus.

APM-Ngt; TRT-Dys; S.R.-Mnd.

Ranunculus abortivus $\mathrm{L}$.

Kidneyleaf buttercup

Common. Wet or dry edges of woods, swampy areas, and along trails.

TRT-Crd, Dys, Hct, Stn.

Ranunculus acris $\mathrm{L}$.

Tall buttercup

Common. Roadsides, and occasionally in woods. (+)

APM-Ngt; TRT-Crd, Hav, Hbn, Hct, Shb, Sno; S.R.-Lut.

Ranunculus gmelini DC.

Yellow water-crowfoot var. hookeri (D. Don) Benson

Rare. Two locations. Mostly submersed in large shallow pool, and on wet muddy bank of small pool.

TRT -2 Crd.

Rare. One location. Shallow water at lake outlet.

TRT-Dud. 
Common. Wet muddy banks, ditches, and stream edges.

TRT-Crd, Hct, Mnd, Mon, Stn.

Ranunculus recurvatus Poiret ex Lam.

Fairly common. Damp or dry mixed woods.

APM-Brt; TRT-Crd, Hbn, Hct, McC; S.R.-Glm, Sno.

Ranunculus repens $\mathbf{L}$.

Creeping buttercup

Uncommon. Damp, partly shaded roadsides; in dense colonies. (+)

TRT-Stn.

Ranunculus reptans $\mathrm{L}$.

Creeping spearwort

var. reptans

Fairly common. Sandy shores and flats at water-line; in dense matted patches.

TRT-Hbn, Hct, Shb, Stn; S.R.-Hav.

var. ovalis (Bigelow) Torrey and A. Gray

Rare. Two locations. Low stream banks.

TRT-Mnd, Stn.

Ranunculus sceleratus $\mathrm{L}$.

Cursed buttercup

Uncommon. Waste areas, gravel roadsides, and rock depressions.

TRT-Crd, Mnd; S.R.-Hbn.

Thalictrum dasycarpum Fisch. and Avé-Lall.

Uncommon. Mucky shores and stream banks.

Purple meadow-rue

TRT-Hbn, Hct, Stn.

\section{Thalictrum dioicum $\mathbf{L}$.}

Rare. One location. Steep rocky hillside.

TRT-Mnd.

\section{Thalictrum pubescens Pursh}

T. polygamum Muhlenb.

Tall meadow-rue

Common. Wet meadows and damp stream banks.

TRT-Brt, Hct, Mnd; S.R.-Hin.

BERBERIDACEAE

Caulophyllum thalictroides (L.) Michaux

Early meadow-rue

Fairly common. Deciduous or mixed woods, on humus.

APM-Brt; TRT-Hbn, Hct, Mon; S.R.-Dys, Lut. 
Rare. One location. Under trees beside open waste ground. (+)

TRT-Dys.

Sanguinaria canadensis L.

Bloodroot

Rare. One location. Low, moist roadside on edge of deciduous woods; colony of around two hundred plants.

TRT-Mnd.

FUMARIACEAE

FUMITORY FAMILY

Corydalis sempervirens (L.) Pers.

Pale corydalis

Common. Dry sandy sites, especially on rocky shores and outcrops.

TRT-Crd, Dys, Hbn, Hct, Shb.

Dicentra canadensis (Goldie) Walp.

Squirrel-corn

Fairly common. Moist mixed woods, on leafy humus.

APM-Brt; TRT-Crd, Hct, Mnd.

Dicentra cucullaria (L.) Bernh.

Dutchman's breeches

Common. Moist mixed woods, on leafy humus.

TRT-Gfd, Hbn, Hct; S.R.-Dys, Mnd.

BRASSICACEAE

MUSTARD FAMILY

(CRUCIFERAE)

Alyssum alyssoides (L.) L.

Alyssum

Uncommon. Sandy and gravelly banks, and occasionally roadsides. $(+)$

TRT-Sno.

Arabis divaricarpa Nels.

Rock cress

Uncommon. Soil pockets on rocks, and sand-and-gravel flats.

TRT-Lut, Stn.

Arabis glabra (L.) Bernh.

Tower mustard

Rare. One location. Soil pockets on rock ledges.

TRT-Mnd.

Arabis hirsuta (L.) Scop.

Rock cress

var. pycnocarpa (M. Hopk.) Rollins

Rare. One location. Damp shady lakeshore.

TRT - Hct.

Barbarea vulgaris $\mathbf{R}$. Br.

Winter cress

Common. Moist roadsides and fields, among grasses. (+)

TRT-Gfd, Hct, Lut, Stn; S.R.-Sno. 
Rare. Two locations. Gravel bank, and abandoned railroad tracks on sand and cinders. $(+)$

TRT - Dys, Hct.

Brassica kaber (DC.) Wheeler

Charlock

Rare. One location. Dry sand fill over garbage dump. (+)

TRT-Dys.

Capsella bursa-pastoris (L.) Medikus

Common. Dry sandy sites and moist wooded roadsides. (+)

TRT-Dys, Hct, Mnd.

Cardamine pensylvanica Muhlenb. ex Willd.

Bitter cress

Common. Shaded pools and wet hollows in woods.

APM-Brt; TRT -Crd, Gfd, Hct, Mnd, Stn.

Dentaria diphylla Michaux

Shepherd's purse

Uncommon. Damp to wet mixed woods; colonies usually small.

APM-Lwr; TRT-Crd, Hbn.

Descurainia pinnata (Walter) Britton

Tansy-mustard

var. brachycarpa (Richardson) Fern.

Rare. One location. Beside abandoned railroad tracks.

TRT-Sno.

Descurainia sophia (L.) Webb ex Prantl

Herb-sophia

Rare. One location. Abandoned railroad tracks, on sand and cinders. $(+)$

TRT-Dys.

Diplotaxis tenuifolia (L.) DC.

Wall-rocket

Rare. One location. Beside abandoned railroad tracks, on sand and gravel. (+)

TRT-Dys.

Erysimum cheiranthoides $\mathrm{L}$.

Wormseed mustard

Fairly common. Wet, disturbed sandy sites. (+)

TRT-Crd, Dys, Hct, Mnd, Stn.

Hesperis matronalis $\mathrm{L}$.

Dame's rocket

Rare. One location. Partially shaded roadside; escape from

cultivation. (+)

TRT-Mnd. 
Lepidium campestre (L.) R. Br.

Field cress

Common. Open, grassy or rocky waste areas. $(+)$

TRT_Crd, Hct, Mnd; S.R._-Lut.

Lepidium densiflorum Schrader

Peppergrass

Fairly common. Dry, sandy waste ground. (+)

TRT-Dys, Sno, Stn.

Nasturtium microphyllum (Boenn.) Reichenb.

Water cress

Rare. One location. Small stream through wet lowland. (+)

TRT-Dys.

Rorippa palustris (L.) Besser

var. fernaldiana (Butters and Abbe) Stuckey

Uncommon. Damp sandy soils beside streams and roadsides.

TRT-Cly, Hbn; S.R.-Sno.

var. hispida (Desv.) Rydb.

Fairly common. Damp or wet meadows, and ditches and shorelines.

TRT-Crd, Dys, Hct, Shb, Stn.

Sisymbrium altissimum $\mathbf{L}$.

Tumble mustard

Rare. One location. Disturbed waste ground, on sand and gravel. (+)

TRT-Dys.

Sisymbrium officinale (L.) Scop.

Hedge mustard

Rare. One location. Sand fill over garbage dump. (+)

TRT-Dys.

Subularia aquatica L.

Awlwort

Rare. One location. Edge of low sand island in river.

DAO-Hav or Shb; MICH-Hav or Shb.

Thlaspi arvense $\mathrm{L}$.

Field penny-cress

Uncommon. Roadsides and old farmland, with grasses. (+)

TRT-Mnd; S.R.-Lut.

RESEDACEAE

MIGNONETTE FAMILY

Reseda lutea L.

Mignonette

Rare. One location. Sand and gravel roadside. (+)

TRT-Mnd. 
Fairly common. Sphagnum bogs and boggy edges of lakes.

TRT-Crd, Dud, Dys; S.R.-Hin, Liv, Stn.

\section{DROSERACEAE}

Drosera intermedia Hayne
SUNDEW FAMILY Spatulate-leaved sundew

Common. Partially submerged habitats on shorelines, flats, and old logs. APM-Lwr; TRT-Dys, Gfd, Hin, Shb, Sno, Stn.

Drosera rotundifolia $\mathbf{L}$.

Round-leaved sundew

Common. Bogs, shores, and damp sand flats; with mosses and grasses. TRT-Crd, Dud, Hbn, Hct, Hin, Shb.

CRASSULACEAE

ORPINE FAMILY

Sedum acre L.

Mossy stonecrop

Uncommon. Rock ledges and outcrops. (+)

TRT-Dys, Hin.

Sedum hispanicum L.

Spanish stonecrop

Rare. One location. Thin soil over rocks; escape from cultivation. (+) APM - Ngt.

Sedum telephium $\mathrm{L}$.

Live-forever

Uncommon. Dry waste sites and disturbed roadsides. (+)

DAO-Stn; TRT-Hct, Mnd, Stn.

\section{SAXIFRAGACEAE}

Chrysosplenium americanum Schwein.
SAXIFRAGE FAMILY

Golden water-carpet

Fairly common. Wet woods and shrub thickets; on muddy soil or loose sand.

APM-Brt; TRT -Crd, Gfd, Hct, Mnd, Stn.

Mitella diphylla $\mathbf{L}$.

Mitrewort

Common. Deciduous or mixed woods, on leafy ground, partially or completely shaded.

TRT-Crd, Hct, Mnd; S.R.-Dys, Lut.

Mitella nuda L.

Naked mitrewort

Common. Damp or wet areas: swamps and edges of woods.

TRT-Crd, Mnd, Mon.

Philadelphus coronarius $\mathbf{L}$.

Mock-orange

Rare. One location. Woodland thicket; escape from cultivation. (+)

APM-Ngt. 
Common. Moist woodlands, often on sandy soil.

Prickly gooseberry

APM-Ngt; TRT_Gfd, Hav, Hct, Mnd, Shb.

Ribes glandulosum Grauer

Skunk currant

Common. Wet woods, damp slopes, and rocky banks.

TRT-Crd, Hct, Hin, Liv, Mnd.

Ribes hirtellum Michaux

Uncommon. Low, moist sites with herbs and shrubs.

Wild gooseberry

TRT -Crd, Dys.

Ribes lacustre (Pers.) Poiret

Bristly black currant

Fairly common. Edges of swamps, wet woods, and damp slopes.

TRT-Crd, Hct, Mnd.

Ribes rubrum $\mathbf{L}$.

Red currant

$R$. sativum (Reichenb.) Syme

Rare. One location. Steep disturbed river bank; escape from cultivation. $(+)$

TRT-Mnd.

Ribes triste Pall.

Swamp red currant

Fairly common. Damp woods and open swampy areas.

TRT_Hbn, Hct, Mnd; S.R.-Dys, Hin.

Saxifraga virginiensis Michaux

Early saxifrage

Rare. Two locations. Moss-covered pockets on rocky outcrops.

TRT-Gfd, Lut.

Tiarella cordifolia $\mathbf{L}$.

Foamflower

Common. Deciduous or mixed woods, on leafy humus.

TRT-Gfd, Hct, Mnd; S.R.-Crd, Glm, Hin, Liv.

\section{ROSACEAE}

ROSE FAMILY

Agrimonia gryposepala Wallr.

Agrimony

Uncommon. Moist, open or sparsely treed areas.

TRT-Dys, Stn; S.R.-Mnd.

Amelanchier arborea (Michaux f.) Fern.

Serviceberry

Rare. Two locations. Shrubby edge of parking lot, and lakeshore.

TRT-Glm, Lut.

Amelanchier bartramania (Tausch) Roemer

Serviceberry

Rare. One location. Dry grassy hillside.

TRT-Mnd. 
Common. Roadsides, edges of woods, and open, often rocky sites.

TRT-Crd, Glm, Hbn, Hct, Mnd; S.R.-Dys.

Amelanchier sanguinea (Pursh) DC.

var. sanguinea

Common. Rocky areas, road banks, lake edges, and open areas in woods.

TRT-Hct, Mnd, Shb; S.R.-Dys, Hin.

\section{var. grandiflora (Wieg.) Rehder}

Uncommon. Rocky shore, roadside, and woodland.

APM-Brt; TRT-Dys, Mnd.

Amelanchier spicata (Lam.) K. Koch

var. spicata

Serviceberry

Uncommon. Three locations. High rock ridge, edge of woods, and forest clearing.

TRT-Glm, Hin, Mnd.

var. stolonifera (Wieg.) Cinq-mars

Rare. One location. Smooth, sloping rock shoreline.

TRT-Hin.

Aronia prunifolia (Marshall) Rehder

A. melanocarpa (Michaux) Elliott (see Voss, 1985)

Chokeberry

Common. Shores of lakes and ponds, edges of wet woods, and shrubby

hillsides.

TRT-Ans, Gfd, Hin, Liv, Mnd, Shb, Sno.

Crataegus macracantha Lodd.

Hawthorn

var. macracantha

Uncommon. Shrub thickets beside roads, and edges of deciduous woods.

TRT-Dys, Mnd.

var. occidentalis (Britton) Egglest.

Rare. One location. Edge of open field adjacent to deciduous woods.

TRT-Mnd.

Crataegus macrosperma Ashe

Hawthorn

Fairly common. Fencerows and open meadows.

TRT-Dys, Mnd, Stn.

Crataegus punctata Jacq.

Hawthorn

Uncommon. Open fields and woods bordering farmlands.

TRT-Dys, Mnd. 
Rare. One location. Sparsely wooded bank on sideroad.

TRT-Sno.

\section{Dalibarda repens $\mathbf{L}$.}

Dewdrops

Fairly common. Damp deciduous woods and wet thickets.

DFB-Ngt; TRT - Hct, Hin, Mnd, Shb, Stn.

Filipendula rubra (Hill) Robinson

Queen-of-the-prairie

Rare. Two locations. Damp bank of ditch, and sedge meadow; escapes

from cultivation. $(+)$

TRT - Gfd, Stn.

Fragaria vesca $\mathrm{L}$.

var. americana (Porter) Staudt

Uncommon. Small patches in clearings.

TRT-Lut, Stn.

Fragaria virginiana Miller

Wood strawberry

Common. Roadsides, old fields, and open hillsides; chiefly on sandy soils.

APM-Ngt; TRT-Crd, Gfd, Hbn, Hct, Stn; S.R.-Glm, Sno.

Geum aleppicum Jacq.

Yellow avens

var. strictum (Aiton) Fern.

Fairly common. Deciduous woods and damp open areas; on rich soils. (+) TRT-Dys, Hct, Sno, Stn.

Geum canadense Jacq.

White avens

Uncommon. Moist woods and clearings.

TRT-Crd, Hbn, Mnd.

Geum laciniatum Murray

Avens

Rare. Two locations. Among shrubs beside swamp, and on sandy

lakeshore.

TRT-Sno, Stn.

Geum macrophyllum Willd.

Large-leaved avens

Rare. Two locations. Low gravelly lakeshore and damp roadside.

TRT - Hct, Mnd.

Geum rivale $\mathrm{L}$.

Purple avens

Uncommon. Wet meadows.

APM-Brt; TRT-Dys, Stn. 
Pyrus malus L.

Uncommon. Mixed woods and disturbed soil locations. (+)

APM-Lwr; TRT-Crd, Gfd.

Physocarpus opulifolius (L.) Maxim.

Ninebark

Rare. One location. Open grassy bank, with other shrubs.

TRT-Dys.

Potentilla anserina $\mathrm{L}$.

Silverweed

Rare. One location. Edge of parking lot, on dry sand.

TRT-Mnd.

Potentilla argentea $\mathrm{L}$.

Silvery cinquefoil

Common. Open, dry sandy areas: roadsides, waste places, and shores. $(+)$

TRT-Cly, Crd, Gfd, Hbn, Hin, Mnd, Stn.

Potentilla inclinata Villars

Cinquefoil

Uncommon. Moist roadside, and edges of disturbed open sites. (+)

TRT-Crd, Sno.

Potentilla norvegica $\mathrm{L}$.

Rough cinquefoil

Common. Roadsides, clearings in woods, and wet shores.

TRT - Hbn, Hct, Sno, Stn; S.R.-Cly.

Potentilla palustris (L.) Scop.

Marsh cinquefoil

Fairly common. Swampy and marshy shorelines, and wet meadows.

TRT-Dud, Gfd, Hct, Hin, Mnd; S.R.-Mon.

Potentilla recta $\mathbf{L}$.

Rough-fruited cinquefoil

Common. Open areas on banks and roadsides, and clearings in woods. $(+)$ TRT-Dys, Gfd, Hct, Mnd, Stn; S.R.-Crd, Sno.

Potentilla simplex Michaux

Common cinquefoil

Uncommon. Low, moist grassy sites; in creeping colonies.

TRT - Hin, Mnd, Stn.

Prunus nigra Aiton

Canada plum

Uncommon. Edges of woods, and fencerows.

APM-Ngt; TRT - Mnd.

Prunus pensylvanica L.f.

Pin cherry

Common. Roadsides, woodland slopes, and lake and stream banks; in pure stands or mixed with other trees and shrubs.

TRT-Crd, Gfd, Hbn, Hct, Mnd; S.R.-Hin. 
Prunus serotina Ehrh.

Black cherry

Uncommon. Roadsides, old fields, sand flats, and occasionally mature woods (a species disappearing because of earlier cutting and current diseases).

APM-Ngt; TRT-Mnd, Sno; S.R.-Mnd.

Prunus virginiana $\mathrm{L}$.

Choke cherry

Common. Roadsides among shrubs or deciduous trees, stream edges, and old fields.

APM-Ngt; TRT - Hbn, Hct, Mnd, Shb.

Rosa acicularis Lindley

Uncommon. Open and sparsely treed areas.

Prickly wild rose

APM-Ngt; TRT-Glm.

\section{Rosa blanda Aiton}

Smooth wild rose

Uncommon. On sand and clay or sand and gravel, in partly shaded locations.

TRT-Dys, Mnd.

Rosa eglanteria L.

Sweet-brier

R. rubiginosa $\mathrm{L}$.

Rare. One location. Open meadow, with other shrubs. (+)

TRT-Stn.

Rosa multiflora Thunb. ex Murray

Many-flowered rose

Rare. One location. Edge of woods, with other shrubs, on sand and clay soil; escape from cultivation. (+)

TRT-Mnd.

Rosa palustris Marshall

Swamp rose

Uncommon. Wet shores in swampy or marshy sites; singly or in small colonies.

TRT-Mnd; S.R.-Dud, Dys, McC.

\section{Rubus allegheniensis Porter}

Common blackberry

Common. Open dry hillsides, sand flats, damp woods, and roadsides.

TRT-Dud, Hct, Hin, Mnd, Shb; S.R.-Crd, Sno.

Rubus canadensis L.

Smooth blackberry

Rare. Two locations. White-flowered plant in campsite, and pink-flowered plant on edge of low, damp woods.

TRT-Crd, Gfd. 
Uncommon. Low creeping shrub on dry, open sandy or rocky sites.

TRT-Crd, Mnd, Stn.

\section{Rubus hispidus L.}

Rare. One location. Gravel bank above wet valley.

Swamp dewberry

TRT-Stn.

Rubus odoratus L.

Purple-flowering raspberry

Common. Shrubby roadside banks; in sandy, gravelly, or rocky ground.

TRT-Crd, McC, Mnd; S.R.-Brt, Lut.

Rubus pubescens Raf.

Dwarf raspberry

Common. Damp or wet woods, and open disturbed ground; on sand, clay, humus, or muck.

TRT-Gfd, Hct, Liv, Mnd, Mon.

Rubus setosus Bigelow

Bristly blackberry

Uncommon. Damp to dry sites, among grasses or low shrubs.

TRT-Crd, Hct, Shb.

Rubus strigosus Michaux

$R$. idaeus L. var. strigosus (Michaux) Maxim.

Common. Shrubby, sandy open areas, roadsides, and fields.

TRT-Hct, Mnd; S.R.-Cly, Crd, Dys, Sno.

Sorbaria sorbifolia (L.) A. Braun

Wild red raspberry

Rare. Two locations. Fencerow on sand, and partly shaded bank; escape from cultivation. $(+)$

TRT-Dys, Stn.

Sorbus americana Marshall

Mountain-ash

Fairly common. Wet or moist lakeshores, and roadsides with shrubs and young trees.

APM-Ngt; TRT-Dud, Hbn, Hct, Liv, McC, Mnd.

Sorbus decora (Sarg.) C. Schneider

Showy mountain-ash

Rare. Two locations. Rocky slope and damp lowland.

DFB-Ngt; TRT - Hct.

Spiraea alba Du Roi

Narrow-leaved meadowsweet

Uncommon. Open edges of wetlands and lakes, and moist fields.

TRT-Crd, Dys, Hct, Shb; S.R._Ans, Mon, Sno. 
Spiraea latifolia (Aiton) Borkh.

Broad-leaved meadowsweet

S. alba Du Roi var. latifolia (Aiton) Dippel

Common. Edges of marshes, lakes, and ponds, and open, sandy disturbed ground.

TRT-Crd, Gfd, Hbn, Hct, Hin, Stn.

Spiraea tomentosa $\mathbf{L}$.

Steeple-bush

Common. Sandy shorelines, damp sandy ditches, roadsides, and open waste areas; usually with other low shrubs.

APM-Ngt; TRT-Gfd, Hbn, Hct, Hin.

Waldsteinia fragarioides (Michaux) Tratt.

Barren strawberry

Rare. Two locations. Old bush road, and low clay bank in leafy litter.

TRT-Hbn, Liv.

\section{FABACEAE}

\section{Coronilla varia $\mathrm{L}$.}

Rare. One location. Disturbed roadside. (+)

TRT-Dys.

Lathyrus latifolius $\mathrm{L}$.

Rare. One location. Edge of woods beside road. (+)

TRT-Crd.

\section{Lathyrus palustris $\mathbf{L}$.} var. linearifolius Ser.

Rare. One location. Beside low swamp, on sand.

TRT-Hbn.

\section{Lathyrus pratensis $\mathrm{L}$.}

Rare. One location. Roadside among grasses. (+)

TRT-Dys.

Lathyrus sylvestris $\mathrm{L}$.

BEAN FAMILY

Crown-vetch

Everlasting pea

Vetchling

Rare One location. Dry meadow with shrubs; escape from cultivation. $(+)$ TRT-Hct.

Lotus corniculatus $\mathbf{L}$.

Bird's-foot trefoil

Uncommon. Clearings and roadsides; in dry, grassy or sandy areas. $(+)$

TRT-Crd, Hct, Mnd.

Medicago lupulina $\mathbf{L}$.

Black medick

Uncommon. Roadsides and disturbed areas. (+)

TRT-2 Dys, Gfd. 
Uncommon. Open grassy areas and sandy roadsides. $(+)$

TRT-Gfd, Stn.

Melilotus alba Medikus

White sweet clover

Common. Sandy roadsides and old fields; often in large dense colonies. $(+)$ TRT_Mnd, Stn; S.R._Crd, Dys, Glm, Sno.

Melilotus officinalis (L.) Pallas

Yellow sweet clover

Uncommon. Sandy roadsides. $(+)$

TRT-Mnd, Stn.

Robinia pseudo-acacia L.

Black locust

Rare. Two locations. Fencerow and river bank; on sandy soils. (+)

TRT-Mnd, Stn.

Trifolium aureum Pollich

Hop clover

T. agrarium $\mathrm{L}$.

Common. Roadsides, dry open fields, and occasionally stream banks. $(+)$

TRT-Crd, Dys, Gfd, Hct, Mnd, Stn.

Trifolium hybridum L.

Alsike clover

Uncommon. Dry sites: roadsides, fields, and abandoned railroad tracks. $(+)$

TRT-Dys, Mnd, Stn.

\section{Trifolium pratense $\mathrm{L}$.}

Red clover

Common. Clearings and old fields. (+)

TRT-Dys, Hct, Hin, Mnd, Shb, Stn; S.R._Ans, Sno.

\section{Trifolium repens $\mathbf{L}$.}

White clover

Common. Sandy roadsides, dry fields, and woodlands. (+)

TRT-Dys, Hct, Mnd, Stn; S.R.-Glm.

\section{Vicia angustifolia $\mathbf{L}$.}

V. sativa L. subsp. nigra (L.) Ehrh.

Rare. Two locations. Damp ditch with grasses and sedges, and abandoned railroad tracks on gravelly soil. $(+)$

TRT-Sno, Stn.

Vicia cracca $\mathbf{L}$.

Cow vetch

Common. Roadsides, and beside abandoned railroad tracks on sand and gravel. (+)

TRT-Dys, Gfd, Hct, Shb, Stn; S.R.-Sno. 
Vicia tetrasperma (L.) Schreber

Four-sided vetch

Uncommon. Dry open sites: rock ridges and waste ground. $(+)$

APM-Ngt; TRT-Lut, Sno.

OXALIDACEAE

WOOD-SORREL FAMILY

Oxalis montana Raf.

Wood-sorrel

Fairly common. Damp to swampy mixed woods.

TRT-Gfd, Hct, Mnd; S.R.-Crd.

Oxalis stricta $\mathbf{L}$.

Yellow wood-sorrel

Fairly common. Old lawns, driveways, and sandy roadsides.

TRT-Gfd, Mnd, Shb; S.R.-McC, Mnd.

GERANIACEAE

Geranium bicknellii Britton

Uncommon. Clearings and sandy disturbed sites.

TRT-Lut, Shb, Stn.
GERANIUM FAMILY

Bicknell's cranesbill

Geranium robertianum $\mathbf{L}$.

Herb-robert

Uncommon. Sandy, partially shaded roadsides and grassy, thinly wooded slopes.

TRT-Crd, Mnd, Mon, Stn.

POLYGALACEAE

Polygala paucifolia Willd.

Uncommon. Edge of damp woods, and wooded shores.

TRT-Ans, Shb; S.R.-Lut.

\section{Polygala polygama Walter}

Milkwort

Rare. One location. Sandy ground by small lake.

CAN-Glm.

\section{EUPHORBIACEAE}

Euphorbia cyparissias $\mathbf{L}$.

Rare. One location. Roadside in dense grass. (+)

TRT-Mnd.

Euphorbia glyptosperma Engelm.

MILKWORT FAMILY

Fringed polygala

Spurge

Uncommon. Sand, gravel, and cinder areas, and rock ledges.

TRT_Dys, Lut, Mnd, Shb, Sno.

Euphorbia helioscopia L.

Sun spurge

Rare. One location. Grassy open roadside. (+)

TRT-Dys. 
Rare. One location. Beside abandoned railroad track, in sand.

TRT-Dys.

CALLITRICHACEAE

Callitriche verna $\mathrm{L}$.

Uncommon. Pools and quiet streams.

TRT-Hct, Mnd; S.R.-Stn.

\section{ANACARDIACEAE}

Rhus radicans $\mathrm{L}$.

CASHEW FAMILY

Poison ivy

Uncommon. Dry gravel roadside on top of rocky hillside; very occasionally seen on edges of woods.

TRT-Mnd.

Rhus typhina L.

Staghorn sumac

Common. Rocky outcrops, grassy hillsides, and dry open roadsides.

TRT-Mnd, Shb; S.R.-Ans, Lut, Stn.

AQUIFOLIACEAE

Ilex verticillata (L.) A. Gray

HOLLY FAMILY

Winterberry

Fairly common. Banks of streams and lakes, and edges of marshes.

DAO-Shb; TRT-Hct, Mnd, Shb, Stn.

Nemopanthus mucronata (L.) Trel.

Mountain holly

Common. Low, wet areas: often shores of lakes, ponds, and streams, and wet thickets.

TRT-Dud, Gfd, Hct, Hin, Mnd; S.R.-Liv.

CELASTRACEAE

STAFF-TREE FAMILY

Celastrus scandens $\mathrm{L}$.

Bittersweet

Rare. One location. Climbing on wire fence on open bank.

TRT-Mnd.

ACERACEAE

Acer negundo $\mathrm{L}$.

MAPLE FAMILY

Fairly common. Parks, lawns, and waste places. $(+)$

TRT-Dys.

Acer pensylvanicum $\mathrm{L}$.

Striped maple

Fairly common. Moist deciduous woods, shaded roadsides, and lake edges.

TRT-Crd, Gfd, Hbn, Hct, Hin, Shb. 
Common. Wet or damp deciduous or mixed woods, and swamps.

TRT-Dud, Dys, Gfd, Hbn, Hct, Mnd, Shb; S.R.-Ans, Hin, Stn.

\section{Acer saccharinum $\mathbf{L}$.}

Silver maple

Uncommon. Low banks of lakes or streams; leaning over the water.

TRT-Mnd; S.R.-Sno.

\section{Acer saccharum Marshall}

Sugar maple

Common. In pure stands or in mixed forests; usually on leaf-covered, humus-rich soil; abundant throughout the county.

APM-Ngt; TRT - Hct, Mnd.

\section{f. rugelii (Pax.) Palmer and Steyerm.}

Rare. Two locations. Edges of damp woods at roadsides.

TRT -2 Mnd.

\section{Acer spicatum Lam.}

Fairly common. Edges of woods, banks, and partially open woodlands; on dry or moist soils.

TRT-Gfd, Hct, Mnd, Stn; S.R.-Hin.

\section{BALSAMINACEAE}

\section{Impatiens capensis Meerb.}

I. biflora Walter

Common. Damp or wet places, on muck or humus; usually in large colonies.

TRT-Crd, Gfd, Hct, Mnd, Shb, Stn; S.R.-Glm.

\section{RHAMNACEAE}

\section{Rhamnus alnifolia L'Her.}

BUCKTHORN FAMILY

Rare. One location. Low, damp ground between woods and bank of abandoned railroad track, with speckled alders.

TRT-Sno.

\section{VITACEAE}

Parthenocissus vitacea (Knerr) A. Hitchc.

GRAPE FAMILY

Common. Creeping on roadsides, abandoned railroad tracks, and open rocky areas; climbing on trees and poles; occasionally spreading into deciduous woods among dead leaves.

APM-Lwr, Ngt; TRT-Dud, Dys, Hct, Lut, Mnd. 
Common. Scattered in deciduous or mixed woods and on roadsides; occasionally growing singly in cleared areas.

TRT-Dys, Hct, Mnd, Shb; S.R.-Crd, Hin, Lut.

\section{MALVACEAE}

MALLOW FAMILY

Malva moschata L. Musk mallow

Uncommon. Open, dry sites with grasses and low herbs; in small colonies. $(+)$

APM-Ngt; TRT-Dys, Hct, Sno, Stn.

Malva neglecta Wallr.

Common mallow

Rare. One location. Vegetable garden. (+)

TRT-Mnd.

HYPERICACEAE

Hypericum boreale (Britton) Bickn.

ST. JOHN'S-WORT FAMILY

Northern St. John's-wort

Common. Wet, muddy or sandy edges of marshes and streams; in shallow water or on shore.

TRT -Dys, Gfd. Glm. Hbn. Hct. Hin, McC. Shb, Stn.

\section{f. callitrichoides Fassett}

Fairly common. A submersed form of $H$. boreale with differing leaf characters; sterile.

TRT-Hin, Shb; S.R.-Mnd.

Hypericum canadense $\mathrm{L}$.

Canadian St. John's-wort

Common. Edges of streams and other low, open, damp to wet areas; usually on muck.

TRT-Hct, Hin, Shb, Sno; S.R.-Dys, Glm.

Hypericum ellipticum Hook.

Pale St. John's-wort

Common. On damp banks of creeks and rivers, and in bogs on floating masses of herbaceous plants.

TRT-Crd, Gfd, Hct, Hin, Mnd; S.R.-Stn.

Hypericum majus (A. Gray) Britton

Large St. John's-wort

Rare. One location. Edge of lake, in shallow water about $10 \mathrm{~cm}$ deep.

TRT-Stn.

Hypericum mutilum $\mathrm{L}$.

Dwarf St. John's-wort

Rare. Two locations. Low, wet ground.

TRT -2 Shb. 
Common. Roadsides, fields, meadows, and open sandy areas: often in large colonies. (+)

TRT-Dys, Hct, Shb, Sno, Stn; S.R.-Ans, Cly, Crd, Glm, Sno.

\section{Triadenum fraseri (Spach) Gleason}

T. virginicum (L.) Raf. subsp. fraseri (Spach) J. M. Gillett

Marsh St. John's-wort Hypericum virginicum L. var. fraseri (Spach) Fern.

Common. Sandy edges of streams and lakes, and wet meadows, marshes. and bogs.

TRT-Dys, Gfd, Hbn. Hct, Hin, Stn; S.R.-Mon.

\section{ELATINACEAE}

Elatine minima (Nutt.) Fischer and C. Meyer

WATERWORT FAMILY Waterwort Rare. Three locations. Submersed in shallow water of river, and emersed on sandy shoreline. Rare in Ontario (Argus et al., 1982-1987). CAN-Hav or Shb; TRT-Stn; TRTE-Shb.

\section{CISTACEAE}

\section{Lechea intermedia Leggett}

ROCKROSE FAMILY

Rare. One location. Sandy pockets in granite outcrop.

TRT - Hct.

\section{VIOLACEAE}

\section{Viola adunca Smith}

VIOLET FAMILY

Rare. Two locations. Sandy banks in partial shade.

Hooked-spur violet

TRT -Crd, Sno.

\section{Viola blanda Willd.}

Sweet white violet

Uncommon. Deciduous woods, rock depression on roadside, and edge of meadow in shade of spruce trees.

TRT-Hbn, Mnd; S.R.-Mnd.

Viola canadensis $\mathrm{L}$.

Canada violet

Common. Deciduous or mixed woods and partially wooded clearings. APM-Brt; TRT -Dud, Dys, Gfd, Hbn, Hct, Mon, Stn.

Viola conspersa Reichenb.

American dog violet

Fairly common. Damp and wet areas, open or shaded; on various soils. TRT-Dys, Hbn, Hct, Mnd; S.R.-Crd.

\section{Viola cucullata Aiton}

Blue marsh violet

Common. Damp to wet ditches at edges of woods, and sand or mud shores: often in large dense colonies.

TRT-Dys, Gfd, Hbn, Hct, Mnd, Stn; S.R._Liv, Lut. 
Rare. One location. In a colony of mostly blue $V$. cucullata where colours ranged from white to deep blue.

TRT-Liv.

Viola incognita Brainerd

Large-leaved white violet

Fairly common. Damp to wet deciduous woods, wet depressions, and wooded banks.

TRT - Hbn, Hct, Hin, Mnd, Mon; S.R.-Dys.

Viola lanceolata $\mathrm{L}$.

Lance-leaved violet

Common. Open sandy or rocky shores, and edges of woods.

DFB-Ngt; TRT-Ans, Gfd, Glm, Hin, McC, Shb, Stn.

Viola nephrophylla E. Greene

Northern bog violet

Rare. Two locations. Mucky stream bed and rich woods.

TRT - 2 Hbn.

Viola pallens (Banks ex DC.) Brainerd

$V$. macloskeyi F. E. Lloyd var. pallens (DC.) C. L. Hitchc.

Common. Damp or wet woods, banks, lake edges, lawns, and meadows.

TRT - Hbn, Hct, Hin, Stn; S.R._-Dys, Hin.

\section{Viola pubescens Aiton}

var. pubescens

Downy yellow violet

Fairly common. Edges of deciduous woods and shaded roadsides; on humus or sand and gravel.

TRT_Dys, Hct, Mnd; S.R.-Dys.

var. leiocarpa (Fern. and Wieg.) Boiv.

Smooth yellow violet

$V$. eriocarpa Schwein.

Uncommon. Deciduous woods, on humus.

TRT-Dys, Gfd, Hbn.

Viola renifolia A. Gray

Kidney-leaved violet

Fairly common. Damp mixed woods.

TRT-Dys, Gfd, Hbn, Hct, Hin, Mnd; S.R.-Crd, Glm.

Viola selkirkii Pursh ex Goldie

Great-spurred violet

Fairly common. Damp to wet chiefly deciduous woods, and edges of streams.

TRT-Crd, Gfd, Hbn, Hct, Mnd; S.R.-Dys. 
Viola septentrionalis E. Greene

Northern blue violet

Common. Edges of woods, open woodlands, thickets, and sandy roadsides.

TRT-Dys, Gfd, Hbn, Hct, Mnd, Stn; S.R.-Hin.

f. alba Vict. and Rouss.

Rare. Two locations. Wet lumbering road and grassy park.

TRT-Dys, Hct.

Viola sororia Willd.

Woolly blue violet

Uncommon. Open or shaded, damp or dry sites.

TRT-Gfd, Hbn, Hct, Stn.

Viola tricolor L.

Johnny-jump-up

Rare. Two locations. Edge of damp woods and open ditch; escapes from

cultivation. $(+)$

TRT-Ans, Dys.

THYMELAEACEAE

MEZERUM FAMILY

Dirca palustris L.

Leatherwood

Uncommon. Damp woods, and occasionally sparsely wooded hillsides.

DAO-Hct; TRT-Mnd; UWO-Glm; S.R.-Lut.

ELAEAGNACEAE

OLEASTER FAMILY

Shepherdia canadensis (L.) Nutt.

Buffalo-berry

Rare. Two locations. Woods at edges of small clearings.

TRT-2 Lut.

\section{LYTHRACEAE}

Decodon verticillatus (L.) Elliott
LOOSETRIFE FAMILY

Swamp loosestrife var. laevigatus Torrey and A. Gray

Uncommon. In shallow water offshore or in floating mats in lakes; in dense colonies spreading by thick underwater stems.

TRT-Hin, Lut, Mnd.

Lythrum salicaria $\mathrm{L}$.

Purple loosestrife

Rare. Two locations. Low, wet sites among shrubs and grasses. $(+)$

TRT-Dys; S.R.-Shb.

\section{MELASTOMACEAE}

MELASTOMA FAMILY

Rhexia virginica $\mathrm{L}$.

Meadow-beauty

Rare. One location. Damp sandy shore of small lake. Rare in Ontario

(Argus et al., 1982-1987).

TRT-Hin. 
Fairly common. Wet or damp coniferous or mixed woods, and often edges of creeks or pools.

TRT-Gfd, Hbn, Hct, Mnd, Ngt.

Circaea quadrisulcata (Maxim.)

Enchanter's nightshade

Franchet and Savat.

C. lutetiana $\mathrm{L}$. var. canadensis $\mathrm{L}$.

Uncommon. Open coniferous woods and moist mixed woods.

TRT-Gfd, Lut, Stn.

Epilobium angustifolium $\mathbf{L}$.

Fireweed

Common. Roadsides, wastelands, and edges of open, dry or wet clearings. APM-Ngt; TRT-Crd, Dys, Gfd; S.R._Glm, Hin, Sno.

Epilobium ciliatum Raf.

Willow-herb

Common. Moist to damp sites in open areas, often roadside ditches.

TRT-Brt, Gfd, Hct, Lut, Mnd, Shb, Stn.

Epilobium coloratum Biehler

Willow-herb

Rare. Two locations. Swampy water's edge and dry disturbed roadside.

TRT - Hct, Mnd.

Epilobium glandulosum Lehm.

Willow-herb

E. ciliatum Raf. subsp. glandulosum (Lehm.) Hoch and Raven

Rare. One location. Clearing in mixed woods, on bank.

TRT-Hbn.

Epilobium hirsutum L.

Hairy willow-herb

Rare. One location. Low, wet waste area. (+)

TRT -Stn.

Epilobium leptophyllum Raf.

Narrow-leaved willow-herb

Uncommon. Damp ditches and open sedge and grass areas.

APM-Cly; TRT-Crd, Mnd, Sno.

Epilobium palustre L.

Willow-herb

Rare. One location. Damp, flat open peninsula in lake.

TRT-Shb.

Ludwigia palustris (L.) Elliott

Water-purslane

Uncommon. Creeping on wet shores, and floating in adjacent shallow waters.

TRT-Crd, Sno; S.R.-Hin, Lut. 
Common. Roadsides, open hillsides, sand flats, and clearings in woods.

TRT-Hct, Mnd, Shb; S.R.-Brt, Crd, Dys, Glm, Sno.

\section{Oenothera parviflora $\mathrm{L}$.}

Small-flowered evening primrose

Uncommon. On sand and gravel on disturbed ground.

TRT-Dys, Hct, Hin, Stn.

Oenothera perennis $\mathbf{L}$.

Sundrops

Uncommon. Old fields and sandy or grassy lake edges.

TRT-Crd, Hct, Mnd, Stn.

HALORAGIDACEAE

WATER-MILFOIL FAMILY

(HALORAGACEAE)

Myriophyllum alterniflorum DC.

Water-milfoil

Fairly common. Quiet shallow water; in massed colonies with fruiting spikes emersed.

TRT-Crd, Dys, Mnd; S.R.-Gfd, Lut.

Myriophyllum exalbescens Fern.

Water-milfoil

Rare. One location. Offshore in bay in water $80 \mathrm{~cm}$ deep; in massed colony with emersed fruiting spikes.

TRT-Gfd.

Myriophyllum farwellii Morong

Water-milfoil

Uncommon. Offshore in lakes, bays, ponds, and wide streams; entirely submersed with fruiting bodies in leaf axils.

TRT-Crd, Hin, Lut, Mnd.

Myriophyllum spicatum L.

Eurasian water-milfoil

Uncommon. Submersed plants in shallow water of lakes, with emersed

flowering spikes; introduced and spreading. (+)

TRT-Crd, Mnd.

Myriophyllum tenellum Bigelow

Water-milfoil

Uncommon. Near shoreline in sheltered bays of lakes and streams; emersed flowering stems.

APM-Lwr; DFB-McC; TRT-Liv, Stn.

Myriophyllum verticillatum $\mathrm{L}$.

Water-milfoil

Uncommon. Quiet shallow waters of lakes and bays; flowering spikes emersed.

TRT-Crd, Lut, Mnd. 
Rare. Two locations. In slow current of shallow river and in boggy pool; submersed plants with emersed flowering branches.

TRT-Dys, Mnd.

\section{HIPPURIDACEAE}

MARE'S-TAIL FAMILY

Hippuris vulgaris $\mathrm{L}$.

Mare's-tail

Uncommon. Offshore in quiet shallow water; entirely or partly submersed. TRT -Crd, Hct, Shb.

\section{ARALIACEAE}

Aralia hispida Vent.

GINSENG FAMILY

Bristly sarsaparilla

Fairly common. Edges of ponds, streams, and woods, and soil pockets on rocky lake banks; on damp sandy soils.

TRT-Cly, Hbn, Hin, Mnd, Shb.

Aralia nudicaulis L.

Wild sarsaparilla

Common. Woods, partly shaded hillsides, and sandy disturbed sites.

TRT-Gfd, Hct, Shb, Stn; S.R.-Crd, Hin.

\section{Aralia racemosa $\mathrm{L}$.}

Spikenard

Common. Shrubby borders of woods and thickets.

TRT-Crd, Gfd, Hbn, Hct, McC, Mnd, Shb.

\section{Panax quinquefolius L.}

Ginseng

Rare. Two locations. Maple forest on damp humus-rich soil, and edge of dense mixed woods.

TRT-Crd, Lut.

APIACEAE

PARSLEY FAMILY

(UMBELLIFERAE)

Aegopodium podagraria L.

Goutweed

Rare. One location. Open, damp roadside; escape from cultivation. (+)

TRT-Mnd.

Carum carvi L.

Caraway

Rare. Two locations. Sedge meadow and roadside with grasses. (+)

TRT-Mnd, Sno.

Cicuta bulbifera $\mathbf{L}$.

Water-hemlock

Fairly common. Wet sites: low open banks, stream edges, lakeshores, and swamps.

TRT -Crd, Dys, Gfd, Hct, Mnd; S.R.-Mon. 
Cryptotaenia canadensis (L.) DC.

Honewort

Rare. One location. Clearing in woods.

TRT-Gfd.

Daucus carota $\mathbf{L}$.

Queen Anne's-lace

Uncommon. Waste areas, and open sandy roadsides. $(+)$

TRT-Mnd, Stn.

Heracleum mantegazzianum Sommier and Levier

Rare. One location. Dry roadside. (+)

TRT-Hct.

Heracleum maximum Bartram

Cow parsnip

$H$. lanatum Michaux

Rare. Two locations. In mud-and-gravel ditch beside abandoned railroad, and in rank vegetation on hillside.

APM-Ngt; S.R. - Hct.

Hydrocotyle americana $\mathrm{L}$.

Water-pennywort

Fairly common. Wet depressions in open sandy sites, and wet pockets of moss on rocky slopes in woods.

TRT-Crd, Hbn, Shb, Stn.

Osmorhiza claytoni (Michaux) C. B. Clarke

Sweet cicely

Fairly common. Bush roads, trails, and thin deciduous woods; often in large colonies.

APM-Brt; TRT -Crd, Hct, Hin, Mnd.

Pastinaca sativa $\mathrm{L}$.

Wild parsnip

Rare. One location. Abandoned railroad tracks, in gravel and cinders. $(+)$

TRT-Sno.

Pimpinella saxifraga L.

Burnet-saxifrage

Rare. One location. Low grassy bank beside road. (+)

TRT -Stn.

Sanicula marilandica $\mathrm{L}$.

Black snakeroot

Uncommon. On roadsides, at edges of woods, and beside bush paths; in partial shade.

TRT-Crd, Dys; S.R.-Mnd.

Sium suave Walter

Water parsnip

Uncommon. Low, wet shaded areas; sometimes in standing water, on mud or muck.

TRT-Crd, Dys, Mnd; TRTE-Stn. 
Common. Shores of lakes and edges of deciduous or mixed woods.

TRT-Crd, Gfd, Hbn, Hct, Shb; S.R.-Hin, Lut.

\section{Cornus canadensis $\mathbf{L}$.}

Bunchberry

Common. Deciduous and mixed woods and shaded banks.

TRT_Gfd, Hbn, Hct, Liv, Shb.

Cornus obliqua Raf.

C. amomum Miller subsp. obliqua (Raf.) J. S. Wilson

Silky dogwood

Rare. Two locations. Wet edge of small lake, and damp ditch; colonies large and dense.

TRT-Dys, Gfd.

Cornus rugosa Lam.

Round-leaved dogwood

Uncommon. At edges of woods, on high banks, or near water-lines.

TRT-Crd, Lut, Stn.

\section{Cornus stolonifera Michaux}

Red osier dogwood

Common. Stream banks, lake edges, and damp ditches.

TRT-Hct, Mnd, Shb; S.R.-Crd, Dys, Lut, Mon.

\section{PYROLACEAE}

Chimaphila umbellata (L.) Barton

PYROLA FAMILY

Uncommon. Sparsely wooded, usually rocky areas.

Pipsissewa

TRT_Hbn, Hct, Mnd, Shb; S.R.-Dys.

Moneses uniflora (L.) A. Gray

One-flowered wintergreen

Rare. Two locations. Mossy hummock in dense, wet cedar-balsam swamp, and cedar-black ash swamp.

TRT-Mnd; UWO-Glm.

Monotropa hypopithys L.

Pinesap

Uncommon. Damp deciduous woods, on moist humus.

TRT-Dys, Hin, Lut, Shb.

Monotropa uniflora $\mathbf{L}$.

Indian-pipe

Fairly common. Cool mixed woods, on moist humus.

TRT-Hct, Mnd, Shb; S.R.-Dys, Hin, Liv.

Pyrola asarifolia Michaux

Pyrola

\section{subsp. asarifolia}

Rare. Two locations. Damp to wet mixed woods.

TRT-Crd, Mnd. 


\section{subsp. americana (Sweet) Krisa}

$P$. americana Sweet

Rare. Two locations. Damp woods, on acid soil.

TRT-Crd, Shb.

Pyrola chlorantha Sw.

Green pyrola

$P$. virens Schweigg.

Uncommon. Moist mixed woods.

TRT-Crd, Hct, Lut.

Pyrola elliptica Nutt.

Shinleaf

Common. Tall forests, on humus; and drier, open coniferous or deciduous woods.

APM-Ngt; TRT -Crd, Gfd, Hct, Mnd, Mon, Shb.

\section{Pyrola secunda $\mathbf{L}$.}

Orthilia secunda (L.) House

One-sided pyrola

Fairly common. Damp coniferous or mixed woods, usually in deep shade.

DFB-Ngt; TRT-Dys, Hct, Shb.

\section{ERICACEAE}

\section{Andromeda glaucophylla Link}

A. polifolia L. subsp. glaucophylla (Link) Hultén

HEATH FAMILY

Bog-rosemary

Fairly common. Bogs and low, shrubby borders of lakes and streams.

TRT-Ans, Crd, Dys, Hct, McC.

Arctostaphylos uva-ursi (L.) Sprengel

Bearberry

Uncommon. Sandy pockets on rock outcrops.

TRT-Hct, Mnd.

Chamaedaphne calyculata (L.) Moench

Leatherleaf

Common. Bogs and wet lakeshores.

TRT-Dud, Dys, Hbn, Hct, Shb; S.R.-Crd, Hin, Mon.

\section{Epigaea repens $\mathbf{L}$.}

Trailing-arbutus

Fairly common. Edges of deciduous woods, moss-covered pockets on rock ridges, and rocky shaded banks under conifers.

TRT-Crd, Gfd, Glm, Hbn, Shb; S.R.-Liv, Mon.

Gaultheria hispidula (L.) Muhlenb. ex Bigelow Creeping snowberry

Chiogenes hispidula (L.) Torrey and A. Gray

Fairly common. Damp, mostly coniferous woods and wet, shaded shorelines.

TRT - Dud, Hct, Mnd, Shb. 
Common. Damp coniferous or mixed woods and shaded mossy banks.

TRT-Crd, Gfd, Hct, Shb, Stn; S.R.-Dys, Hin.

Gaylussacia baccata (Wangenh.) K. Koch

Rare. One location. Rocky shoreline, under tall shrubs.

TRT-Dud.

Kalmia angustifolia $\mathbf{L}$.

Sheep-laurel

Rare. Two locations. Edge of swamp and boggy area beside pond.

TRT-Dud, Hin.

Kalmia polifolia Wangenh.

Bog-laurel

Common. Wet, boggy edges of lakes, ponds, and streams; often with other low shrubs on sphagnum.

TRT-Ans, Crd, Dys, Gfd, Liv, McC, Shb; S.R._Hct, Hin.

\section{Ledum groenlandicum Oeder}

Labrador tea

Fairly common. Swamps, bogs, and wet openings in coniferous woods; usually on sphagnum.

TRT-Dud, Hbn, Hct, Hin, McC, Mnd; S.R.-Mon.

Vaccinium angustifolium Aiton

Low sweet blueberry

Fairly common. Rocky shores, rock knolls, open hillsides, and old bush roads.

TRT_Dys, Glm, Hbn, Hct; S.R._Hin, Liv.

\section{Vaccinium macrocarpon Aiton}

Large cranberry

Fairly common. Sphagnum mats in bogs, and boggy edges of small lakes.

TRT-Dys, Gfd, Hin, Shb.

\section{Vaccinium myrtilloides Michaux}

Common. Bogs and clearings in woods.

APM-Ngt; TRT-Crd, Dys, Gfd, Hbn, Hct, Mnd, Shb.

Vaccinium oxycoccus $\mathbf{L}$.

Velvet-leaf blueberry

Uncommon. Bogs and swamps; usually creeping over sphagnum mats.

TRT_Dys, Hct; S.R._Hin, Mnd.

PRIMULACEAE

Lysimachia ciliata $\mathbf{L}$.

Steironema ciliatum (L.) Raf.

Rare. One location. Among shrubs on edge of woods.

TRT-Mnd.
PRIMROSE FAMILY

Fringed loosestrife 
Rare. Two locations. Open sandy sites at edges of woods. (+)

APM-Ngt; TRT-Mnd.

Lysimachia terrestris (L.) Britton, Sterns, and Pogg.

Swamp-candles

Common. Wet shorelines of lakes and ponds; on muck or sand.

APM-Cly; TRT-Crd, Gfd, Hct, Mnd, Shb, Stn.

Lysimachia thyrsiflora $\mathrm{L}$.

Tufted loosestrife

Naumbergia thyrsiflora (L.) Duby

Fairly common. Marshes and bogs, and borders of streams, lakes, or ponds; on shore or in shallow water.

TRT-Gfd, Hct, Mnd; S.R.-Dys, Mon.

Trientalis borealis Raf.

Starflower

Common. Damp woods, wooded or open banks, and soil pockets on rocky ridges.

TRT -Dys, Gfd, Shb, Stn; S.R._-Glm, Hin, Liv.

OLEACEAE

Fraxinus americana $\mathrm{L}$.

OLIVE FAMILY

Fairly common. Deciduous or mixed woods.

White ash

APM-Brt; TRT-Dys, Hct, Mnd; TRTE-Ans.

Fraxinus nigra Marshall

Black ash

Fairly common. Wet lake edges and swamps.

TRT-Dud, Dys, Mnd; S.R.-Mon.

Fraxinus pennsylvanica Marshall

Red ash

Uncommon. Open deciduous woodlands.

APM-Brt; TRT-Hct, Shb, Sno.

Syringa vulgaris $\mathrm{L}$.

Common lilac

Uncommon. Partially open woodlands; introduced and established. (+)

APM-2 Ngt; S.R.-Sno.

GENTIANACEAE

Gentiana linearis Froelich

GENTIAN FAMILY

Uncommon. Damp grassy roadsides, partly shaded or open.

TRT-Hbn, McC, Sno.

Menyanthes trifoliata $\mathbf{L}$.

Buckbean

Uncommon. Sphagnum mats in bogs and boggy edges of small lakes and slow streams.

TRT-Crd, Dys; S.R.-McC. 
Uncommon. Quiet waters of ponds and bays, and quiet river backwaters; leaves floating on surface.

TRT_Gfd, Hin, Shb, Stn.

\section{APOCYNACEAE}

Apocynum androsaemifolium $\mathrm{L}$.

DOGBANE FAMILY Spreading dogbane

Common. Roadsides, rocky shores, and dry clearings.

TRT -Cly, Gfd, Hct, Hin, Mnd; S.R._Ans, Ngt, Sno.

\section{Apocynum cannabinum $\mathbf{L}$.}

Indian hemp

Rare. One location. Shrubby edge of gravel sideroad.

TRT-Mnd.

Vinca minor L.

Periwinkle

Rare. Two locations. Rocky roadside bank and large, flat grassy clearing; escapes from cultivation. (+)

TRT-Glm, Mnd.

ASCLEPIADACEAE Asclepias incarnata $\mathrm{L}$.

MILKWEED FAMILY Swamp milkweed

Uncommon. Swamps, marshes, and low, wet banks.

TRT -Hbn, Hct, Mnd, Stn.

Asclepias syriaca $\mathbf{L}$.

Common milkweed

Common. Dry or damp roadside ditches, open hillsides, and old fields.

TRT-Dys, Mnd, Stn; S.R._Lut.

CONVOLVULACEAE

MORNING-GLORY FAMILY

Convolvulus arvensis $\mathrm{L}$.

Field bindweed

Rare. Two locations. Open grassy bank and old farm field. (+)

TRT-Dys, Stn.

Convolvulus sepium $\mathrm{L}$.

Hedge bindweed

Rare. Two locations. Dry sand flats and low, wet area.

TRT-Dys, Stn.

POLEMONIACEAE

PHLOX FAMILY

Phlox paniculata $\mathrm{L}$.

Phlox

Rare. Two locations. Open grassy areas; escapes from cultivation, established. (+)

TRT-Mnd; S.R.-Hct. 
Phlox subulata L.

Moss-pink

Rare. One location. Low roadside bank; escape from cultivation. $(+)$

TRT-Mnd.

BORAGINACEAE

Cynoglossum boreale Fern.

BORAGE FAMILY

Wild comfrey

Rare. One location. Edge of woods above swamp.

TRT-Dys.

Cynoglossum officinale $\mathrm{L}$.

Uncommon. Dry roadsides and old dry fields. (+)

Hound's-tongue

TRT - Hct, Mnd, Stn.

Echium vulgare $\mathrm{L}$.

Common. Waste areas, roadsides, and old fields. (+)

TRT-Mnd, Stn; S.R.-Crd, Dys, Mon.

Viper's bugloss

Hackelia americana (A. Gray) Fern.

Stickseed

H. deflexa (Wahlenb.) Opiz var. americana (A. Gray) Fern.

Rare. One location. In dense growth of tall roadside plants.

TRT-Dys.

Lappula squarrosa (Retz.) Dumort

Prickly stickseed

L. echinata Gilib.

Rare. One location. Abandoned railroad tracks, on cinders and gravel. (+) TRT-Dys.

Lithospermum officinale $\mathrm{L}$.

Gromwell

Rare. Two locations. Dry, sandy waste area and open, damp ground beside swamp. (+)

TRT - Dys, Mnd.

Myosotis arvensis (L.) Hill

Forget-me-not

Uncommon. Damp ditches and open sandy areas. (+)

APM-Ngt; TRT - Hbn, Mnd.

Myosotis scorpioides L.

Forget-me-not

Rare. One location. Damp grassy area beside lake, on humus topsoil over sand. (+)

TRT - Dys.

Symphytum officinale $\mathbf{L}$.

Common comfrey

Rare. One location. Open cleared area, on disturbed soil near garden;

escape from cultivation. $(+)$

TRT-Mnd. 
Uncommon. Dry fields and roadsides, and moist meadows.

TRT-Hct, Hin, Mnd; S.R.-Lut.

Verbena simplex Lehm.

Narrow-leaf vervain

Rare. One location. Abandoned railroad tracks, in cinders and gravel. (+)

TRT-Sno.

LAMIACEAE

MINT FAMILY

(LABIATAE)

Galeopsis tetrahit L.

Hemp-nettle

Common. Moist roadsides, open fields, and sandy hillsides. (+)

APM-Ngt; TRT - Hbn, Hct, Mnd, Shb, Stn.

var. bifida (Boenn.) Lej. and Court.

Fairly common. Open waste ground and sandy roadsides. $(+)$

TRT-Dys, Gfd, Hbn, Mnd.

Glechoma hederacea $\mathbf{L}$.

Gill-over-the-ground

Uncommon. Moist edges of woods and driveways. (+)

TRT-Hct, Mnd; S.R.-Dys.

Leonurus cardiaca $\mathbf{L}$.

Motherwort

Uncommon. Sparsely treed area on clay soil, and open areas on borders of disturbed waste ground; on dry or moist soils. $(+)$

TRT-Dys, Mnd, Stn.

Lycopus americanus Muhlenb. ex Bartram

Cut-leaved water-horehound

Common. Damp to wet areas: ditches, flats, and borders of lakes and streams; usually on sandy soil.

TRT -Crd, Dys, Gfd, Hct, Mnd.

Lycopus uniflorus Michaux

Bugleweed

Common. On sandy shorelines of lakes, rivers, and creeks, and sometimes in muck in bogs and marshes.

TRT-Crd, Gfd, Hbn, Hct, Shb, Sno.

Mentha arvensis $\mathrm{L}$.

Wild mint

Common. Wet sandy shores, mud flats, and woodlands.

TRT-Gfd, Hbn, Hct, Shb, Stn. 
$=M$. aquatica $\mathrm{L} . \times M$. spicata $\mathrm{L}$.

Peppermint

Rare. One location. Grassy edge of sand beach. $(+)$

TRT-Stn.

\section{Monarda didyma $\mathrm{L}$.}

Oswego-tea

Rare. One location. In bramble thicket beside road; escape from

cultivation. $(+)$

TRT - Stn

\section{Monarda fistulosa $\mathrm{L}$.}

Wild bergamot

Rare. Two locations. Open hillside among tall grasses, and dry, disturbed open ground at entrance to bush road.

TRT-Crd, Mnd.

Nepeta cataria L.

Uncommon. Open, sandy waste sites. (+)

Catnip

TRT-Mnd.

\section{Prunella vulgaris $\mathrm{L}$.}

Common. Damp clearings in woods, and bush roads. (+)

TRT-Gfd, Hbn, Hin, Shb, Stn; S.R.-Ans, Cly, Glm, Sno.

Satureja acinos (L.) Scheele

Rare. One location. Abandoned railroad tracks, on crushed rock

ballast. $(+)$

TRT-Sno.

Satureja vulgaris (L.) Fritsch

Wild basil

Fairly common. Damp woods, dry or moist roadsides, and meadows. (+)

CAN-Crd; TRT -Hct, Stn; S.R.-Dys, Lut.

Scutellaria galericulata L.

Common skullcap

Common. Stream edges among grasses and sedges, and mud flats.

TRT-Crd, Gfd, Hct, Mnd; S.R._Liv.

Scutellaria lateriflora $\mathrm{L}$.

Mad-dog skullcap

Common. Wet shores, swampy areas in woods, and occasionally sandy roadsides.

TRT-Crd, Dys, Gfd, Hav, Hbn, Hct, Stn.

Stachys palustris $\mathrm{L}$.

Woundwort

var. pilosa (Nutt.) Fern.

Rare. Two locations. Old road in woods, and open, disturbed sandy ground of old garden.

TRT-Dys, Hct. 
Rare. One location. Edge of low shrubby bank above lake.

TRT-Dys.

\section{Thymus praecox Opiz}

subsp. arcticus (E. Durand) Jalas

T. serpyllum auct. non L.

Rare. One location. On open hillside and along path through woods; in large, often dense patches. (+)

TRT-Sno.

\section{SOLANACEAE}

Physalis heterophylla Nees

NIGHTSHADE FAMILY

Rare. Two locations. Sand-and-gravel banks in disturbed areas.

TRT-Dys, Sno.

Solanum dulcamara L.

Bittersweet nightshade

Uncommon. Edges of woods and disturbed sites. (+)

TRT-Gfd, Shb; S.R.-Crd.

Solanum nigrum $\mathbf{L}$.

Black nightshade

Uncommon. Sandy roadsides on edges of deciduous woods. (+)

TRT-Hbn, Mnd.

SCROPHULARIACEAE

Chaenorrhinum minus (L.) Lange

FIGWORT FAMILY

Dwarf snapdragon

Rare. One location. Abandoned railroad tracks, on rock ballast. (+)

TRT-Sno.

Chelone glabra $\mathrm{L}$.

Uncommon. Damp to wet shores, with low vegetation.

Turtlehead

TRT-Sno; S.R.-Sno.

Gratiola neglecta Torrey

Hedge-hyssop

Rare. Two locations. Wet sandbar, and damp depression on large, flat rock outcrop.

TRT-Hin, Mnd.

Linaria vulgaris Miller

Butter-and-eggs, Toadflax

Common. Open sandy and gravelly ground. (+)

TRT-Dys, Shb, Stn. 
Rare. One location. Low creek bank with sedges; in small dense colony partly in the water.

TRT-Crd.

Melampyrum lineare Desr.

Cow-wheat

Uncommon. Rocky, open or wooded shores.

TRT - Hin, Shb.

Mimulus ringens $\mathrm{L}$.

Monkey-flower

Fairly common. Wet ditches, river banks, and lake edges.

APM-Ngt; TRT-Crd, Dys, Hct; S.R.-Mnd, Sno.

Scrophularia lanceolata Pursh

Rare. One location. Sandy, open waste ground.

Hare figwort

TRT-Mnd.

Verbascum thapsus $\mathbf{L}$.

Common mullein

Common. Sandy waste ground, and dry, open roadsides. (+)

TRT_-Gfd, Hbn, Hct, Mnd, Shb; S.R.-Dys.

Veronica americana (Raf.) Schwein. ex Benth.

American brooklime

Uncommon. Edges of pools and streams, on muck or mud.

TRT-Crd, Hbn, Hct, Mnd.

Veronica anagallis-aquatica $\mathbf{L}$.

Water speedwell

Rare. One location. Cool, wet swampy ground beside spring.

TRT-Dys.

Veronica arvensis $\mathbf{L}$.

Corn speedwell

Rare. Two locations. Rocky bank in bed of moss, and dry sandy hillside.

$(+)$

TRT-Sno, Stn.

Veronica officinalis $\mathbf{L}$.

Common speedwell

Uncommon. Rocky ground in mixed woods, and thin deciduous

woods. (+)

TRT-Dys, Mon, Shb.

Veronica scutellata L.

Marsh speedwell

Common. Damp sandy shores of lakes and streams, wet rocky shores, and mud flats.

TRT-Crd, Hbn, Hct, Mnd, Shb. 
Common. Shorelines, open meadows, roadsides, and lawns. (+)

APM-Brt; TRT-Crd, Hct, Mnd; S.R.-Lut.

OROBANCHACEAE

Epifagus virginiana (L.) Barton

BROOM-RAPE FAMILY

Beechdrops

Rare. One location. Under tall beech trees in moist deciduous woods.

TRT-Dys.

LENTIBULARIACEAE

Utricularia cornuta Michaux

BLADDERWORT FAMILY

Horned bladderwort

Common. Mud flats, floating bog mats, and boggy shores.

TRT-Crd, Dys, Gfd, Glm, Hin, McC, Shb.

Utricularia geminiscapa Benj.

Twin bladderwort

Rare. One location. In quiet water $10 \mathrm{~cm}$ deep near shore of large pond; dense mass of underwater plants with 16 emersed flowering stems. Rare in Ontario (Argus et al., 1982-1987).

TRT-Shb.

Utricularia gibba L.

Humped bladderwort

Fairly common. Floating bog mats, and bog or muck shorelines; usually in small numbers, but in one swamp growing densely on sedge and grass hummocks and covering more than half a hectare.

TRT-Crd, Dys, Glm, Hin, McC.

Utricularia intermedia Hayne

Flat-leaf bladderwort

Fairly common. In large masses on boggy shorelines, and floating offshore in small shallow lakes and pools.

TRT-Dys, Hin, McC; S.R.-Mon, Shb.

Utricularia minor $\mathbf{L}$.

Northern bladderwort

Uncommon. In quiet, shallow stream backwaters, and sometimes creeping on wet flats and shores.

TRT-Crd, Glm, Lut, Mnd; S.R.-Dys.

Utricularia purpurea Walter

Purple bladderwort

Common. Small lakes and ponds; in floating masses in water $60 \mathrm{~cm}$ or more deep, flowering in shallower water on bog mats and mucky shores. DFB-Lwr, McC; TRT_Crd, Dys, Hin, Lut, Stn.

Utricularia resupinata B. D. Greene

Upside-down bladderwort ex Bigelow

Uncommon. Pools and shallow shorelines; on muck or soft mud.

APM-Lwr; TRT-Hbn, Shb. 
Common. Quiet or slow-moving waters of lakes, streams, and pools; free floating, often in large masses.

TRT-Crd, Dys, Hct, Lut, Mnd, Stn.

\section{PLANTAGINACEAE}

PLANTAIN FAMILY

\section{Littorella americana Fern.}
L. uniflora auct. non (L.) Ascherson

Rare. One location. Sand spits near entrance of river into lake.

CAN-Hav or Shb; MICH-Hav or Shb.

\section{Plantago lanceolata $\mathrm{L}$.}

Common. Open roadsides and dry, open grassy areas; often numerous. (+) APM-Ngt; TRT-Crd, Dys, Gfd, Hbn, Hin, Shb; S.R.-Mnd.

\section{Plantago major L.}

Common plantain

Common. Sandy roadsides and dry barren ground. (+)

TRT-Dys, Hct, Shb; S.R.-Mnd.

Plantago rugelii Decne.

Pale plantain

Rare. Two locations. Under trees and shrubs beside dried up swamp, and on roadside.

TRT-Crd, Hbn.

\section{RUBIACEAE}

Cephalanthus occidentalis $\mathrm{L}$.

Buttonbush

Uncommon. Edges of small lakes and rivers, in crowded shrub thickets.

TRT-Ans, Dys, Hin.

\section{Galium aparine L.}

Cleavers

Rare. One location. Dry grassy hilltop.

TRT-Hct.

\section{Galium asprellum Michaux}

Rough bedstraw

Uncommon. Edges of swamp pools and beside slow-moving creeks.

TRT-Hbn, Hct; S.R.-Hin.

\section{Galium boreale L.}

Rare. One location. Sandy roadside; in dense colony in strip $75 \mathrm{~m}$ long.

TRT-Dys.

Galium brevipes Fern. and Wieg.

Bedstraw Rare. Two locations. Sedge meadow beside stream, and wet clearing beside small lake.

TRT-Gfd, Shb; S.R.-Mnd. 
Rare. Two locations. Deciduous and mixed woods.

TRT-Hct, Lut.

Galium lanceolatum Torrey

Yellow wild licorice

Uncommon. Deciduous woods, on damp humus.

TRT-Crd, Dys; S.R.-Lut.

Galium mollugo L.

Bedstraw

Rare. One location. Open area on roadside, with grasses and sedges. (+) TRT-Stn.

Galium obtusum Bigelow

Bedstraw

Rare. One location. Open grassy area at edge of lake.

TRT-Dys.

Galium palustre L.

Bedstraw

Rare. One location. Wet sandy shore.

TRTE-Mnd.

Galium tinctorium L.

Bedstraw

Fairly common. Low, wet ditches, marshy areas, and edges of small streams.

TRT-Glm, Hct, Shb, Stn; S.R._-Hin.

Galium trifidum L.

Bedstraw

Uncommon. Wet edges of swamps, ponds, and creeks.

CAN-Crd; TRT-Crd, Dys, Shb; S.R.-Lut.

Galium triflorum Michaux

Fragrant bedstraw

Common. On roadsides and hillsides, in damp openings in woods, and beside streams; on sandy soils.

TRT-Dys, Gfd, Hct, Liv, Mon; S.R.-Crd.

Mitchella repens $\mathbf{L}$.

Partridge-berry

Common. Mixed or deciduous woods; on rich, moist soils or on drier knolls.

TRT_Crd, Dud, Gfd, Hct, Hin, McC, Mnd, Shb, Stn.

CAPRIFOLIACEAE

HONEYSUCKLE FAMILY

Diervilla lonicera Miller

Bush honeysuckle

Common. On rocky ridges and banks in partially wooded areas, and along bush roads; often in large colonies.

TRT-Gfd, Hbn, Hct, Mnd, Shb; S.R._Liv, Lut, Sno. 
Linnaea borealis $\mathbf{L}$.

Twinflower

Common. Mixed woods; creeping over damp, leaf-covered humus, or with mosses on rocks.

TRT-Dud, Dys, Gfd, Hbn, Hct, Shb; S.R.-Crd, Glm.

Lonicera $\times$ bella Zabel

$=$ L. tatarica L. $\times$ L. morrowil A. Gray

Pretty honeysuckle

Rare. One location. Sparse deciduous woods; introduced and well

established in woods near dwelling. (+)

TRT-Dys.

\section{Lonicera canadensis Bartram}

Fly honeysuckle

Fairly common. Openings in deciduous and mixed woods, on moist humus.

TRT-Hbn, Hct, Mnd.

\section{Lonicera dioica $\mathbf{L}$.}

Glaucous honeysuckle

Rare. Two locations. Mixed woods: on high rock ridge and in low, moist area.

TRT-Lut; S.R.-Mnd.

Lonicera hirsuta Eaton

Hairy honeysuckle

Rare. One location. Open, steep stony bank among low herbs and shrubs.

TRT-Mnd.

Lonicera tatarica $\mathbf{L}$.

Tartarian honeysuckle

Rare. One location. Old farm, on dry soil in fencerow. (+)

TRT-Mnd.

Sambucus canadensis $\mathbf{L}$.

Common elderberry

Uncommon. Damp hillside and wet edges of meadow and creek.

TRT-Gfd, Hct, Mnd.

Sambucus pubens Michaux

Red-berried elder

Common. Roadsides, hillsides, lake banks, and edges of mixed woods.

TRT-Crd, Gfd, Hct, Liv, Mnd; S.R.-Hbn, Hin.

Symphoricarpos albus (L.) S. F. Blake

Snowberry

Rare. One location. Partially wooded area on hilltop; in dense colony.

TRT-Mnd.

Symphoricarpos occidentalis Hook.

Wolfberry

Rare. One location. Dry grassy slope under sumac.

TRT-GIm. 
Rare. Two locations. Among scattered trees and shrubs on grassy edges of woods.

TRT-Dys; S.R.-Lut.

Viburnum acerifolium $\mathbf{L}$. Maple-leaved viburnum

Uncommon. Moist deciduous woods on rich humus, and thin mixed woods.

TRT-Lut, Mnd; S.R.-Lut.

\section{Viburnum alnifolium Marshall}

Hobble-bush

V. lantanoides Michaux

Common. Along roadsides in thickets, and on edges of woods; also found as nonblooming understory in tall woods.

DFB-Lwr; TRT-Dud, Gfd, Hct, Hin, Mnd.

\section{Viburnum cassinoides $\mathbf{L}$.}

Wild-raisin

Common. Wet thickets and low banks of lakes and streams.

TRT-Crd, Dys, Hct, Hin, Mnd, Shb, Stn.

\section{Viburnum lentago $\mathrm{L}$.}

Nannyberry

Uncommon. Banks and river edges with other tall shrubs, and low, wet meadows.

TRT-Dys, Gfd, Hin, Mnd; S.R.-Lut.

\section{Viburnum trilobum Marshall}

$V$. opulus L. var. americanum Aiton

Fairly common. Low, wet meadows and stream edges.

TRT-Crd, Hct, Mnd; S.R.-Dys, Lut, Sno.

\section{VALERIANACEAE}

VALERIAN FAMILY

\section{Valeriana officinalis $\mathbf{L}$.}

High-bush cranberry

Rare. Two locations. Areas of disturbed ground; escapes from cultivation. (+)

TRT-Dys; S.R.-Sno.

\section{CUCURBITACEAE}

Echinocystis lobata (Michaux) Torrey and A. Gray

Rare. Two locations. Dry and moist waste sites.

TRT-Dys, Shb. 
Uncommon. Marshy edges of slow-moving streams; partly reclining on sedges and grasses.

TRT-Hct, Mnd.

\section{Campanula rapunculoides $\mathbf{L}$.}

Creeping bellflower

Uncommon. Banks and grassy roadsides. (+)

TRT-Dys, Stn.

\section{LOBELIACEAE}

\section{Lobelia cardinalis $\mathrm{L}$.}

LOBELIA FAMILY

Cardinal-flower

Uncommon. Banks and wet edges of rivers and streams.

TRTE-McC; TRT-Mnd; S.R.-Hbn, Hin, Sno.

\section{Lobelia dortmanna $\mathbf{L}$.}

Water lobelia

Common. Shallow water near shore in small lakes; on sandy bottoms, usually half emersed, but once seen flowering vigorously under water in a season of high water level.

CAN-Crd; TRT-Cly, Crd, Hbn, Hct, Hin, Shb; TRTE-Stn.

\section{Lobelia inflata L.}

Indian-tobacco

Common. Moist or dry roadsides, mixed forests, and open rocky terrain. DFB-Lwr; TRT_Gfd, Hbn, Hct, Hin, Mnd, Shb.

Lobelia spicata Lam.

Pale-spike lobelia Rare. One location. High grassy hill sparsely covered with trees and shrubs. TRT-Dys.

ASTERACEAE

ASTER FAMILY

\section{(COMPOSITAE)}

Achillea millefolium $\mathrm{L}$.

Yarrow

Common. Roadsides, old fields, and disturbed sites; on dry sandy soils.

TRT-Crd, Dys, Gfd, Hct, Hin, Stn; S.R.-Ans, Sno.

Ambrosia artemisiifolia $\mathbf{L}$.

Ragweed

Common. Roadsides, in sand and gravel.

TRT-Dys; S.R.-Crd, Lut, Mnd.

Anaphalis margaritacea (L.) Benth. and Hook. f. ex C. B. Clarke

Common. Roadsides, sand flats, old fields, and damp ditches.

TRT-Crd, Gfd, Hct, Hin, Shb, Stn; S.R.-Brt, Cly, Glm. 
Fairly common. Dry roadsides, sandy slopes, meadows, and grassy soil pockets on rocks.

TRT-Dys, Hbn, Hct, Stn.

Antennaria plantaginifolia (L.) Richardson

Pussytoes

Rare. One location. Dry open woodland.

APM-Ngt.

Anthemis cotula $\mathrm{L}$.

Mayweed

Uncommon. Roadsides, in sand and gravel. (+)

TRT-Dys, Mnd, Sno.

Arctium minus (Hill) Bernh.

Common burdock

Fairly common. Open banks, roadsides, and borders of paths. (+)

TRT-Hct, Mnd, Shb, Stn.

Artemisia absinthium L.

Wormwood

Rare. One location. Sandy waste ground. (+)

TRT-Dys.

Artemisia ludoviciana Nutt.

var. gnaphalodes (Nutt.) Torrey and A. Gray

Mugwort

Uncommon. Sandy banks and dry open woods.

TRT - Dys, Hbn, Hct; S.R.-Shb.

Artemisia vulgaris $\mathrm{L}$.

Mugwort

Uncommon. Roadsides and waste areas. (+)

APM-Ngt; TRT-Dys.

Aster acuminatus Michaux

Acuminate aster

Rare. Two locations. In a dense colony of more than six hundred plants, many nonflowering, in a damp sugar maple wood, and numerous in a sparse mixed wood.

TRT-Dys, Shb.

Aster ciliolatus Lindley

Rare. Two locations. Rocky wooded areas.

Fringed blue aster

TRT_Lut, Shb.

Aster cordifolius $\mathbf{L}$.

Heart-leaved aster

Common. Deciduous or mixed open woodlands, and open roadsides; on dry or damp soils.

TRT-Crd, Gfd, Hbn, Hct, Hin, McC, Mnd, Shb. 
Rare. One location. Open, sandy, grassy flat beside road.

TRT-Sno.

Aster lateriflorus (L.) Britton

Calico aster

Uncommon. Damp roadsides, river banks, and partially shaded sandy areas.

TRT-Crd, Lut, Sno, Stn.

Aster macrophyllus L.

Large-leaved aster

Common. Wooded roadsides and edges of woods, on sand or humus; in large colonies, flowering sparsely.

TRT-Crd, McC, Mnd, Shb, Stn; S.R.-Cly.

\section{Aster nemoralis Aiton}

Bog aster

Fairly common. Wet, boggy or marshy shores of ponds and lakes; often among shrubs or cat-tails.

DFB-Lwr; APM-Lwr; TRT-Dys, Gfd, McC, Shb.

Aster novae-angliae $\mathbf{L}$.

New England aster

Uncommon. Open waste areas and openings in woods.

TRT-Hbn, Hct, Sno; S.R.-Ans.

Aster puniceus L.

Purple-stemmed aster

Fairly common. Wet ditches, swamp edges, and open damp areas.

TRT-Brt, Hct, Mnd, Shb; S.R.-Ans, Glm, Lut, Mon, Sno.

Aster simplex Willd.

Tall white aster

A. lanceolatus Willd.

Common. Roadsides, depressions in woods, swamp edges, and semi-open, flat sandy sites.

TRT -Crd, Dys, Lut, Mnd, Mon, Shb; S.R.-Ans, Sno.

\section{Aster umbellatus Miller}

Flat-topped aster

Common. Moist roadsides, open meadows, clearings, and shorelines; numerous across the county, often in large colonies.

TRT-Crd, Gfd, Hct, Hin, Lwr, McC, Shb, Stn; S.R.-Mon.

\section{Bidens beckii Torrey}

Water-marigold

Fairly common. Quiet water up to $60 \mathrm{~cm}$ deep in streams, bays, and lake margins; on sand or mud bottoms; flowers and upper leaves emersed. TRT-Crd, Hct, Mnd; S.R.-Dud, Dys. 
var. cernua

Common. Low marshy or sandy edges of streams and lakes, damp ditches and wet meadows.

TRT -Hct, Mnd, Stn; S.R.-Hin, Shb, Sno.

\section{var. minima (Huds.) Pursh}

Uncommon. Wet mud or sand flats, and wet lake edges.

TRT-Dys, Shb; S.R.-Glm.

Bidens comosa (A. Gray) Wieg.

Leafy-bracted beggar's-ticks

Rare. One location. Wet shore, on sphagnum moss.

TRT-Shb.

Bidens connata Muhlenb. ex Willd.

Beggar's-ticks

Uncommon. Wet shores and mossy logs at shorelines.

TRT-Crd, Hin; S.R.-Shb.

Bidens discoidea (Torrey and A. Gray) Britton

Beggar's-ticks Rare. Two locations. Edge of lake, and growing on partly emerged decaying logs in small creek. Rare in Ontario and in Canada (Argus et al., 19821987).

TRT-Crd, Mnd.

Bidens frondosa $\mathrm{L}$.

Beggar's-ticks

Fairly common. Dry or damp waste areas, wet hollows, and sand flats.

TRT-Dys, Gfd, Hbn, Shb, Stn; S.R.-Glm.

Bidens tripartita $\mathbf{L}$.

Beggar's-ticks

Uncommon. Boggy shores, and swampy places in woods.

TRT-Crd, Mnd, Shb; S.R.-Dys.

Bidens vulgata E. Greene

Rare. Two locations. Dry sandy roadsides.

Beggar's-ticks

TRT-Dys; S.R.-Mnd.

Centaurea maculosa Lam.

Spotted knapweed

Rare. Two locations. Edge of highway in sand and gravel, and dry sandand-grass slope. $(+)$

TRT-Cly, Sno.

Chrysanthemum leucanthemum $\mathrm{L}$.

Ox-eye daisy

Common. Roadsides, dry open banks, open wastelands, and old fields.

Usually on dry soils. (+)

TRT-Dys, Gfd, Hct, Mnd, Shb; S.R.-Crd, Mon. 
Fairly common. Dry roadsides and old fields; numerous and scattered. $(+)$ TRT-Dys, Shb, Stn.

Cirsium arvense (L.) Scop.

Canada thistle

Common. Dry and sandy, or moist and grassy roadsides. (+)

TRT-Crd, Dys; S.R.-Brt, Cly, Mon.

Cirsium vulgare (Savi) Ten.

Bull thistle

Common. Roadsides, old farmlands, and sand flats; soils various. (+)

APM-Cly; TRT-Hct, Mnd, Stn; S.R.-Dys, Mon.

Conyza canadensis (L.) Cronq.

Horseweed

Erigeron canadensis A. Gray

Fairly common. Sand flats, disturbed ground, and roadsides; often in large colonies.

APM-Ngt; TRT-Crd, Dys, Hct; S.R.-Glm.

Coreopsis grandiflora Hogg ex Sweet

Tickseed

Rare. One location. One clump in roadside ditch; escape from cultivation. (+)

TRT-Sno.

Crepis tectorum $\mathrm{L}$.

Narrow-leaved hawk's beard

Rare. One location. Open sand flat. (+)

TRT-Dys.

Erechtites hieracifolia (L.) Raf. ex DC.

Pilewort

Uncommon. Dry or damp waste ground and sandy and gravelly roadsides. TRT-Dys, Gfd, Hct, Hin, Shb.

Erigeron annuus (L.) Pers.

Daisy fieabane

Fairly common. Open hillsides, sparse woods, and roadside ditches.

TRT-Crd, Dud, Hct, Mnd, Mon; S.R.-Ans, Sno.

Erigeron philadelphicus $\mathrm{L}$.

Common fleabane

Fairly common. Borders of woods, paths and roadsides, and shady lake banks.

TRT -Dys, Gfd, Hct, Stn; S.R._Lut.

Erigeron strigosus Muhlenb. ex Willd.

Rough daisy fleabane

Uncommon. Open, sandy and rocky sites.

TRT-Dys, Gfd, Hct, Hin, Stn; S.R. - Lut. 
Common. Low, damp or wet areas beside streams, ponds, and marshes, and wet roadside ditches.

TRT_Crd, Dud, Hct, Hin, Mnd, Shb; S.R.-Brt, Cly.

Eupatorium perfoliatum $\mathrm{L}$.

Boneset

Common. Damp areas including roadside ditches, stream banks, swamp edges, and lakeshores.

TRT-Crd, Dys, Hct, Shb; S.R.-Mnd, Stn.

Gnaphalium sylvaticum $\mathrm{L}$.

Cudweed

Uncommon. Dry sandy roadsides, gravel pits, and lakeshores; on open sites or in partial shade.

TRT-Brt, Hbn, Hct, Hin.

Gnaphalium uliginosum $\mathrm{L}$.

Low cudweed

Uncommon. Sandy or muddy stream banks and damp lowlands.

TRT - Hct, Sno, Stn.

Gnaphalium viscosum Kunth

Cudweed

Rare. Two locations. Sparsely wooded bank and edge of bush road.

TRT-Gfd, Hin.

Helianthus annuus $\mathbf{L}$.

Common sunflower

Rare. Two locations. Open, sandy grassy roadsides; escapes from cultivation.

TRT-Dys, Stn.

Helianthus strumosus $\mathbf{L}$.

Woodland sunflower

Rare. Two locations. Damp clearings, with numerous low and tall herbs.

TRT-Gfd, McC.

Helianthus tuberosus $\mathrm{L}$.

Jerusalem artichoke

Rare. Two locations. In dense colony in roadside ditch, and scattered on waste ground. (+)

TRT-2 Dys.

Heliopsis helianthoides (L.) Sweet

Rough ox-eye

H. scabra Dunal

Rare. One location. Old estate land, 10 to 20 plants.

APM-Ngt.

Hieracium aurantiacum $\mathrm{L}$.

Orange hawkweed

Common. Fields, meadows, roadsides, and lakeshores. (+)

APM-Ngt; TRT_Crd, Gfd, Hct, Mnd, Shb; S.R.-Ans, Lut. 
Rare. Rocky sites, on shallow soil.

TRT-Hbn, Shb.

Hieracium $\times$ floribundum Wimmer and Grab.

Hybrid hawkweed

$=H$. caespitosum Dumort. $\times$ H. lactucella Wallr.

Uncommon. Roadside ditches and shady banks; on sand or clay. $(+)$

TRT-Crd, Hct, Mnd.

Hieracium pilosella $\mathbf{L}$.

Mouse-ear hawkweed

Uncommon. Soil pocket on rock, wet sedge-and-grass border of lake, and open site in patch of low grass; plants few in number. $(+)$

TRT - 2 Hin, Stn.

Hieracium piloselloides Villars

Yellow hawkweed

H. florentinum All.

Uncommon. Waste areas and lawns. (+)

TRT-Dys, Sno; S.R.-Crd.

Hieracium pratense Tausch

Uncommon. Dry banks, dry sand flats, and disturbed ground. (+)

TRT-Hbn, Shb, Stn; S.R.-Lut.

Hieracium scabrum Michaux

Rough hawkweed

Common. Roadsides and gravel pits; on sandy soils.

TRT-Dys, Gfd, Hbn, Hct, McC.

Lactuca biennis (Moench) Fern.

Wild lettuce

Fairly common. Roadside ditches, damp edges of woods, and dry, open sandy sites. Plants up to $4.3 \mathrm{~m}$ tall.

TRT-Gfd, Hct, Hin, McC, Mnd, Stn; S.R.-Dys, Sno.

Lactuca canadensis $\mathbf{L}$.

Wild lettuce var. latifolia Kuntze

Rare. One location. Edge of woods adjacent to open mown lawn.

TRT-Mnd.

Lactuca canadensis $\mathrm{L}$.

Wild lettuce

var. longifolia (Michaux) Farw.

Rare. Two locations. In clearing, and with tall herbs on roadside.

TRT-Dys, Hbn.

Lactuca hirsuta Muhlenb. ex Nutt.

Hairy lettuce

Rare. One location. Dry rocky point, on sparse soil.

TRT-Mnd. 
Rare. One location. Dry disturbed ground adjacent to parking lot. (+)

TRT-Mnd.

\section{Leontodon autumnalis $\mathrm{L}$.}

Fall dandelion

Rare. One location. Grassy ditch beside highway. (+)

TRT-Dys.

Matricaria matricarioides (Less.) Porter

Pineapple-weed

Common. Edges of dry roadsides and driveways; usually in large colonies. $(+)$

TRT-Dys, Stn; S.R.-Dys, Lut, Mnd, Sno.

Petasites frigidus (L.) Fries

Sweet coltsfoot

var. palmatus (Aiton) Cronq.

P. palmatus (Aiton) A. Gray

Rare. One location. Clearing in cedar-hemlock swamp.

TRT-Mnd.

Prenanthes alba L.

White lettuce

Rare. One location. Steep, clay river bank.

TRT-Sno.

Prenanthes altissima $\mathbf{L}$.

Tall white lettuce

Fairly common. Dry roadsides, and damp roadways through woods.

TRT-Crd, Gfd, Hbn, Hct, Hin, Stn.

Rudbeckia hirta L.

Black-eyed susan

Common. Fields, sandy roadsides, disturbed ground, and occasionally damp edges of woods.

TRT-Crd, Dys, Hct, Mnd, Shb, Stn.

Rudbeckia laciniata $\mathbf{L}$.

Green-headed coneflower

Rare. One location. Edge of woods on roadside. (+)

TRT-Hbn.

Rudbeckia serotina Nutt.

Black-eyed susan

$R$. hirta var. pulcherrima Farw.

Fairly common. Open fields, damp or dry roadsides, and edges of woods. (+)

TRT-Dys, Hct, Mnd, Shb, Stn. 
Uncommon. Grassy meadows in sandy loam, and edge of grassy area under shrubs.

TRT-Dys, Mnd; S.R.-Lut.

Solidago canadensis $\mathbf{L}$.

Canada goldenrod

Common. Sandy roadsides, and edges of woods and fields.

TRT_Crd, Gfd, Mnd, Shb; S.R._Ans, Glm.

Solidago graminifolia (L.) Salisb.

Grass-leaved goldenrod

Euthamia graminifolia (L.) Nutt.

Common. Meadows, fields, and edges of roads and woods; on dry or damp sandy soil.

TRT - Brt, Crd, Gfd, Hbn, Hct, Mnd, Shb.

Solidago hispida Muhlenb.

Hairy goldenrod

Uncommon. Dry or damp edges of woods, and roadside ditches.

TRT-Gfd, Hin, Shb.

Solidago juncea Aiton

Early goldenrod

Uncommon. Dry sandy edges of woods, and roadsides.

TRT - Gfd, Hct, Shb.

Solidago nemoralis Aiton

Gray goldenrod

Fairly common. Sandy roadsides and banks, and dry, grassy sand flats.

TRT-Crd, Dys, Mnd, Stn; S.R.-Ans, GIm.

Solidago rugosa Aiton

Rough-stemmed goldenrod

Common. Roadside ditches, open flats, and low banks; on dry or damp sandy soils.

TRT-Crd, Gfd, Hbn, Hct, Hin, McC, Mnd, Shb.

Solidago squarrosa Muhlenb. ex Nutt.

Stout goldenrod

Uncommon. Sandy roadsides and edges of woods.

TRT - Crd, McC, Mnd.

Solidago uliginosa Nutt.

Bog goldenrod

Common. Damp ditches and roadside depressions, with grasses and sedges; and edges of ponds, streams, bogs, and swamps.

TRT - Brt, Crd, Dys, Hct, Hin, Mnd, Shb.

Sonchus arvensis $\mathrm{L}$.

Field sow-thistle

Uncommon. Damp meadows and edges of woods. (+)

TRT-Hct, Dys; S.R.-Lut. 
Uncommon. Grassy open sites and weedy roadsides. (+)

TRT-Dys, Gfd; S.R.-Mnd.

Sonchus oleraceus L.

Common sow-thistle

Rare. Two locations. Dry roadside, and open grass area in town park. (+) TRT-Dys, Stn.

Sonchus uliginosus M. Bieb.

Sow-thistle

Rare. One location. Dry sandy border of highway. (+)

TRT-Gfd.

Tanacetum vulgare $\mathrm{L}$.

Tansy

Rare. Two locations. Low sandy bank and sparsely treed hilltop. (+)

TRT-Sno, Stn.

Taraxacum laevigatum (Willd.) DC.

Red-seeded dandelion

Rare. Two locations. Dry road edges, in sand and gravel. (+)

TRT-Mnd; S.R.-Dys.

Taraxacum officinale G. Weber

Common dandelion

Common. Open waste spaces of sparse vegetation and poor soil. $(+)$

TRT - Lut, Shb, Stn.

Tragopogon dubius Scop.

Goatsbeard

Uncommon. Dry hillsides and open grassy roadsides. (+)

TRT-Dys, Stn; S.R.-Ans.

Tussilago farfara $\mathbf{L}$.

Coltsfoot

Uncommon. Dry gravelly roadsides and sandy banks; usually in small dense colonies. $(+)$

TRT-Crd, Hav, Hct, Shb; S.R.-Mnd. 


\section{ACKNOWLEDGEMENTS}

This list of plants of the county of Haliburton could not have been produced without the assistance of the many persons who generously contributed their time and expertise to the project. We gratefully thank those named below and all the others who gave support in various ways.

Dale Hoy provided the original inspiration and support for the project, and Sheila McKay-Kuja gave much needed guidance during its progress, as well as assistance with difficult identifications.

The wide experience and botanical insights of R. Emerson Whiting were important contributions to many field trips and discussions. Paul Catling, Anton Reznicek, and John Riley provided valuable aid in resolving taxonomic and collecting problems. Steve Varga's field assistance also added significant data.

K. A. Armson contributed the information in the section on soils. J. L. Riley reviewed the section on wetlands, D. A. Metsger contributed the section on climate, and Celia Godkin produced the final rendition of the maps.

We are indebted to the experts who checked specimens for identification and nomenclature: G. W. Argus (Salix); P. W. Ball, W. J. Crins, and J. M. Webber (Carex); J. E. Eckenwalder (Populus); D. M. Britton (Dryopteris and Cystopteris); S. M. McKay-Kuja (Amelanchier); J. B. Phipps and T. A. Dickinson (Crataegus); P. M. Catling and R. E. Whiting (Orchidaceae).

The following collectors provided lists and specimens for specific areas of the county: D. Brunton, V. Connolly, L. Dickson, D. Lahaie, H. and R. Simpson, A. Whillans, and R. E. Whiting. Our thanks to them and to all other collectors who had the wisdom to place their specimens in herbaria so that other botanists could use the valuable information they represent.

We particularly thank Anton Reznicek for reviewing an early draft of the manuscript and providing many highly valued suggestions. The comments of two anonymous reviewers further improved the manuscript. Peter Ball helped proofread the manuscript and suggested several corrections and improvements.

Special thanks are owing to the Department of Botany, Royal Ontario Museum: to J. H. McAndrews, curator, for initially providing the support and facilities necessary for the production of the manuscript, as well as personally drafting the maps that appear as Figs. 2 and 3. Likewise we are indebted to T. A. Dickinson, assistant curator-in-charge, for revicwing the manuscript and for giving valuable advice and much practical assistance; to D. A. Metsger, curatorial assistant, for reviewing the manuscript and for vigorously tracking down needed data and references; to J. M. Ross, secretary, for her hours and hours of typing, proofreading, and indexing; and to E. Hamilton, volunteer, for help in preparing the indexes. 



\section{LITERATURE CITED}

APIOS (ACIDIC PRECIPITATION IN ONTARIO STUDY)

1981 Acid sensitivity survey of lakes in Ontario. Ontario Ministry of the

Environment. APIOS Report 002/81:1-30.

ARGUS, G. W., K. M. PRYOR, D. J. WHITE, and C. J. KEDDY, eds.

1982 - Atlas of the rare vascular plants of Ontario. Four parts. Ottawa, National

1987 Museum of Natural Sciences (looseleaf).

BRITTON, D. M.

1984 Checklist of Ontario pteridophytes. Part one: fern allies. Plant Press 2(4):95-99.

1985 Checklist of Ontario pteridophytes. Part two: ferns. Plant Press 3(1):14-23.

BROWN, D. M., G. A. MCKAY, and L. J. CHAPMAN

1974 The climate of southern Ontario. Environment Canada, Climatological Studies 5:1-50.

CHAPMAN, L. J.

1975 The physiography of the Georgian Bay-Ottawa Valley area of southern Ontario. Ontario Division of Mines, Geoscience Report 128:1-33.

DORE, W. G. and J. MCNEILL

1980 Grasses of Ontario. Agriculture Canada, Research Branch, Monograph 26:1-566.

DOUGLAS, R. J. W., ed.

1970 Geology and economic minerals of Canada. Geological Survey of Canada, Economic Geology Report 1:1-838.

FERNALD, M. L.

1950 Gray's manual of botany. 8th ed. New York, American Book. 1632 pp. GLEASON, H. A.

1952 The new Britton and Brown illustrated flora of the northeastern United States and adjacent Canada. New York, Hafner Publishing.

3 vols. $1731 \mathrm{pp}$.

GLEASON, H. A. and A. CRONQUIST

1963 Manual of vascular plants of northeastern United States and adjacent Canada. Toronto, Van Nostrand Reinhold. $810 \mathrm{pp}$.

GOUDGE, M. F.

1938 Limestones of Canada. Part IV. Ontario. Canada Department of Mines and Resources, Mines and Geology Branch, Publication 781:1-362.

HOLMGREN, P. K. and W. KEUKEN

1974 Index herbariorum. Part I. The herbaria of the world. 6th ed. Regnum Vegetabile 92:1-397.

JEGLUM, J. K., A. N. BOISSONNEAU, and F. V. HAAVISTO

1974 Toward a wetland classification for Ontario. Canadian Forestry Service, Information Report 0-X-215:1-54.

LUER, C. A.

1975 The native orchids of the United States and Canada, excluding Florida.

New York, New York Botanical Garden. 360 pp. 
MORTON, J. K. and J. M. VENN

1989 A synonymized checklist of the flora of Ontario-vascular plants (second draft August 1989). Waterloo, Ontario, by the authors. 153 pp.

PRINGLE, J. S.

1980 An introduction to wetland classification in the Great Lakes region.

Royal Botanical Gardens, Technical Bulletin 10:1-11.

REYNOLDS, N.

1968 In quest of yesterday. Lindsay, Provisional County of Haliburton. $343 \mathrm{pp}$.

ROWE, J.S.

1972 Forest regions of Canada. Canadian Forestry Service, Publication 1300:1-172.

SELL, P. D. and C. WEST

1976 Hieracium. In Tutin, T. G., et al., eds., Flora Europaea. Cambridge,

Cambridge University Press 4:358-410.

SOPER, J. H. and M. L. HEIMBURGER

1982 The shrubs of Ontario. Life Sciences Miscellaneous Publication.

Toronto, Royal Ontario Museum. 495 pp.

STAFLEU, F. A., C. E. B. BONNER, R. McVAUGH, R. D. MEIKLE, R. C. ROLLINS, R. ROSS,

J. M. SCHOPF, G. M. SCHULZE, R. de VILMORIN, and E. G. VOSS, eds.

1972 International code of botanical nomenclature adopted by the Eleventh

International Botanical Congress, Seattle, August 1969. Regnum

Vegetabile 82:1-426.

VOSS, E. G.

1972 Michigan flora. Part I. Gymnosperms and monocots. Cranbrook Institute of Science, Bulletin 55:1-488.

1985 Michigan flora. Part II. Dicots (Saururaceae-Cornaceae). Cranbrook Institute of Science, Bulletin 59:1-724.

WEBBER, J. M. and P. W. BALL

1984 The taxonomy of the Carex rosea group (section Thaestoglochin) in

Canada. Canadian Journal of Botany 62:2058-2073.

WHITING, R. E. and P. M. CATLING

1977 Distribution of the auricled twayblade orchid (Listera auriculata Wieg.) in Canada, and description of new stations in southern Ontario.

Canadian Field-Naturalist 91:403-406.

1986 Orchids of Ontario. Ottawa, CanaColl Foundation. 169 pp. 


\section{ADDITIONAL PUBLICATIONS CONSULTED IN IDENTIFICATION}

BEITEL, J.

1979 Clubmosses (Lycopodium) in North America. Bulletin of the American Fern Society 6(5):1-6.

BRITTON, D. M. and J. H. SOPER

1966 The cytology and distribution of Dryopteris species in Ontario.

Canadian Journal of Botany 44:63-78.

CASE, F. W. C.

1964 Orchids of the western Great Lakes Region. Cranbrook Institute of

Science, Bulletin 48:1-147.

CATLING, P. M. and J. E. CRUISE

1974 Spiranthes casei: a new species from northeastern North America.

Rhodora 76(808):526-536.

CINQ-MARS, L.

1966 Mise au point sur les violettes (Viola $\mathrm{spp}$.) du Québec. Naturaliste canadien 93:895-958.

CRINS, W. J.

1983 Nomenclature update of yellow Oxalis. Plant Press 1:14.

CROW, G. E. and C. B. HELLQUIST

1981 Aquatic vascular plants of New England. Part 2. Typhaceae and Sparganiaceae. New Hampshire Agricultural Experiment Station Bulletin 517:1-21.

1982 Aquatic vascular plants of New England. Part 4. Juncaginaceae, Scheuchzeriaceae, Butomaceae, Hydrocharitaceae. New Hampshire Agricultural Experiment Station Bulletin 520:1-20.

1983 Aquatic vascular plants of New England. Part 6. Trapaceae, Haloragaceae, Hippuridaceae. New Hampshire Agricultural Experiment Station Bulletin 524:1-26.

1985 Aquatic vascular plants of New England. Part 8. Lentibulariaceae. New Hampshire Agricultural Experiment Station Bulletin 528:1-22.

FASSETT, N. C.

1956 A manual of aquatic plants, rev. by E. C. Ogden. Madison, University of Wisconsin Press. 405 pp.

HAYNES, R. R.

1974 A revision of North American Potamogeton subsection Pusilli.

Rhodora 76(808):564-649.

HELLQUIST, C. B. and G. E. CROW

1980 Aquatic vascular plants of New England. Part 1. Zosteraceae, Potamogetonaceae, Zannicheliaceae, Najadaceae. New Hampshire Agricultural Experiment Station Bulletin 515:1-68.

1981 Aquatic vascular plants of New England. Part 3. Alismataceae. New Hampshire Agricultural Experiment Station Bulletin 518:1-32.

1982 Aquatic vascular plants of New England. Part 5. Araceae, Lemnaceac, Xyridaceae, Eriocaulaceae, and Pontederiaceac. New Hampshire Agricultural Experiment Station Bulletin 523:1-46. 
1984 Aquatic vascular plants of New England. Part 7. Cabombaceae, Nymphaeaceae, Nelumbonaceae, and Ceratophyllaceae.

New Hampshire Agricultural Experiment Station Bulletin 527:1-27.

HICKEY, J.

1977 The Lycopodium obscurum complex in North America. American Fern Journal 67:45-48.

HOSIE, R. C.

1973 Native trees of Canada. 7th ed. Ottawa, Canadian Forestry Service. $380 \mathrm{pp}$.

JONES, A. G. and P. HIEPKO

1981 The genus Aster s.l. (Asteraceae) in the Willdenow Herbarium at Berlin. Willdenowia 11:360-363.

MCKAY, S. M.

1973 A biosystematic study of the genus Amelanchier of Ontario. M.Sc. thesis, University of Toronto. $255 \mathrm{pp}$.

MORAN, R. C.

1983 Cystopteris tenuis (Michx.) Desv.: a poorly understood species.

Castanea 48:218-223.

MORTON, J. K.

1974 The giant cow parsnip Heracleum mantegazzianum in Canada.

Canadian Field-Naturalist 89:183-184.

PHIPPS, J. B. and M. MUNIYAMMA

1980 A taxonomic revision of Crataegus (Rosaceae) in Ontario. Canadian Journal of Botany 58:1621-1699.

PRINGLE, J. S.

1968 The common Solidago species (Goldenrods) of southern Ontario. Royal Botanical Gardens, Bulletin 3:1-14.

1976 The trilliums of Ontario. Royal Botanical Gardens, Bulletin 5:1-31.

1981 The common Aster species of southern Ontario. Royal Botanical Gardens, Bulletin 2:1-15.

REZNICEK, A. A. and R. S. BOBBETTE

1976 The taxonomy of Potamogeton subsection Hybridi in North America. Rhodora 78(816):650-672.

RILEY, J. L.

1979 Ontario's pondweeds (genus Potamogeton). Ontario Field Biologist $3(2): 1-26$.

RUSSELL, $N$.

1965 Violets of central and eastern United States. Sida 2(1):1 -111. SCOGGAN, H. J.

1978 - The flora of Canada. Ottawa, National Museums of Canada. 4 vol. 1979

SEMPLE, J. C. and G. S. RINGIUS

1983 The goldenrods of Ontario: Solidago L. and Euthamia Nutt.

University of Waterloo Biology Series 26:1-84.

SIMPSON, R. C. and P. M. CATLING

1978 A new natural hybrid orchid from Ontario. Canadian Field-Naturalist 92:350-358. 


\section{INDEX TO COMMON NAMES}

Adder's-mouth, green, 60

white, 12,60

ADDER'S-TONGUE FAMILY, 27

Agrimony, 77

Alder, speckled, 18, 63

Alfalfa, 84

Alyssum, 73

AMARANTH FAMILY, 67

AMARYLLIS FAMILY, 58

Anemone, Canada, 70

Apple, 80

Arrow-grass, 36

ARROW-GRASS FAMILY, 36

Arrowhead, 10, 36

northern, 36

Artichoke, Jerusalem, 116

ARUM FAMILY, 53

Ash, black, 17, 99

red, 99

white, 16, 99

Asparagus, 56

Aspen, large-toothed, 62

quaking, 62

Aster, acuminate, 112

bog, 113

calico, 113

flat-topped, 113

fringed blue, 112

heart-leaved, 112

heath, 113

large-leaved, 113

New England, 113

purple-stemmed, 113

tall white, 113

ASTER FAMILY, 111-120

Avens, 79

large-leaved, 79

purple, 79

white, 79

yellow, 79

Awlwort, 75

Balsam-fir, 16, 17, 31

Baneberry, red, 70 white, 70

BARBERRY FAMILY, 72

Basswood, 16, 88

BAYBERRY FAMILY, 63

Beak rush, 17, 52

BEAN FAMILY, 83-85

Bearberry, 97

Bedstraw, 107, 108

fragrant, 108

northern, 107

rough, 107

Beech, American, 16, 64

Beechdrops, 106

BEECH FAMILY, 64

Beggar's-ticks, 114

leafy-bracted, 114

nodding, 114

Bellflower, creeping, 111 marsh, 111

Bellwort, large-flowered, 57

Bindweed, 65

black, 65

field, 100

hedge, 100

Birch, paper, 63

white, 63

yellow, 16, 63

BIRCH FAMILY, 63-64

Bird's-foot trefoil, 83

Bittersweet, 86

Black locust, 84

Black-eyed susan, 118

Blackberry, bristly, 82

common, 81

smooth, 81

Bladderwort, common, 107

flat-leaf, 106

horned, 106

humped, 106

northern, 106

purple, 106

twin, 106

upside-down, 106

BLADDERWORT FAMILY, 106-107 
Bloodroot, 73

Blue-eyed grass, 58

Blueberry, low sweet, 98 velvet-leaf, 98

Bog-cotton, 52 tawny, 52

Bog-laurel, 17, 98

Bog-rosemary, 17, 97

Boneset, 116

BORAGE FAMILY, 101

Bouncing-bet, 68

Box-elder, 86

Brooklime, American, 105

BROOM-RAPE FAMILY, 106

Buckbean, 17, 99

Buckthorn, alder-leaved, 87

BUCKTHORN FAMILY, 87

BUCKWHEAT FAMILY, 65-66

Buffalo-berry, 91

Bugleweed, 102

Bulrush, 18, 52-53

hardstem, 52

soft-stem, 18, 53

water, 53

Bunchberry, 14, 96

Burdock, common, 112

Burnet-saxifrage, 95

Bur-reed, 18, 33

floating, 33

BUR-REED FAMILY, 33

Butter-and-eggs, 104

Buttercup, bristly, 72

creeping, 72

cursed, 72

hooked, 72

kidneyleaf, 71

tall, 71

Buttonbush, 107

Campion, bladder, 69 white, 68

Caraway, 94

Cardinal-flower, 111

Carpetweed, 67

CARPETWEED FAMILY, 67

CASHEW FAMILY, 86

Catchfly, garden, 68 night-flowering, 68 sleepy, 68

Catnip, 103

Cat-tail, common, 18, 32 narrow-leaved, 18,32

CAT-TAIL FAMILY, 32

Cedar, eastern white, 17, 32

Celandine, 73

Charlock, 74

Cherry, black, 81

choke, 81

pin, 80

Chickweed, common, 69

field, 67

mouse-ear, 67

Chicory, 19, 115

Chokeberry, 18, 78

Cinquefoil, 80

common, 80

marsh, 80

rough, 80

rough-fruited, 80

silvery, 80

Cleavers, 107

Clover, alsike, 84

hop, 84

red, 84

white, 84

white sweet, 84

yellow sweet, 84

Clubmoss, bog, 25

bristly, 25

running pine, 25

savin-leaved, 26

shining, 25

staghorn, 25

tree, 25

CLUBMOSS FAMILY, 25-26

Cohosh, blue, 72

Coltsfoot, 120 sweet, 118

Columbine, garden, 71 wild, 71

Comfrey, common, 101 wild, 101

Coneflower, green-headed, 118

Coontail, 19, 69

Coral-root, early, 18, 58 spotted, 58 
striped, 58

Corydalis, pale, 73

Cotton-grass, 12, 17, 52

Cow-wheat, 105

Cranberry, high-bush, 110

large, 17,98

small, 17, 98

Cranesbill, Bicknell's, 85

Cress, bitter, 17, 74

field, 15,75

marsh, 75

rock, 73

water, 75

winter, 73

CROWFOOT FAMILY, 70-72

Crown-vetch, 83

Cucumber-root, Indian, 56

Cudweed, 116

low, 116

Currant, bristly black, 77

red, 77

skunk, 77

swamp red, 18, 77

CYPRESS FAMILY, 32

Daisy, ox-eye, 114

Dandelion, common, 120

fall, 118

red-seeded, 120

Day-lily, orange, 56 yellow, 56

Dewberry, northern, 82 swamp, 82

Dewdrops, 79

Dock, bitter, 66

curly, 66

patience, 66

sour, 66

Dogbane, spreading, 100

DOGBANE FAMILY, 100

Dogwood, pagoda, 96

red osier, 96

round-leaved, 96

silky, 96

DOGWOOD FAMILY, 96

Doll's-eyes, 70

Duckweed, 18, 53

greater, 53
DUCKWEED FAMILY, 53

Dutchman's breeches, 16, 73

Elder, red-berried, 109

Elderberry, common, 109

Elm, rock, 64 white, 64

ELM FAMILY, 64

Enchanter's nightshade, 92 smaller, 18, 92

Evening primrose, 93 small-flowered, 93

EVENING PRIMROSE FAMILY, 92-93

Fern, bracken, 30

bulblet bladder, 28

Christmas, 30

cinnamon, 18, 27

crested shield, 29

evergreen wood, 29

fragrant wood, 29

Goldie's, 29

hay-scented, 29

hybrid, 29, 30

interrupted, 28

maidenhair, 28

marginal shield, 29

marsh, 31

New York, 30

northern fragile, 29

northern beech, 30

oak, 30

ostrich, 30

rattlesnake, 27

royal, 18,28

sensitive, 18,30

spinulose wood, 29

upland lady, 28

Virginia chain, 31

Fescue, hard, 39

meadow, 39

red, 39

Rocky Mountain, 39

tall, 39

Feverwort, 110

Figwort, hare, 105

FIGWORT FAMILY, 104- 106 
Fireweed, 19, 92

Flag, blue, 58

Fleabane, common, 115

daisy, 115

rough daisy, 115

Floating-heart, 100

Foamflower, 77

Forget-me-not, 101

FOUR-O'CLOCK FAMILY, 67

Foxtail, green, 43 short awn, 37 yellow, 43

FROG'S-BIT FAMILY, 36 FUMITORY FAMILY, 73

Galingale, 50

Gentian, narrow-leaved, 99

GENTIAN FAMILY, 99-100

GERANIUM FAMILY, 85

Germander, 104

Gill-over-the-ground, 102

Ginseng, 94

GINSENG FAMILY, 94

Goatsbeard, 120

Goldenrod, bog, 119

Canada, 119

early, 119

grass-leaved, 119

gray, 119

hairy, 119

rough-stemined, 119

stout, 119

Goldthread, 71

Gooseberry, prickly, 77 . wild, 77

Goosefoot, maple-leaved, 66

GOOSEFOOT FAMILY, 66-67

GOURD FAMILY, 110

Goutweed, 94

GRAPE FAMILY, 87

Grape fern, cut-leaved, 27

dwarf, 27

leathery, 27

matricary, 27

Grass, alkali, 43

annual blue, 42

autumn bent, 37

barnyard, 38 blue joint reed, 38

bottlebrush, 39

broadleaf panic, 41

Canada blue, 43

creeping bent, 37

cut, 40

downy brome, 38

English rye, 40

false medic, 43

floating manna, 18,39

fowl manna, 40

fowl meadow, 43

fringed brome, 37

hair, 38

Kentucky blue, 43

large crab, 38

leafy wood, 37

long manna, 40

moor mat, 41

muhly, 40-41

nodding wood, 38

northern reed, 38

orchard, 38

panic, 41,42

plume, 40

poa, 43

poverty, 38

quack, 36

rattlesnake, 18, 39

redtop, 37

reed, 42

reed canary, 42

ribbon, 42

rice, 41

smooth brome, 37

smooth crab, 38

sweet, 40

sweet vernal, 37

tall cord, 43

tall manna, 39

tickle, 37

velvet, 40

western barnyard, 38

wheat, 37

witch, 41, 42

wood blue, 43

yellow panic, 42

GRASS FAMILY, 36-44 
Grass-pink, 17, 58

Greenbrier, prickly, 57

Gromwell, 101

Ground-cedar, 25, 26

Ground-cherry, clammy, 104

Ground-pine, 25

HAREBELL FAMILY, 111

Hawk's beard, narrow-leaved, 115

Hawkweed, Canada, 117

hybrid, 117

mouse-ear, 117

orange, 116

rough, 117

yellow, 117

Hawthorn, 78-79

Hazel, beaked, 63

Heal-all, 103

HEATH FAMILY, 97-98

Hedge-hyssop, 10, 104

Heliotrope, garden, 110

Helleborine, 59

Hemlock, eastern, 16, 32

Hemp, Indian, 100

Hemp-nettle, 102

Hepatica, round-lobed, 71

sharp-leaved, 71

Herb-robert, 85

Herb-sophia, 74

Hobble-bush, 110

HOLLY FAMILY, 86

Honewort, 95

Honeysuckle, bush, 108

fly, 109

glaucous, 109

hairy, 109

pretty, 109

Tartarian, 109

HONEYSUCKLE FAMILY, 108-110

Hop, common, 64

HORNWORT FAMILY, 69

Horsetail, common, 26

variegated, 27

water, 18,26

woodland, 27

HORSETAIL FAMILY, 26-27

Horseweed, 115

Hound's-tongue, 101
Huckleberry, black, 98

Indian-pipe, 96

Indian-tobacco, 111

IRIS FAMILY, 58

Ironwood, 16, 64

Jack-in-the-pulpit, 53

Jewelweed, 18, 87

Joe-Pye weed, 116

Johnny-jump-up, 91

Juniper, common, 32

King devil, 117

Knapweed, spotted, 114

Knawel, 68

Knotweed, Japanese, 65 prostrate, 65

Labrador tea, 98

Ladies' tresses, Case's, 61 hooded, 61

hybrid, 61

nodding, 61

slender, 61

Lady's-slipper, large yellow, 59

pink, 58

queen, 59

showy, 59

Lady's-thumb, 66

Lamb's quarters, 66

Leatherleaf, 17, 97

Leatherwood, 91

Lettuce, hairy, 117

prickly, 118

tall white, 118

white, 118

wild, 117

Licorice, white wild, 108 yellow wild, 108

Lilac, common, 99

Lily, corn, 56

trout, 56

wood, 56

LILY FAMILY, 55-57

LINDEN FAMILY, 88

Littorella, 107

Live-forever, 76 
Lobelia, pale-spike, 111 water, 10,111

LOBELIA FAMILY, 111

Loosestrife, dotted, 99 fringed, 98

purple, 91

swamp, 91

tufted, 99

LOOSESTRIFE FAMILY, 91

Lychnis, scarlet, 68

MADDER FAMILY, 107-108

Mallow, common, 88 musk, 88

MALLOW FAMILY, 88

Maple, Manitoba, 86 mountain, 87

red, 17, 87

silver, 87

striped, 86

sugar, 15, 87

MAPLE FAMILY, 86-87

Mare's-tail, 94

MARE'S-TAIL FAMILY, 94

Marijuana, 64

Marsh-marigold, 71

Mayweed, 112

Meadow-beauty, 91

Meadow-rue, early, 72 purple, 72 tall, 72

Meadowsweet, broad-leaved, 83 narrow-leaved, 82

Medick, black, 83

MELASTOMA FAMILY, 91

Mermaid-weed, 94

MEZERUM FAMILY, 91

Mignonette, 75

MIGNONETTE FAMILY, 75

Milkweed, common, 100 swamp, 100

MILKWEED FAMILY, 100

Milkwort, 85

MILKWORT FAMILY, 85

Millet, broomcorn, 42 wood, 40

MINT FAMILY, 102-104

Mitrewort, 76 naked, 17,76

Moccasin flower, pink, 58

white-lipped, 59

Mock-orange, 76

Monkey-flower, 105

Monkshood, 70

MORNING-GLORY FAMILY, 100

Moss-pink, 101

Mother-of-thyme, 103

Motherwort, 102

Mountain-ash, 82

showy, 82

Mountain holly, 18, 86

Mugwort, 112

MULBERRY FAMILY, 64

Mullein, common, 105

Mustard, field, 74

hedge, 75

tower, 73

tumble, 75

wormseed, 74

MUSTARD FAMILY, 73-75

Naiad, 35

Nannyberry, 110

Narcissus, poet's, 58

Nettle, false, 64

stinging, 65

wood, 18, 64

NETTLE FAMILY, 64-65

Nightshade, bittersweet, 104 black, 104

NIGHTSHADE FAMILY, 104

Ninebark, 80

Oak, red, 64

OLEASTER FAMILY, 91

OLIVE FAMILY, 99

Orchid, blunt-leaf, 18, 60

club-spur, 60

green fringed, 60

large round-leaved, 60

long-bracted green, 58

purple fringed, 61

tall northern green, 18,60

ORCHID FAMILY, 58-61

ORPINE FAMILY, 76

Oswego-tea, 103 
Ox-eye, rough, 116

PARSLEY FAMILY, 94-95

Parsnip, cow, 95 giant cow, 95 water, 95 wild, 95

Partridge-berry, 14, 108

Pea, everlasting, 83 sweet, 83

Pearlwort, 68

Pearly everlasting, 111

Penny-cress, field, 75

Peppergrass, 75

Peppermint, 103

Periwinkle, 100

Phlox, 100

PHLOX FAMILY, 100-101

Pickerel-weed, 18, 54

PICKEREL-WEED FAMILY, 54

Pigweed, 67

Pilewort, 115

Pimpernel, false, 105

Pine, red, 16, 32

Scotch, 32

white, 16, 32

Pineapple-weed, 118

PINE FAMILY, 31-32

Pinesap, 96

Pink, Deptford, 67

garden, 68

maiden, 68

PINK FAMILY, 67-69

Pinweed, 89

Pipewort, 54

PIPEWORT FAMILY, 54

Pipsissewa, 96

Pitcher-plant, 17, 76

PITCHER-PLANT FAMILY, 76

Plantain, common, 15, 107

English, 107

pale, 107

PLANTAIN FAMILY, 107

Plum, Canada, 80

Poison ivy, 86

Polygala, fringed, 85

Polypody, common, 30

POLYPODY FAMILY, 28-31
Pondweed, 18, 19, 34, 35

alpine, 33

flat-stemmed, 35

floating, 34

large-leaf, 33

leafy, 34

narrow-leaved, 33-34, 35

sago, 34

snailseed, 35

variable, 34

PONDWEED FAMILY, 33-35

Poplar, balsam, 62

silver, 61

white, 61

POPPY FAMILY, 73

PRIMROSE FAMILY, 98-99

PURSLANE FAMILY, 67

Pussytoes, 112

field, 112

Pyrola, 96

green, 97

one-sided, 97

PYROLA FAMILY, 96-97

Queen Anne's-lace, 95

Queen-of-the-prairie, 79

Quillwort, 26

QUILLWORT FAMILY, 26

Ragweed, 111

Ragwort, balsam, 119

Raspberry, dwarf, 18, 82 purple-flowering, 82 wild red, 82

Rattlesnake-plantain, 59 dwarf, 59

Menzies', 59

Rocket, dame's, 74

ROCKROSE FAMILY, 89

Rose, many-flowered, 81 prickly wild, 81 smooth wild, 81 swamp, 81

ROSE FAMILY, 77-83

Rose-pogonia, 17, 61

ROYAL FERN FAMILY, 27-28

Rush, 15, 18, 54, 55

bayonet, 55 
Canada, 18, 54

needle, 51

path, 55

soft, 55

toad, 54

wood, 55

RUSH FAMILY, 54-55

Rye, 43

Rye-grass, Virginia wild, 39

SANDALWOOD FAMILY, 65

Sandwort, thyme-leaved, 67

Sarsaparilla, bristly, 94 wild, 94

Saxifrage, early, 14, 77

SAXIFRA GE FAMILY, 76-77

Scouring-rush, dwarf, 26 rough, 26

Sedge, 12, 15, 17, 18, 44-50

three-way, 51

umbrella, 50

SEDGE FAMILY, 44-53

Selfheal, 103

Serviceberry, 77, 78

smooth, 78

Sheep-laurel, 98

Sheep-sorrel, 15, 66

Shepherd's purse, 74

Shinleaf, 18, 97

Silverweed, 80

Skullcap, common, 103 mad-dog, 103

Smartweed, bristly, 65

common, 65

dotted, 66

pale, 66

water, 65

Snakeroot, black, 95

Snapdragon, dwarf, 104

Snowberry, 109 creeping, 97

Solomon's-seal, false, 56 hairy, 56 starry, 57 three-leaved, 17, 57

Sow-thistle, 120

common, 120

field, 119 spiny-leaved, 120

Spearwort, creeping, 15, 72

Speedwell, common, 105

corn, 105

marsh, 105

thyme-leaved, 106

water, 105

Spikemoss, rock, 26

SPIKEMOSS FAMILY, 26

Spikenard, 94

Spike-rush, 15, 18, 51

Spiraea, false, 82

Spleenwort, maidenhair, 28 narrow-leaved, 12, 28

silvery, 28

Spring-beauty, 16, 67

Spruce, black, 17, 31

red, 31

white, 31

Spurge, 85

cypress, 85

spotted, 86

sun, 85

SPURGE FAMILY, 85-86

Spurrey, corn, 69

Squirrel-corn, 16, 73

St. John's-wort, Canadian, 88 common, 89

dwarf, 88

large, 88

marsh, 89

northern, 88

pale, 88

ST. JOHN'S-WORT FAMILY, 88-89

STAFF-TREE FAMILY, 86

Starflower, 99

Starwort, 19, 69

Steeple-bush, 83

Stickseed, 101 prickly, 101

Stitchwort, lesser, 69

Stonecrop, mossy, 14, 76

Spanish, 76

Strawberry, barren, 83

wild, 79

wood, 79

Strawberry-blite, 66

Sumac, staghorn, 86 
Sundew, round-leaved, 17, 76 spatulate-leaved, 17, 76

SUNDEW FAMILY, 76

Sundrops, 93

Sunflower, common, 116 woodland, 116

Swamp-candles, 99

Sweet-brier, 81

Sweet cicely, 95

Sweet-fern, 63

Sweetflag, 53

Sweet gale, 17, 63

Tamarack, 17, 31

Tansy, 120

Tansy-mustard, 74

Tapegrass, 19, 36

Tear-thumb, 66

Thimbleweed, 70

Thistle, bull, 19, 115

Canada, 19, 115

Russian, 67

Tickseed, 115

Timothy, 42

Toadflax, 104 bastard, 65

Toothwort, 74

Touch-me-not, 87

TOUCH-ME-NOT FAMILY, 87

Trailing-arbutus, 97

Trillium, painted, 57

red, 57

red (yellow form), 57

white, 57

Turtlehead, 104

Twayblade, auricled, 59

Loesel's, 59

Twig-rush, 50

Twinflower, 14, 109

Twisted-stalk, rose, 57

Umbrellawort, 67

VALERIAN FAMILY, 110

Vervain, blue, 102 narrow-leaf, 102

VERVAIN FAMILY, 102

Vetch, cow, 84 four-sided, 85

narrow-leaved, 84

Vetchling, 83

yellow, 83

Viburnum, maple-leaved, 110

Violet, American dog, 89

blue marsh, 89

blue marsh (white form), 90

Canada, 89

downy yellow, 90

great-spurred, 90

hooked-spur, 89

kidney-leaved, 18, 90

lance-leaved, 10, 90

large-leaved white, 90

northern blue, 91

northern bog, 90

northern white, 90

smooth yellow, 90

sweet white, 89

woolly blue, 91

VIOLET FAMILY, 89-91

Viper's bugloss, 19, 101

Virginia creeper, 87

Virgin's-bower, 71

Wall-rocket, 74

Wartweed, 86

Water-arum, 18, 53

Water-carpet, golden, 76

Water-crowfoot, white, 71

yellow, 71

Water-hemlock, 94

Water-horehound, cut-leaved, 102

Water-lily, white, 18, 70

yellow, 18, 69, 70

WATER-LILY FAMILY, 69-70

Water-marigold, 113

Water-milfoil, 18, 19, 93

Eurasian, 93

WATER-MILFOIL FAMILY, 93-94

Water-pennywort, 95

Water-plaintain, 36

WATER-PLANTAIN FAMILY, 36

Water-purslane, 92

Water-shield, 18, 69

Water-starwort, 86

WATER-STARWORT FAMILY, 86 
Waterweed, 19, 36

Waterwort, 89

WATERWORT FAMILY, 89

Wheat, hard, 44

Wild basil, 103

Wild bergamot, 103

Wild calla, 53

Wild chive, 55

Wild cucumber, 110

Wild iris, 18, 58

Wild leek, 16, 55

Wild lily-of-the-valley, 56

Wild mint, 102

Wild-raisin, 18, 110

Wild thyme, 104

Willow, balsam, 63

beaked, 62

crack, 62

flat-leaved, 63

pussy, 62

shining, 62

slender, 63

stiff, 62 upland, 62

WILLOW FAMILY, 61-63

Willow-herb, 92

hairy, 92

narrow-leaved, 92

Winterberry, 18,86

Wintergreen, 98 one-flowered, 96

Wolfberry, 109

Woodsia, rusty, 31

Wood-sorrel, 85

yellow, 85

WOOD-SORREL FAMILY, 85

Wool-grass, 52

Wormwood, 112

Woundwort, 103

Yarrow, 111

Yellow-eyed grass, 53-54

YELLOW-EYED GRASS FAMILY, 53-54

Yew, American, 31

YEW FAMILY, 31 


\section{INDEX TO SCIENTIFIC NAMES}

Page numbers in italics indicate synonyms.

Abies, 16, 17, 31

Acer, 15, 17, 86-87

ACERACEAE, 86-87

Achillea, 111

Aconitum, 70

Acorus, 53

Actaea, 70

Adiantum, 28

Aegopodium, 94

Agrimonia, 77

Agropyron, 36-37

Agrostis, 37

AIZOACEAE, 67

Alisma, 36

ALISMATACEAE, 36

Allium, 16, 55

Alnus, 18, 63

Alopecurus, 37

Alyssum, 73

AMARANTHACEAE, 67

Amaranthus, 67

AMARYLLIDACEAE, 58

Ambrosia, 111

Amelanchier, 77-78

ANACARDIACEAE, 86

Anacharis, 36

Anaphalis, 111

Andromeda, 17, 97

Anemone, 70

Antennaria, 112

Anthemis, 112

Anthoxanthum, 37

APIACEAE, 94-95

APOCYNACEAE, 100

Apocynum, 100

AQUIFOLIACEAE, 86

Aquilegia, 71

Arabis, 73

ARACEAE, 53

Aralia, 94

ARALIACEAE, 94

Arctium, 112
Arctostaphylos, 97

Arenaria, 67

Arisaema, 53

Aronia, 18, 78

Artemisia, 112

ASCLEPIADACEAE, 100

Asclepias, 100

Asparagus, 56

Asplenium, 28

Aster, 112-113

ASTERACEAE, 111-120

Athyrium, 12, 28

BALSAMINACEAE, 87

Barbarea, 73

BERBERIDACEAE, 72

Betula, 16, 63

BETULACEAE, 63-64

Bidens, 113-114

Boehmeria, 64

BORAGINACEAE, 101

Botrychium, 27

Brachyelytrum, 37

Brasenia, 18, 69

Brassica, 74

BRASSICACEAE, 73-75

Bromus, 37-38

Calamagrostis, 38

Calla, 18, 53

CALLITRICHACEAE, 86

Callitriche, 19, 86

Calopogon, 17, 58

Caltha, 71

Campanula, 111

CAMPANULACEAE, 111

Cannabis, 64

CAPRIFOLIACEAE, 108-110

Capsella, 74

Cardamine, 17, 74

Carex, 12, 15, 17, 18, 44-50

Carum, 94 
CARYOPHYLLACEAE, 67-69

Caulophyllum, 72

CELASTRACEAE, 86

Celastrus, 86

Centaurea, 114

Cephalanthus, 107

Cerastium, 67

CERATOPHYLLACEAE, 69

Ceratophyllum, 19, 69

Chaenorrhinum, 104

Chamaedaphne, 17, 97

Chelidonium, 73

Chelone, 104

CHENOPODIACEAE, 66-67

Chenopodium, 66

Chimaphila, 96

Chiogenes, 97

Chrysanthemum, 114

Chrysosplenium, 76

Cichorium, 19, 115

Cicuta, 94

Cinna, 38

Circaea, 18, 92

Cirsium, 19, 115

CISTACEAE, 89

Cladium, 50

Claytonia, 16, 67

Clematis, 71

Clintonia, 56

Coeloglossum, 58

Comandra, 65

Comptonia, 63

COMPOSITAE, see ASTERACEAE

CONVOLVULACEAE, 100

Convolvulus, 100

Conyza, 115

Coptis, 71

Corallorhiza, 18, 58

Coreopsis, 115

CORNACEAE, 96

Cornus, 14, 96

Coronilla, 83

Corydalis, 73

Corylus, 63

CRASSULACEAE, 76

Crataegus, 78-79

Crepis, 115

CRUCIFERAE, see BRASSICACEAE
Cryptotaenia, 95

CUCURBITACEAE, 110

CUPRESSACEAE, 32

Cynoglossum, 101

CYPERACEAE, 44-53

Cyperus, 50

Cypripedium, 58-59

Cystopteris, 28-29

Dactylis, 38

Dalibarda, 79

Danthonia, 38

Daucus, 95

Decodon, 91

Dennstaedtia, 29

Dentaria, 74

Deschampsia, 38

Descurainia, 74

Dianthus, 67-68

Dicentra, 16, 73

Diervilla, 108

Digitaria, 38

Diplotaxis, 74

Dirca, 91

Drosera, 17, 76

DROSERACEAE, 76

Dryopteris, 29-30, 30

Dulichium, 51

Echinochloa, 38

Echinocystis, 110

Echium, 19, 101

ELATINACEAE, 89

Elatine, 89

ELAEAGNACEAE, 91

Eleocharis, 15, 18, 51

Elodea, 19, 36

Elymus, 39

Epifagus, 106

Epigaea, 97

Epilobium, 19, 92

Epipactis, 59

EQUISETACEAE, 26-27

Equisetum, 18, 26-27

Erechtites, 115

ERICACEAE, 97-98

Erigeron, 115, 115

ERIOCAULACEAE, 54 
Eriocaulon, 54

Eriophorum, 12, 17, 52

Erysimum, 74

Erythronium, 56

Eupatorium, 116

Euphorbia, 85-86

EUPHORBIACEAE, 85-86

Euthamia, 119

FABACEAE, 83-85

FAGACEAE, 64

Fagus, 16, 64

Festuca, 39

Filipendula, 79

Fragaria, 79

Fraxinus, 16, 17, 99

FUMARIACEAE, 73

Galeopsis, 102

Galium, 107-108

Gaultheria, 97-98

Gaylussacia, 98

Gentiana, 99

GENTIANACEAE, 99-100

GERANIACEAE, 85

Geranium, 85

Geum, 79

Glechoma, 102

Glyceria, 18, 39-40

Gnaphalium, 116

Goodyera, 59

GRAMINEAE, see POACEAE

Gratiola, 10, 104

Gymnocarpium, 30

Habenaria, 58, 60-61

Hackelia, 101

HALORAGACEAE, see

HALORAGIDACEAE

HALORAGIDACEAE, 93-94

Helianthus, 116

Heliopsis, 116

Hemerocallis, 56

Hepatica, 71

Heracleum, 95

Herniaria, 68

Hesperis, 74

Hieracium, 19, 21, 116-117
Hierochloe, 40

HIPPURIDACEAE, 94

Hippuris, 94

Holcus, 40

Humulus, 64

HYDROCHARITACEAE, 36

Hydrocotyle, 95

HYPERICACEAE, 88-89

Hypericum, 88-89, 89

Hystrix, 39

Ilex, 18,86

Impatiens, 18,87

IRIDACEAE, 58

Iris, 18, 58

ISOETACEAE, 26

Isoetes, 26

JUNCACEAE, 54-55

JUNCAGINACEAE, 36

Juncus, 15, 18, 54-55

Juniperus, 32

Kalmia, 17, 98

LABIATAE, see LAMIACEAE

Lactuca, 117-118

LAMIACEAE, 102-104

Laportea, 18, 64

Lappula, 101

Larix, 17, 31

Lathyrus, 83

Lechea, 89

Ledum, 98

Leersia, 40

Lemna, 18, 53

LEMNACEAE, 53

LENTIBULARIACEAE, 106-107

Leontodon, 118

Leonurus, 102

Lepidium, 15, 75

LILIACEAE, 55-57

Lilium, 56

Linaria, 104

Lindernia, 105

Linnaea, 14, 109

Liparis, 59

Listera, 59 
Lithospermum, 101

Littorella, 107

Lobelia, 10, 111

LOBELIACEAE, 111

Lolium, 40

Lonicera, 109

Lotus, 83

Ludwigia, 92

Luzula, 55

Lychnis, 68, 68

LYCOPODIACEAE, 25-26

Lycopodium, 25-26

Lycopus, 102

Lysimachia, 98-99

LYTHRACEAE, 91

Lythrum, 91

Maianthemum, 56

Malaxis, 12, 60

Malus, 80

Malva, 88

MALVACEAE, 88

Matricaria, 118

Matteuccia, 30

Medeola, 56

Medicago, 83-84

Melampyrum, 105

MELASTOMACEAE, 91

Melilotus, 84

Mentha, 102-103

Menyanthes, 17, 99

Milium, 40

Mimulus, 105

Mirabilis, 67

Miscanthus, 40

Mitchella, 14, 108

Mitella, 17, 76

Mollugo, 67

Monarda, 103

Moneses, 96

Monotropa, 96

MORACEAE, 64

Muhlenbergia, 40-41

Myosotis, 101

Myrica, 17, 63, 63

MYRICACEAE, 63

Myriophyllum, 18, 19, 93
NAJADACEAE, 35, see also POTAMOGETONACEAE

Najas, 35

Narcissus, 58

Nardus, 41

Nasturtium, 75

Naumbergia, 99

Nemopanthus, 18, 86

Nepeta, 103

Nuphar, 18, 69-70

NYCTAGINACEAE, 67

Nymphaea, 18, 70

NYMPHAEACEAE, 69-70

Nymphoides, 100

Oenothera, 93

OLEACEAE, 99

ONAGRACEAE, 92-93

Onoclea, 18, 30

OPHIGLOSSACEAE, 27

ORCHIDACEAE, 58-61

OROBANCHACEAE, 106

Orthilia, 97

Oryzopsis, 41

Osmorhiza, 95

Osmunda, 18, 27-28

OSMUNDACEAE, 27-28

Ostrya, 16, 64

OXALIDACEAE, 85

Oxalis, 85

Oxybaphus, 67

Panax, 94

Panicum, 41-42

PAPAVERACEAE, 73

Parthenocissus, 87

Pastinaca, 95

Petasites, 118

Phalaris, 42

Phegopteris, 30

Philadelphus, 76

Phleum, 42

Phlox, 100-101

Phragmites, 42

Physalis, 104

Physocarpus, 80

Picea, 17, 31

Pimpinella, 95 
PINACEAE, 31-32

Pinus, 16, 32

PLANTAGINACEAE, 107

Plantago, 15, 19, 107

Platanthera, 18, 60-61

Poa, 42-43

POACEAE, 36-44

Pogonia, 17, 61

POLEMONIACEAE, 100-101

Polygala, 85

POLYGALACEAE, 85

POLYGONACEAE, 65-66

Polygonatum, 56

Polygonum, 65-66

POLYPODIACEAE, 28-31

Polypodium, 30

Polystichum, 30

Pontederia, 18, 54

PONTEDERIACEAE, 54

Populus, 61-62

PORTULACACEAE, 67

Potamogeton, 18, 19, 33-35

POTAMOGETONACEAE, 20, 33-35

Potentilla, 80

Prenanthes, 118

PRIMULACEAE, 98-99

Proserpinaca, 94

Prunella, 103

Prunus, 80-81

Pteridium, 30

Puccinellia, 43

Pyrola, 18, 96-97

PYROLACEAE, 20, 96-97

Pyrus, 80

Quercus, 64

RANUNCULACEAE, 70-72

Ranunculus, 15, 71-72

Reseda, 75

RESEDACEAE, 75

RHAMNACEAE, 87

Rhamnus, 87

Rhexia, 91

Rhus, 86

Rhynchospora, 17, 52

Ribes, 18, 77

Robinia, 84
Rorippa, 75

Rosa, 81

ROSACEAE, 77-83

RUBIACEAE, 107-108

Rubus, 18, 81-82

Rudbeckia, 118

Rumex, 15, 66

Sagina, 68

Sagittaria, 10, 36

SALICACEAE, 61-63

Salix, 18, 62-63

Salsola, 67

Sambucus, 109

Sanguinaria, 73

Sanicula, 95

SANTALACEAE, 65

Saponaria, 68

Sarracenia, 17, 76

SARRACENIACEAE, 76

Satureja, 103

Saxifraga, 14, 77

SAXIFRAGACEAE, 76-77

Scheuchzeria, 36

Schizachne, 43

Scirpus, 18, 52-53

Scrophularia, 105

SCROPHULARIACEAE, 104- 106

Scutellaria, 103

Secale, 43

Sedum, 14, 76

Selaginella, 26

SELAGINELLACEAE, 26

Senecio, 119

Setaria, 43

Shepherdia, 91

Silene, 68-69

Sisymbrium, 75

Sisyrinchium, 58

Sium, 95

Smilacina, 17, 56-57

Smilax, 57

SOLANACEAE, 104

Solanum, 104

Solidago, 19, 119

Sonchus, 119-120

Sorbaria, 82

Sorbus, 82 
SPARGANIACEAE, 33

Sparganium, 18, 33

Spartina, 43

Spergula, 69

Spiraea, 82-83

Spiranthes, 61

Spirodela, 53

Stachys, 103

Steironema, 98

Stellaria, 69

Streptopus, 57

Subularia, 75

Symphoricarpos, 109

Symphytum, 101

Syringa, 99

Tanacetum, 120

Taraxacum, 120

TAXACEAE, 31

Taxus, 31

Teucrium, 104

Thalictrum, 72

Thelypteris, 30-31, 30

Thlaspi, 75

Thuja, 17, 32

THYMELAEACEAE, 91

Thymus, 104

Tiarella, 77

Tilia, 16, 88

TILIACEAE, 88

Tragopogon, 120

Triadenum, 89

Trientalis, 99

Trifolium, 84

Trillium, 57

Triosteum, 110

Triticum, 44
Tsuga, 16, 32

Tussilago, 120

Typha, 18, 32

TYPHACEAE, 32

ULMACEAE, 64

Ulmus, 64

UMBELLIFERAE, see APIACEAE

Urtica, 65

URT.ICACEAE, 64-65

Utricularia, 19, 106-107

Uvularia, 57

Vaccinium, 17, 98

Valeriana, 110

VALERIANACEAE, 110

Vallisneria, 19, 36

Verbascum, 105

Verbena, 102

VERBENACEAE, 102

Veronica, 105-106

Viburnum, 18, 110

Vicia, 84-85

Vinca, 100

Viola, 10, 18, 89-91

VIOLACEAE, 89-91

VITACEAE, 87

Waldsteinia, 83

Woodsia, 31

Woodwardia, 31

XYRIDACEAE, 53-54

Xyris, 53-54

ZOSTERACEAE, see

POTAMOGETONACEAE 




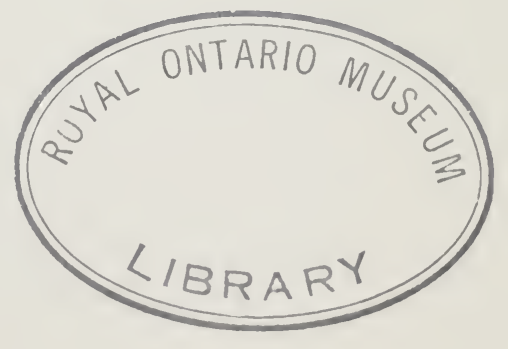


Further reading from the Royal Ontario Museum for botanists, naturalists, and gardeners:

\section{Shrubs of Ontario}

James H. Soper and

Margaret L. Heimburger

(Life Sciences Miscellaneous Publications)

- A comprehensive guide to shrubs in Ontario

- Includes more than 200 species

- Each accompanied by a full-page illustration and a map showing distribution throughout the province

"The work will stand for many years, probably decades, as the definitive statement on the subject."-R. F. Sutton, The Forestry Chronicle

\$32.95, Paper, 528 Pages, ISBN 0-88854-283-6

\section{Plant Alert}

\section{Deborah A. Metsger}

- A guide to poisonous and nonpoisonous plants commonly ingested in Ontario, Quebec, and adjacent areas of the United States

- 33 plants included, most illustrated in either full colour or black and white

- Includes wild plants, garden plants, and houseplants

- Text in both English and French

\$4.95, Paper, 56 Pages, ISBN 0-88854-348-4

Publication Services, Royal Ontario Museum, 100 Queen's Park, Toronto, Ontario, Canada, M5S 2C6, (416) 586-5581, Fax (416) 586-5863

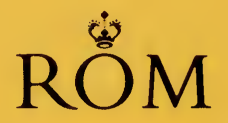

ROYAL ONTARIO MUSEUM LIFE SCIENCES

MISCELLANEOUS PUBLICATIONS

ISBN 0-88854-353-0

ISSN 0082-5093 